\title{
EFFECTS OF CORRUGATIONS ON STIFFNESS PROPERTIES OF COMPOSITE BEAMS FOR STRUCTURAL APPLICATIONS
}

\author{
A Thesis Presented to \\ The Faculty of California Poytechnic State University, \\ San Luis Obispo
}

\author{
In Partial Fulfillment \\ Of the Requirements for the Degree \\ Masters of Science in Aerospace Engineering
}

By

Jane Xiao

April 2019 
(c) 2019

Jane Xiao

ALL RIGHTS RESERVED 
COMMITTEE MEMBERSHIP

TITLE: $\begin{aligned} & \text { Effects of Corrugations on Stiffness Properties } \\ & \text { of Composite Beams for Structural Applications }\end{aligned}$

AUTHOR: Jane Xiao

DATE SUBMITTED: April 2019

COMMITTEE CHAIR: $\begin{aligned} & \text { Faysal Kolkailah, Ph.D, P.E } \\ & \text { Professor of Aerospace Engineering }\end{aligned}$

$\begin{array}{ll}\text { COMMITTEE MEMBER: } & \begin{array}{l}\text { Eltahry Elghandour, Ph.D } \\ \text { Professor of Mechanical Engineering }\end{array}\end{array}$

COMMITTEE MEMBER: $\begin{aligned} & \text { Dianne DeTurris, Ph.D } \\ & \text { Professor of Aerospace Engineering }\end{aligned}$

$\begin{array}{ll}\text { COMmitTeE MeMBER: } & \text { Tom Mase, Ph.D } \\ \text { Professor of Mechanical Engineering }\end{array}$ 


\begin{abstract}
Effects of Corrugations on Stiffness Properties of Composite Beams for Structural Applications

Jane Xiao
\end{abstract}

Composites have high strength-to-weight ratios, which is particularly desired for applications with weight restrictions. Common composite materials such as carbon fiber reinforced plastic $(\mathrm{CF})$ and fiber glass reinforced plastic (FG) were used in this research. While composite materials possess high stiffness and strength properties, the stiffness of composite laminates may be maximized by changing the geometry. By adding corrugations, the flexural stiffness is increased in one direction compared to the stiffness of a flat part with the same amount of material. Thus, stiffness increases without a change in weight.

The primary goal of this research was to investigate the stiffness characteristics of corrugated composite laminates under tensile and flexural load. The chosen corrugation geometry for investigation was a trapezoid. To observe the effects of corrugations, both flat and corrugated coupons were tested experimentally with the same procedures. Stiffness was calculated experimentally, analytically, and numerically in both directions. In this study, the longitudinal direction was defined as perpendicular to the corrugations and transverse direction was defined as the direction along the corrugations. The effects on stiffnesses of corrugated and flat composites were measured by comparing changes to the stiffness ratios in tension and bending. The stiffness ratio is the ratio of longitudinal stiffness to transverse stiffness.

The secondary aim of this research was to compare the corrugation effects on FG weave and cross-ply CF. This was interesting to observe the difference in corrugation effects on different composite materials. The FG laminates were manufac- 
tured from four plies of pre-impregnated Cytec MXB 7701/7781. The CF laminate consisted of five plies of pre-impregnated unidirectional Tencate TC250/M46J. The layup orientation of the $\mathrm{CF}$ laminate had alternating $0^{\circ}$ and $90^{\circ}$ plies, where the $0^{\circ}$ plies were in the transverse direction. Plies were directly laid on a flat plate and aluminum mold for flat and corrugated specimens, respectively. All flat and corrugated composites were cured in an autoclave under respective recommended cure cycles for each material. The tension and three-point bend tests were conducted on an Instron 8800 where the load was applied at a rate of 0.05 inches per minute.

The tensile ultimate load was the same between corrugated and flat specimens in the longitudinal direction. Meanwhile, the tensile ultimate load was greatly reduced for corrugated specimens in the transverse direction when compared to the flat specimens. Thus, corrugations had a larger impact in the transverse direction under tensile load for both materials.

By corrugating the composite layups, the ratio of stiffness in the longitudinal to extensional direction increases. For FG test coupons, the extensional stiffness ratio was increased from 1.0 to 49.3 due to corrugations. The flexural stiffness ratio was increased from 0.3 to 187.1 in corrugated FG coupons. For CF test coupons, the extensional stiffness ratio increased from 0.7 to 61.3. The flexural stiffness ratio of CF test coupons increased from 0.3 to 81.4. Corrugations had a greater effect on the cross-ply CF for both extensional and flexural stiffnesses. 


\section{ACKNOWLEDGMENTS}

I would like to thank my family for being supportive throughout my college career. This has been a long journey and it would not be possible with them.

I would like to thank members of my committee including Dr. Faysal Kohkailah, Dr. Elghandour, Dr. Tom Mase, and Dr. Diane Deturris for their time and support.

I would also like to thank my fellow graduate students who worked alongside me in the Composites Lab. We spent late nights at the lab together and it was always great to bounce ideas off of them. Thank you for helping me transport the heavy rolls of composite material to and from the freezer. This journey would not be the same without them.

I would like to extend my thanks to Cody Thompson, our lab technician, and his assistants for their help on manufacturing my mold. Also, thank you to Sam Moss for 3D printing a part for me. 


\section{TABLE OF CONTENTS}

Page

LIST OF TABLES . . . . . . . . . . . . . . . . . . ix

LIST OF FIGURES . . . . . . . . . . . . . . . . . xiv CHAPTER

1. INTRODUCTION ...................... 1

1.1 Composites Overview . . . . . . . . . . . . . . . . . . . 1

1.1.1 Introduction to Composites . . . . . . . . . . . . 1

1.1 .2 Types of Materials . . . . . . . . . . . . . . . 2

1.1.3 Manufacturing . . . . . . . . . . . . . . . 3

1.2 Corrugated Structures Overview . . . . . . . . . . . . . 4

1.3 Applications for Corrugated Composites . . . . . . . . . . 5

1.4 Previous Work . . . . . . . . . . . . . . . . . 6

1.5 Main Objective and Scope of Study . . . . . . . . . . . . 7

2. DESIGN OF CORRUGATION GEOMETRY . . . . . . . . . . 9

2.1 Design Factors $\ldots \ldots \ldots \ldots$

2.2 Initial Prototype $\ldots \ldots \ldots \ldots \ldots \ldots$

2.3 Final Design . . . . . . . . . . . . . . . . . . . . . . . 11

2.4 Mold Design . . . . . . . . . . . . . . . . . . . . . . . . 12

3. MANUFACTURING . . . . . . . . . . . . . . . . . 14

3.1 Mold Manufacturing . . . . . . . . . . . . . . . . . . . . 14

3.1 .1 Foam Mold . . . . . . . . . . . . . . . . . . . . . . . . . 14

3.1 .2 Final Aluminum Mold . . . . . . . . . . . . . . . . 16

3.2 Coupon Manufacturing . . . . . . . . . . . . . . . . . . 18

3.2.1 Material Properties Test Coupons . . . . . . . . . . . . 18

3.2 .2 Flat Test Coupons . . . . . . . . . . . . . . 20 
3.2.3 Corrugated Test coupons . . . . . . . . . . . . . . . . . . 21

3.3 Composite Material Cure Cycles . . . . . . . . . . . . . . . 25

3.3 .1 Fiberglass Cure Cycle . . . . . . . . . . . . 25

3.3.2 Carbon Fiber Cure Cycle . . . . . . . . . . . . . . 26

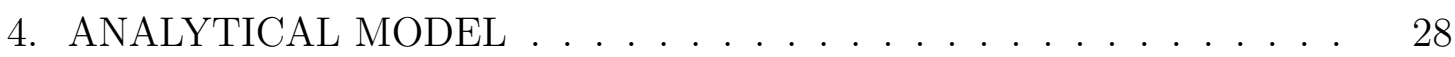

4.1 Mechanics of Composite Laminates . . . . . . . . . . . 28

4.2 Bernoulli-Euler Beam Method . . . . . . . . . . . . . . . 31

4.3 Xia and Friswell's Equivalent Model . . . . . . . . . . . . . . 33

5. FINITE ELEMENT ANALYSIS . . . . . . . . . . . . . . . . . 35

5.1 FEA Tensile Test Model . . . . . . . . . . . . . . . . . . 35

5.1 .1 Tensile Specimen: Geometry . . . . . . . . . . . . . 35

5.1.2 Tensile Specimen: Material and Section Assignment . . . . 35

5.1.3 Tensile Specimen: Assembly and Step . . . . . . . . . . 36

5.1.4 Tensile Specimen: Boundary Conditions and Loads _. . . 37

5.1 .5 Tensile Specimen: Mesh _. . . . . . . . . . . . . . 37

5.1.6 Tensile Specimen: Data Analysis _. . . . . . . . . . . . 39

5.2 FEA Flexure Test Model . . . . . . . . . . . . . . . . . . . 43

5.2.1 Flexure Specimen: Geometry _. . . . . . . . . . . . 43

5.2.2 Flexure Specimen: Material and Section Assignment _. . 43

5.2.3 Flexure Specimen: Assembly and Step . . . . . . . . . 44

5.2.4 Flexure Specimen: Boundary Conditions, Loads, and Inter-

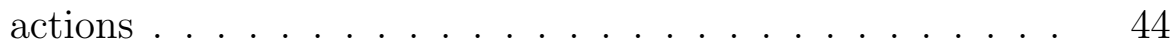

5.2 .5 Flexure Specimen: Mesh . . . . . . . . . . . . . . . 46

5.2.6 Flexure Specimen: Data Analysis _ . . . . . . . . . . 48

6. EXPERIMENTAL SET-UP AND TESTING . . . . . . . . . 50

6.1 Material Properties Test Procedures . . . . . . . . . . . . . 50

6.1 .1 Tensile Test . . . . . . . . . . . . . . . . . . 51 
6.1.2 Compression Test ................ . . 52

6.1.3 Poisson's Ratio . . . . . . . . . . . . . . . 52

6.1.4 Strain Gage Application . . . . . . . . . . . . . . 54

6.1.5 Volume Fraction ............. 56

6.2 Flat and Corrugated Specimen Test Procedure . . . . . . . . . . 57

6.2.1 Uniaxial Tension Test . . . . . . . . . . . 58

6.2.2 Three-Point Bend Test . . . . . . . . . . . . . 59

7. EXPERIMENTAL RESULTS AND DISCUSSION . . . . . . . . . . 61

7.1 Fiberglass Weave Mechanical Properties . . . . . . . . . . 61

7.2 Experimental Results . . . . . . . . . . . . . . 64

7.2.1 Extensional Stiffness Results . . . . . . . . . . . 65

7.2.2 Flexural Stiffness Results . . . . . . . . . . . 69

7.3 Experimental, Numerical, and Analytical Results and Discussion . 73

7.3.1 Comparison of Flat Specimen Stiffness . . . . . . . . . 73

7.3.2 Comparison of Corrugated Specimen Stiffness . . . . . . 75

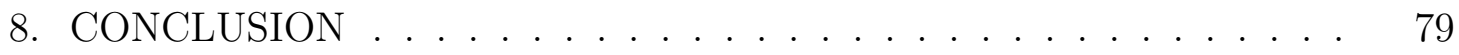

8.1 Future Recommendations . . . . . . . . . . . 80

BIBLIOGRAPHY .......................... 81

APPENDICES

A. FEA Material Properties . . . . . . . . . . . . . . . 83

B. Load-Deformation Curves . . . . . . . . . . . . . . . . . . . 84

C. Mesh Convergence . . . . . . . . . . . . . . . 88

D. MATLAB Code . . . . . . . . . . . . . . . . 92 


\section{LIST OF TABLES}

Table

Page

3.1 Foam Mold Materials . . . . . . . . . . . . . . . . . . . 16

3.2 Dimensions of Material Properties Test Coupons . . . . . . . . . . 19

3.3 Layup Orientation of Composite Parts . . . . . . . . . . . . . 20

3.4 Dimensions of Flat Test Coupons . . . . . . . . . . . . . . . . . . 21

3.5 Dimensions of Corrugated Test Coupons . . . . . . . . . . . . 24

4.1 Stiffness Results from Bernoulli-Euler Beam Method . . . . . . . 32

4.2 Stiffness Results from Friswell Method . . . . . . . . . . . . . . . 34

5.1 Dimensions of Flat FEA Models . . . . . . . . . . . . . . . . 36

5.2 Dimensions of Corrugated FEA Models . . . . . . . . . . . . 37

6.1 Types of Experimental Tests . . . . . . . . . . . . . 50

7.1 Cytec MXB 7701/7781 Material Properties . . . . . . . . . . . 63

7.2 Tensile Properties of Flat Test Specimens . . . . . . . . . . . . . 67

7.3 Tensile Properties of Corrugated Test Specimens . . . . . . . . . . 69

7.4 Flexural Properties of Flat Test Specimens . . . . . . . . . . 72

7.5 Flexural Properties of Corrugated Test Specimens . . . . . . . . . 72

7.6 Comparison of Extensional Stiffness of Experimental, Analytical, and FEA Flat Test Specimens . . . . . . . . . . . . . . 74

7.7 Percentage Difference of Extensional Stiffness Between Experimental, Analytical and FEA Flat Test Specimens . . . . . . . . . . 74

7.8 Comparison of Experimental, Analytical, and FEA Flexural Stiffness of Flat Test Specimens . . . . . . . . . . . . . 
7.9 Percentage Difference of Flexural Stiffness Between Experimental, Analytical and FEA Flat Test Specimens . . . . . . . . . 76

7.10 Comparison of Extensional Stiffness of Experimental, Analytical, and FEA Corrugated Test Specimens . . . . . . . . . 76

7.11 Percentage Difference of Extensional Stiffness Between Experimental, Analytical and FEA Corrugated Test Specimens . . . . . . . . 77

7.12 Comparison of Flexural Stiffness of Experimental, Analytical, and FEA Corrugated Test Specimens _. . . . . . . . . . 77

7.13 Percentage Difference of Flexural Stiffness Between Experimental, Analytical, and FEA Corrugated Test Specimens . . . . . . . 78

7.14 Extensional Stiffness Ratios Determined Experimentally _. . . 78

7.15 Flexural Stiffness Ratios Determined Experimentally . . . . . 78

A.1 Material Properties for FEA Models . . . . . . . . . . . . 83 


\section{LIST OF FIGURES}

Figure

Page

1.1 Prototype Trapezoidal Specimens with Aluminum Tabs . . . . . . 3

2.1 Initial Prototype Molds . . . . . . . . . . . . . . . . . . . . 10

2.2 Prototype Trapezoidal Specimens with Aluminum Tabs . . . . . . 11

2.3 Technical Drawing of the Final Design of Corrugated Specimen (units in inches) ....................... 12

2.4 Technical Drawing of the Aluminum Mold . . . . . . . . . . . . . 13

3.1 Foam Mold Covered by Tape . . . . . . . . . . . . . . . 15

3.2 Composite Mold Made with FG Bundles . . . . . . . . . . . . . . 16

3.3 Result of Foam Mold Warping Under Autoclave Pressure . . . . . 17

3.4 Aluminum Mold . . . . . . . . . . . . . . . . . . . . . 17

3.5 Material Test Coupons with Aluminum Tabs . . . . . . . . . 20

3.6 Comparison of the Effect of Using a Porous Sheet . . . . . . . . . 22

3.7 Manufacturing Assembly of Composite Layup . . . . . . . . . . . 22

3.8 Sealed Vaccuum Bag with Corrugated Layup Under Pressure . . . 23

3.9 Corrugated Coupons with Aluminum Tabs . . . . . . . . . . 25

3.10 Cytec MXB 7701 Cure Cycle . . . . . . . . . . . . . 26

3.11 Tencate TC250 Cure Cycle . . . . . . . . . . . . . . 27

4.1 Trapezoidal Unit Geometry for Golzar Method[6] . . . . . . . . . 31

4.2 Trapezoidal Unit Geometry for Equivalent Models [9] . . . . . . . 33

5.1 Cross Section of Tensile Corrugated FG FEA Model . . . . . . . . 36

5.2 Material Orientation of Corrugated FEA Model . . . . . . . . . . 38

5.3 Boundary Conditions on Corrugated Tensile FEA Model . . . . . 39 
5.4 Mesh Density of Corrugated Tensile FEA Model . . . . . . . . . . 40

5.5 Central Element Selection for Data Output . . . . . . . . . 41

5.6 FEA Deformation of Flat Tensile CF Specimens . . . . . . . . . . 41

5.7 FEA Deformation of Corrugated Tensile CF Specimens . . . . . . 42

5.8 Finite Element Flexure Model Assembly . . . . . . . . . . . . . . 45

5.9 Boundary Conditions on Corrugated Flexure FEA Model . . . . . 46

5.10 Mesh Density in Different Sections of Flexure FEA Model . . . . 47

5.11 Pin Meshed with Wedge and Hexagonal Elements . . . . . . . . . 47

5.12 FEA Deformation of Corrugated CF Specimens Under Flexural Load 49

6.1 FG Specimen in Compression Test . . . . . . . . . . . . . 52

6.2 Poisson's Ratio for a Rectangular Section . . . . . . . . . . . . 53

6.3 Computer Set-Up with Data Acquisition Unit and Instron . . . . 54

6.4 Test Specimens with Strain Gages Bonded . . . . . . . . . . . . 55

6.5 Strain Gage Bonded and Soldered to Wires . . . . . . . . . . . 56

6.6 FG Material Burned in Oven for Volume Fraction Test . . . . . . 57

6.7 Extensometer Secured to a Test Specimen . . . . . . . . . 58

6.8 Corrugated CF Specimen Aligned for Three-Point Bend Test . . . 59

6.9 Specimen Slipping in Three-Point Bend Test . . . . . . . . . . . 60

7.1 FG Material Test Specimen Measured with an Extensometer . . . 62

7.2 Stress-Strain Curves of FG Material Test Specimens . . . . . . . . 62

7.3 Pre- and Post-burn of FG Material for Volume Fraction Test . . . 64

7.4 Stress-Strain Curves of Flat Tensile Specimens . . . . . . . . . . . 66

7.5 Stress-Strain Curves of Corrugated Tensile Specimens . . . . . . . 68

7.6 Stress-Strain Curves of Flat Flexure Specimens _ . . . . . . . . . 71

7.7 Stress-Strain Curves of Corrugated Flexure Specimens . . . . . . 73

B.1 Load-Deformation Curves of Flat Tensile Specimens . . . . . . . . 84 
B.2 Load-Deformation Curves of Corrugated Tensile Specimens . . . . 85

B.3 Load-Deformation Curves of Flat Flexure Specimens . . . . . . . 86

B.4 Load-Deformation Curves of Corrugated Flexure Specimens . . . 87

C.1 Mesh Convergence Studies for Flat Tensile Models . . . . . . . . . 88

C.2 Mesh Convergence Studies for Flat Flexure Models . . . . . . . . 89

C.3 Mesh Convergence Studies for Corrugated Tensile Models . . . . . 90

C.4 Mesh Convergence Studies for Corrugated Flexure Models . . . . 91 


\section{CHAPTER 1: INTRODUCTION}

This chapter covers the background information about composite materials and corrugated structures. The background on composites include common materials it is composed of as well as the manufacturing methods used to make composites. Real world applications for corrugated composites are discussed. Previous work and research on corrugated composites are mentioned. Lastly, the goals of this research will be briefly summarized.

\subsection{Composites Overview}

Composite materials are increasingly used because of the high strength to weight ratio. Products created from composites can be found everywhere on a daily basis. This section covers the background information about composite materials including its definition, common types of composites, and manufacturing methods.

\subsubsection{Introduction to Composites}

Composite materials consist of two or more different materials whose combination possesses different structural properties from the individual materials. The materials combined to form composites are separated into two different types: matrix and fiber. Fibers are the main components that carry load. Meanwhile, the matrix holds the fibers together and effectively transfers the load to the fibers. The fiber or matrix alone would not be able to carry as much load as the composite formed by the combination.

Composite materials are known to be strong and lightweight. For metals, the

ultimate strength of the material occurs in the plastic region of the stress-strain curve. For some composites such as those utilized in this research, the ultimate strength occurs at the end of the linear elastic region because there is no plastic 
region for brittle materials. Common types of failure that occur in composites are delamination, bearing, and buckling. Delamination happens when plies in the composite layup separate. Brittle composites tend to have high energy (explosive) fractures when the material buckles. The common types of materials used to manufacture composites will be discussed in the following section.

\subsubsection{Types of Materials}

Different types of materials are combined to create composites. Common composites are carbon fiber reinforced plastic (CF), glass fiber reinforced plastic (FG), graphite, Nomex honeycomb, etc. The most common fibers used in composites are carbon and glass fibers in aerospace applications.

The matrix in a composite is important to reinforce the fibers. Common matrices include but are not limited to plastics, metals, and ceramics. The most common matrix materials are plastics, which is split into two categories. Thermoset polymers are resins where once it is set into the solid state, it cannot be melted and returned to the liquid state. However, the solid state of thermoplastics can be reversed under applied heat.

The structure of the fibers can be woven or unidirectional. Unidirectional fiber composites are just as they sound; the fibers are aligned in one direction. The fibers can be woven in different patterns which affect the mechanical properties, surface smoothness, and drape.

Surface smoothness is affected by the crimp in the fibers. The crimp of the fibers is caused by weaving the fibers over and under other fibers. Thus, the more the fiber passes over and under other fibers, the lower the surface smoothness. At the same time, more crimp results in slightly lower mechanical properties. Drape of the weave is the ability for it to conform and take the shape of the surface.

Different weave types favor different properties and the most common types 
are shown in Figure 1.1. The most common is the plain weave where each warp fiber goes over and under each weft fiber. The plain weave has a higher level of crimp and lower drape than all other types. Another popular weave is twill, which comes in $2 \times 2,3 \times 3,4 \times 4$, etc. Using the $2 \times 2$ twill as an example, the warp fibers go over two weft fibers before going under two weft fibers. At the same time, the weft fibers go over two warp fibers before going under two warp fibers.
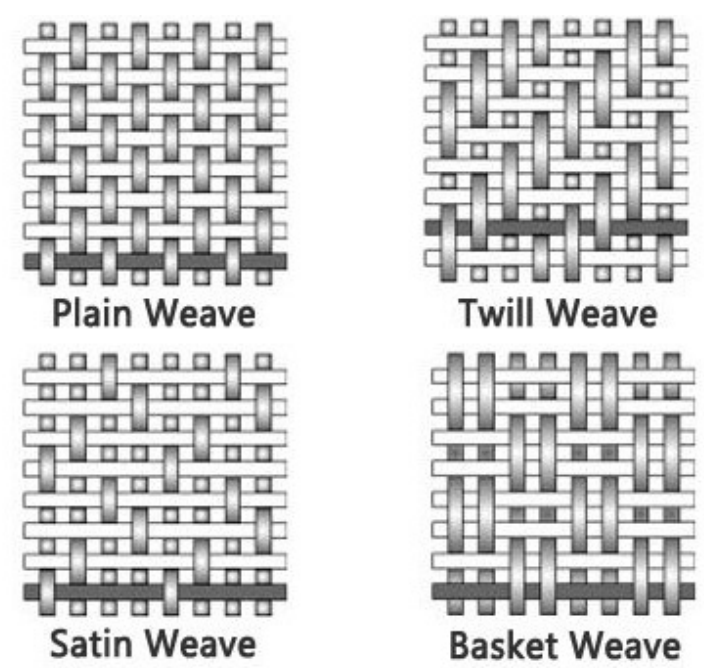

Figure 1.1: Prototype Trapezoidal Specimens with Aluminum Tabs

\subsubsection{Manufacturing}

There are two common methods of manufacturing composites: wet- and drylayups. The method is based upon the selected matrix material which will be epoxy resin for the scope of this research. If the composite is manufactured with dry fibers, a wet-layup will be required to integrate the resin matrix.

In a wet-layup, the epoxy must be mixed using part hardener and resin. Exact measurements must be taken at the discretion of the manufacturer's directions. There is a limited "work time" which is the amount of time before the epoxy starts to set and can no longer be moved. The cure time is the amount of time it takes for the epoxy to fully cure and harden. During the work time, the epoxy must be 
added to the fibers such that it is fully integrated. Excess epoxy should be scraped away because excessive resin will lower the strength of the cured composite. The epoxies used for wet-layups generally cure at room temperature under pressure from a vacuum pump. Other techniques such as vacuum infusion may also be used in wet-layups but this technique is not within the scope of this work.

The dry-layup involves working with pre-impregnated composites which must be cured in an autoclave. Pre-impregnated composites also referred to as prepreg are fiber sheets that are already infused with epoxy. Pre-preg are stored in a freezer under conditions as specified by the manufacturer until needed. This allows for a longer work time compared to the wet-layup, which requires one to work quickly. Epoxies used in pre-preg composites cure at a higher temperature, which requires an autoclave. Another benefit of this method is the ability to control the consistency of the fiber to resin ratio because pre-preg is already infused with the desired amount of resin. This method requires sealing the composite parts in a vacuum. The part is then cured under pressure in the autoclave with the appropriate cycle as specified by the manufacturer.

\subsection{Corrugated Structures Overview}

Corrugated structures were first utilized in the 19th century. The first patent for corrugated structure was obtained by Henry Robinson Palmer in 1829 for corrugated iron [1]. Since then, the most popular corrugated structures are corrugated paper commonly known as cardboard. Cardboard is used for shipping and packaging products. Despite the low bending strength of paper, cardboard is capable of sustaining greater loads in its corrugated shape.

The corrugations added to a material increases its stiffness in one direction. The direction perpendicular to the corrugations will be referred to as the longitudinal direction. Meanwhile the direction along the corrugation will be referred 
to as the transverse direction. Corrugations increase stiffness in the longitudinal direction. A corrugated structure compared to a flat laminate can sustain higher loads despite having the same amount of material. The effect of the corrugations differs between different unit shapes of the corrugation. Common shapes for the corrugations are circle, rectangle, trapezoid, and triangle.

The increased stiffness from corrugations have potential benefits for lightweight corrugated composites. The next section discusses applications for corrugated composites.

\subsection{Applications for Corrugated Composites}

There are different applications in which corrugated composites can be incorporated. Current research delves into the possibility of manufacturing morphing wings from corrugated composites. For a morphing wing application, the desired wing structure should be flexible in the chordwise direction while maintaining high stiffness and strength in the spanwise direction. Corrugated composites are lightweight structures with the desired stiffness characteristics. Research papers that discuss this application will be introduced in the following section.

Composite sandwich structures are very lightweight and possess high flexural strength and stiffness. Composite sandwich plates typically consist of two facesheets and a core. For the application of sandwich plates, corrugated composites can be used as the facesheets or the core. Composite sandwich structures maximize the material properties of its components to create a strong lightweight structure.

The facesheets are thin laminates usually composed of a few plies of composite material with high stiffness to carry in-plane and flexural loads. Facesheets can be manufactured from any type of composite material such as carbon, glass, or

aramid fibers. The core is a lightweight and thick material bonded to a facesheet 
on either side. Popular core materials are Nomex or aluminum honeycomb or high density foam. The structural contribution of the core is to carry shear loads.

Corrugated composites can be used in the aerospace industry for airframe structures such as the fuselage or wing structures. Corrugated composites can also be implemented in ships, which already utilizes fiberglass composites for the hull. Corrugated composites have higher strength to weight ratio, which is a desirable characteristic for applications in many fields.

\subsection{Previous Work}

Past research papers are great references to provide background information on the current research topic. Researching previous efforts on the subject avoids reinventing the wheel and making the same mistakes, which costs time and money. Throughout this research, previous works were referenced for test sample geometries and analytical methods.

Research on corrugated carbon fiber structures was conducted by Tomohiro Yokozeki et al.[2] in 2006 for the application of wing structures. His research involved testing circular corrugated composites for tensile and flexural stiffness properties. The corrugated specimens were also tested with modifications to increase the stiffness ratio between the two directions. The corrugated carbon fiber composite was modified to add unidirectional carbon fiber tubes along the corrugations to increase the stiffness in the direction perpendicular to the corrugations. Christophe Thill et al. [3] studied trapezoidal corrugated composites under tensile load. His research was on the stiffness properties of aramid-epoxy laminates under tensile load in the longitudinal direction.

Corrugated composites have potential for implementation into different applications as mentioned in the previous section. Iman Dayyani et al.[4] researched elastomeric coated corrugated composites for the application of morphing wings. 
While the corrugated composite laminate was implemented as a core, Jack Reany and Joachim L. Grenestedt [5] implemented it as the skin or facesheet of a foam core sandwich panel.

Four different corrugation shapes were tested in research by Golzar and Ghabezi [6]. Fiberglass specimens were manufactured with different frequencies for each corrugation shape. The height of the corrugations and size of the coupons remained constant while the number of corrugation units varied. The analytical method used by Golzar was based on Euler-Bernoulli beam theory. This method was adopted for my research to compare the accuracy of predicting stiffnesses of corrugated structures.

The flexural rigidities of corrugated sheets were modeled by using an equivalent orthotropic plate as investigated by McFarland[7]. However, the model only predicted the flexural stiffnesses. An equivalent orthotropic model was developed by Samanta and Mukhopadhyay[8] to determine the extensional rigidity of trapezoidal corrugated sheets.

The second analytical method adopted in this research was created by Xia and Friswell in 2011[9]. They developed equivalent orthotropic models to predict both extensional and flexural stiffnesses of circular and trapezoidal corrugated laminates based on the geometry of its unit cell. This equivalent model was developed to provide a means of a simple model of corrugated laminates without assuming an isotropic material.

\subsection{Main Objective and Scope of Study}

The objective of this research is to determine the effects of corrugations on stiffness properties of composite beams for structural application. These properties vary depending on the direction, so the effects will be studied for both the longitudinal and transverse directions. 
Corrugated composites show great potential for implementation into different applications and industries. One goal of this research is to show the stiffness characteristics of corrugated composites, which are manufactured from different materials. In order to determine the extensional and flexural stiffnesses, test specimens will be manufactured from fiberglass weave and cross-ply carbon fiber, which were materials donated to the Aero Composites Lab. Corrugated FG laminates would be geared towards applications where the desired characteristics include lightweight and flexibility in one direction. Meanwhile, corrugated CF laminates are desired for applications where weight may be less of a concern because CF is denser than FG. However, CF laminates have higher strength and stiffness than FG laminates.

The corrugated FG and CF specimens cannot be compared side by side because they will be manufactured from very different materials. As a control, flat composite test coupons will be manufactured with the same amount of material. The ratio of longitudinal to transverse stiffness will be compared between the flat and corrugated specimens.

Design of the corrugation unit geometry and factors that contributed to the design will be elaborated upon in Chapter 2. The manufacturing process of the corrugated specimens is a significant part of this research. Consistent test specimens are necessary to obtain consistent experimental results. All the challenges and the refined manufacturing process will be described in Chapter 3. Chapter 4 will cover the analytical methods used to predict the stiffness properties of corrugated composites. The Finite Element Analysis will be discussed in Chapter 5. Experimental procedures for set-up and testing will be in Chapter 6. The experimental results and comparison with analytical and FEA results will be discussed in Chapter 7. Finally, Chapter 8 will summarize this research and provide recommendations for future studies. 


\section{CHAPTER 2: DESIGN OF CORRUGATION GEOMETRY}

This chapter will discuss factors that affect the design of the corrugation geometry. Furthermore, the initial designs were tested and results of the first prototype were used to finalize the design. The final design of the corrugation geometry will be shown in this chapter. A mold based on the final corrugation geometry was designed in order to manufacture the specimens.

\subsection{Design Factors}

The geometry of the corrugation unit was designed to be similar to reference geometries. Popular unit geometries include circular, sinusoidal, rectangular, and trapezoidal shapes. For the scope of this research, the geometry of the corrugation was kept constant for simplicity. After selecting the shape of the unit cell, the ratios in the geometry were kept constant and scaled. A design with larger corrugations would increase the ease of manufacturability but the size of the corrugation was limited by the height of the specimen. The maximum allowable height of the corrugation was 0.18 inches to fit in the Instron grips without special test fixtures. This height accounted for the thickness of the aluminum tabs, which were bonded to both sides of tensile coupons. The tensile test procedure was simplified with specimens that fit within the grips because no additional mounts or clamps were

necessary. See the Initial Prototype section below for more details about the geometry selection process.

\subsection{Initial Prototype}

For the prototype, I narrowed down the geometry selection to two popular shapes, which were manufactured and tested. The selected shapes were semi-circles and 
trapezoids. The rectangular unit was not chosen because manufacturing difficulties were anticipated for geometries with $90^{\circ}$ angles. Problems such as releasing the specimen from the mold and contouring the composite material to the sharp corners were considered. I decided to test the ease of manufacturability for the two shapes. For both geometries, the height of the corrugation was kept consistent. To create a quick mold to manufacture the prototype specimens, I decided to use a pink foam (FOAMular 150) for the mold. The foam molds were cut using the 2D CNC hot wire machine in the UAV lab on campus. The two molds are shown in Figure 2.1 below.

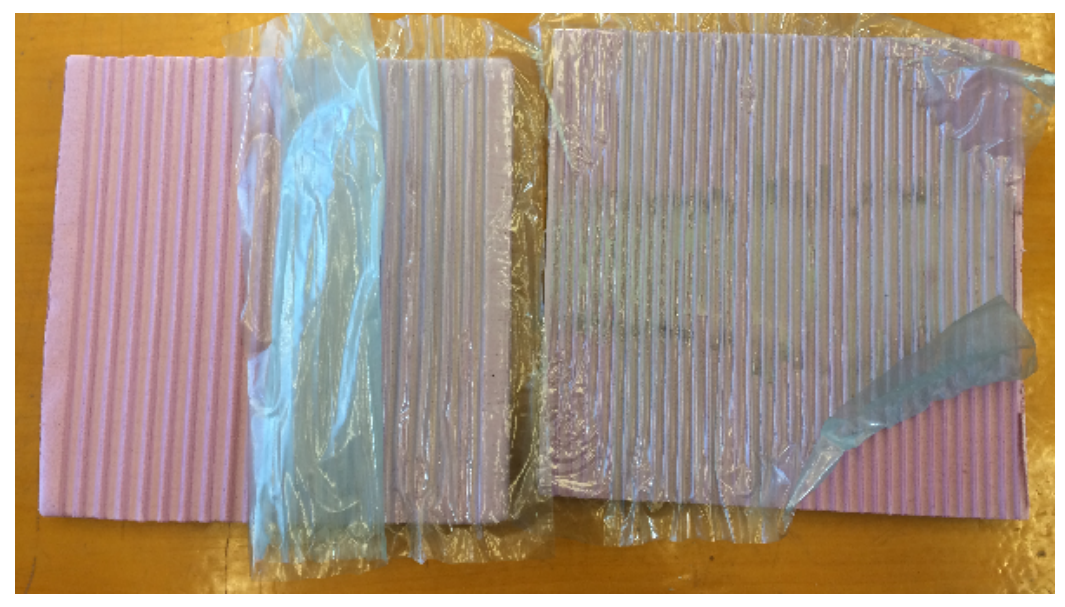

Figure 2.1: Initial Prototype Molds

This foam could not take the pressure or heat necessary to cure a pre-impregnated composite. The prototype specimens were created using a wet layup of a carbon fiber weave. To prepare the mold for a layup, I sprayed the molds lightly with adhesive and applied a thin vacuum bag film onto the surface. The vacuum bag film provided a layup surface for the composite. Spray release was used on the non-porous material to help release the composite from the mold. The main purpose of this prototype was to test the ease of the layup process. Thus, the material type and properties were not crucial in the test.

From the wet layup, the circular units proved to be more difficult to contour to 
the mold and failed to form the desired shape. The manufacturing process to create the desired shape would be even more difficult for pre-preg composites so the circular geometry was not a viable option. The trapezoidal prototype specimens were flawed but resembled the desired shape. Thus, only the trapezoidal plate was cut to 10 in. by 1 in. coupons and tested in the transverse direction in a uniaxial tension test. Aluminum tabs were bonded to the specimens with structural adhesive, which filled the gap between the corrugations. These specimens are shown in Figure 2.2.

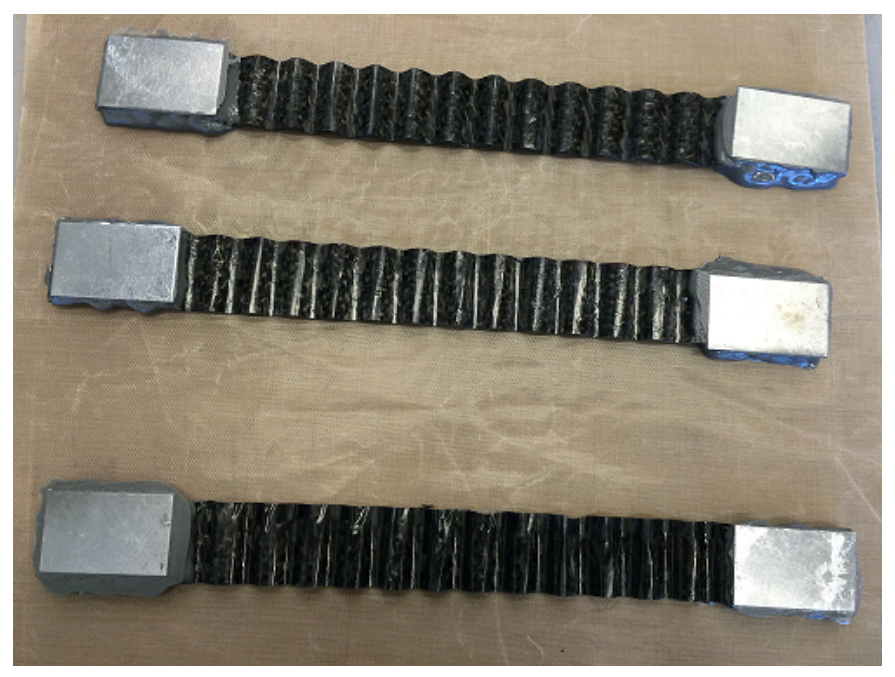

Figure 2.2: Prototype Trapezoidal Specimens with Aluminum Tabs

By testing the specimens, I was able to determine whether the aluminum tabs successfully distributed the pressure from the Instron grips. The specimens failed due to interlaminar delamination between the plies, which was an acceptable failure mode. Moving forward from the results of the prototypes, the trapezoidal unit was chosen and further changes in the design will be addressed in the next section.

\subsection{Final Design}

From the prototype test results, the trapezoidal unit geometry was chosen. The dimensions on the sides of the trapezoid were kept the same. However, upon 
consideration for the manufacturing process of the mold, a change in the angle geometry was made. In the prototype design, the angle shown in Figure 2.3 was $63.3^{\circ}$. This angle would be difficult and time consuming to manufacture without a specialized tool bit, which would not be cost-effective. For the final design, I modified the angle to $60^{\circ}$ because the geometry was very similar to the prototype design and the modification eliminated the need for a specialty tool bit. The final design of the corrugated specimen is shown in Figure 2.3. The mold necessary to create this geometry is discussed in the next section.

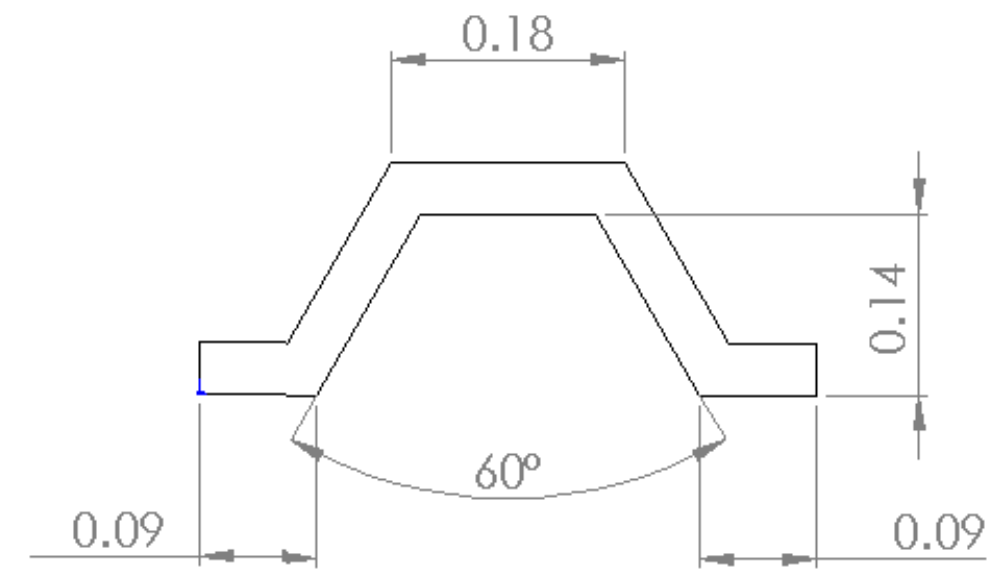

Figure 2.3: Technical Drawing of the Final Design of Corrugated Specimen (units in inches)

\subsection{Mold Design}

The geometry of the mold was designed to manufacture specimens with the desired geometry. The valleys and mountains in the mold had different dimensions as shown by Figure 2.4 in order to create symmetrical specimens. To maximize the number of specimens manufactured per cure cycle, the mold was designed to have a minimum length and width of 10 inches. The total length of the corrugated specimens was 10 inches in both directions. A corrugated plate could be cut to 
generate 6-7 specimens per layup. One layup could generate a 10 square inch corrugated plate. The final mold was machined out of a 12x12x1 inch block of aluminum 6061. See Section 3.1 for more details.
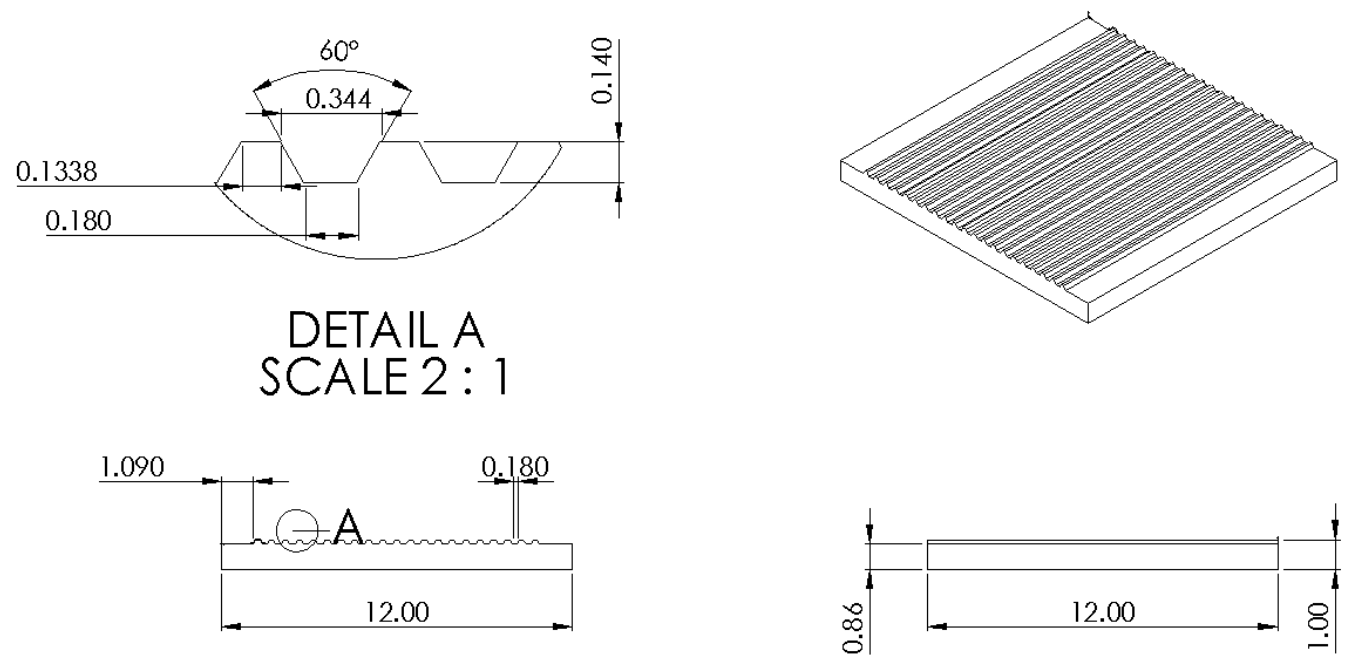

Figure 2.4: Technical Drawing of the Aluminum Mold 


\section{CHAPTER 3: MANUFACTURING}

\subsection{Mold Manufacturing}

The manufacturing process of two molds will be described. The foam mold was the initial design but was unsuccessful due to its material properties. The manufacturing process of the foam mold will be documented as it would have worked with an appropriate material. The second mold was manufactured out of aluminum and this was the mold used to manufacture test coupons for this research.

\subsubsection{Foam Mold}

Two molds, female and male, were created in order to create consistent and accurate geometries for the corrugated test coupons. Both molds must withstand the pressure and heat in the autoclave as specified for the material cure cycles. However, the desired properties were different for each mold. The female mold was designed to be rigid because the composite material must contour to its shape. Meanwhile, the male mold was designed to be very flexible in the corrugation direction to allow one corrugation unit to be formed at a time.

The female mold was machined out of a high-density foam found in the Aero Composites Lab. The part was machined using a CNC router and the RhinoCAM software in the Defabrication Lab at Cal Poly. Three different bits were used in the machining process. First, a half-inch end-mill was used to cut and even out the surface. Secondly, a one-eighth end-mill cut the slots for the trapezoids. And lastly, a special $60^{\circ}$ chamfer mill was used to cut out the sides of the trapezoids.

The male mold had to be almost identical to the female mold because the trapezoidal geometry of the corrugated coupons was symmetrical. First, I copied the features of the female mold by laying up on it, creating a secondary mold for 
the male mold. To create this secondary mold, I applied scotch packaging tape on the surfaces of the female mold. The foam mold sealed by clear tape is shown in Figure 3.1.

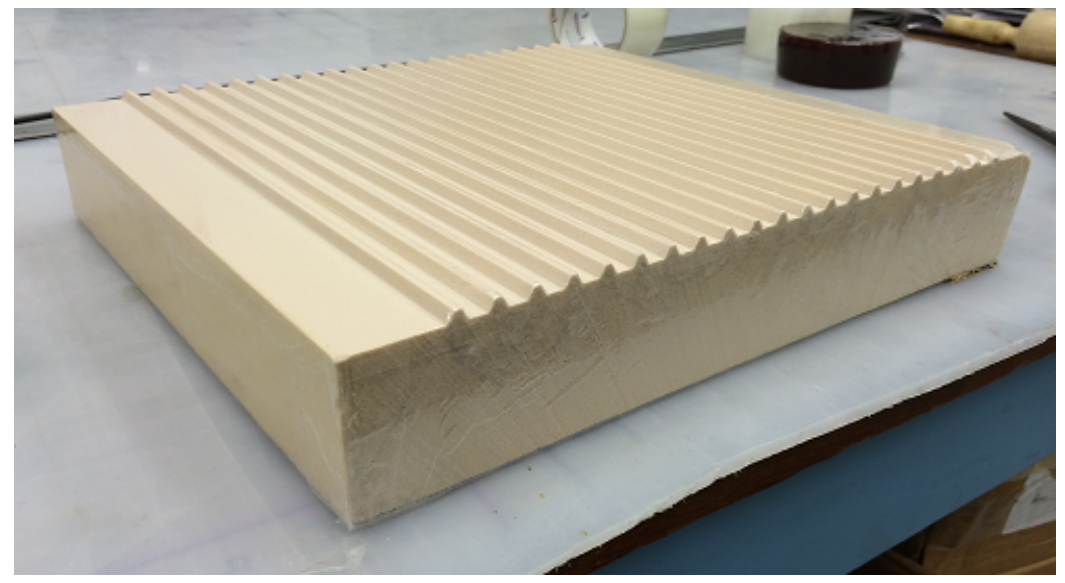

Figure 3.1: Foam Mold Covered by Tape

Because the female mold was made from foam, the tape had to be applied to create a release surface. Next, I applied a release agent (Chemlease 41-90 EZ) on the tape which allows any layup to be released easily. Then I lined the edges of the female mold with tape creating a barrier to prevent the resin from leaking. Lastly, the resin was poured in and left to cure until hardened. After taping the surfaces of the secondary mold, the release agent was applied for easier release from the mold.

The male mold was made flexible by using unidirectional glass fibers in the valleys of the mold and a thin carbon fiber weave on top as a connector. The list of materials used to manufacture the molds are summarized in Table 3.1. Glass fibers with a length of 14 inches were roughly measured out to fill the valleys of the secondary mold. The fibers were individually bundled using rubber bands on the two ends as shown by the composite mold in Figure 3.2. The epoxy was mixed and applied to the bundles of fiberglass. Each bundle must be pressed into the valley; otherwise, air pockets would form. I had to work quickly because the epoxy began to cure in about 30 minutes. Lastly, the 12 in. by 12 in. carbon fiber weave 
was laid on top to connect the fiberglass bundles together. The wet layup was then sealed in a vacuum bag using the vacuum table in the Composites lab.

Table 3.1: Foam Mold Materials

\begin{tabular}{|c|c|c|}
\hline Part & Material & Dimensions \\
\hline Female Mold & Foam & 12 in. by 12 in. by 2 in. \\
Male Mold Corrugations & Unidirectional FG & 14 inches long \\
Male Mold Plate & CF weave & 12 in. by 12 in. \\
\hline
\end{tabular}

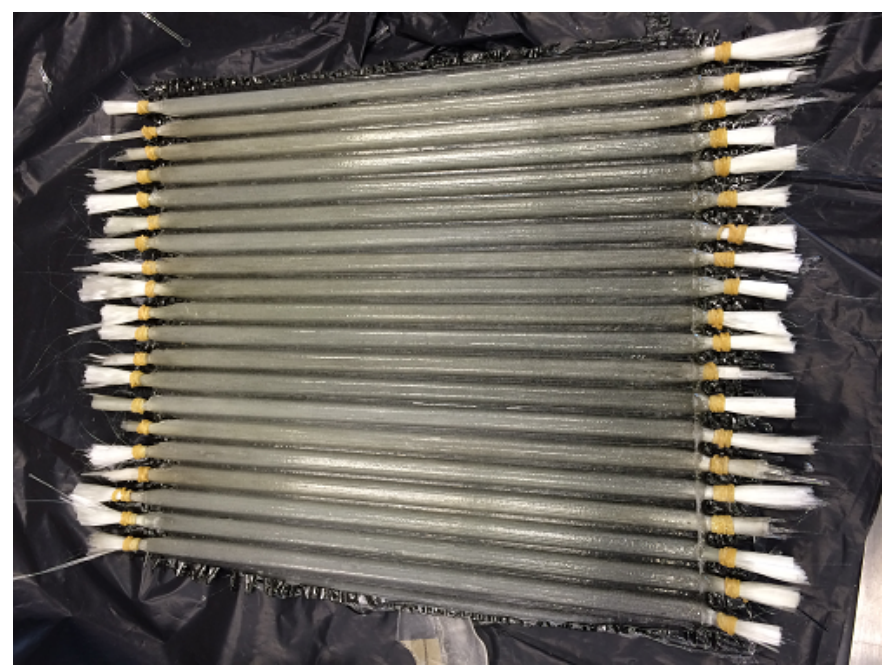

Figure 3.2: Composite Mold Made with FG Bundles

Unfortunately, upon curing fiberglass with the foam mold for the first time in the autoclave, the foam warped under the pressure of $45 \mathrm{psi}$. The warped mold is shown in Figure 3.3.

At this time, I realized that this foam was a different type of foam from what had been used by another graduate student. The material was what we had in the lab so I strongly advise testing a sample piece in the autoclave prior to machining the mold for future reference.

\subsubsection{Final Aluminum Mold}

A new mold had to be machined because the foam mold failed. I decided to make an aluminum mold instead to avoid a similar incident from reoccurring. The 


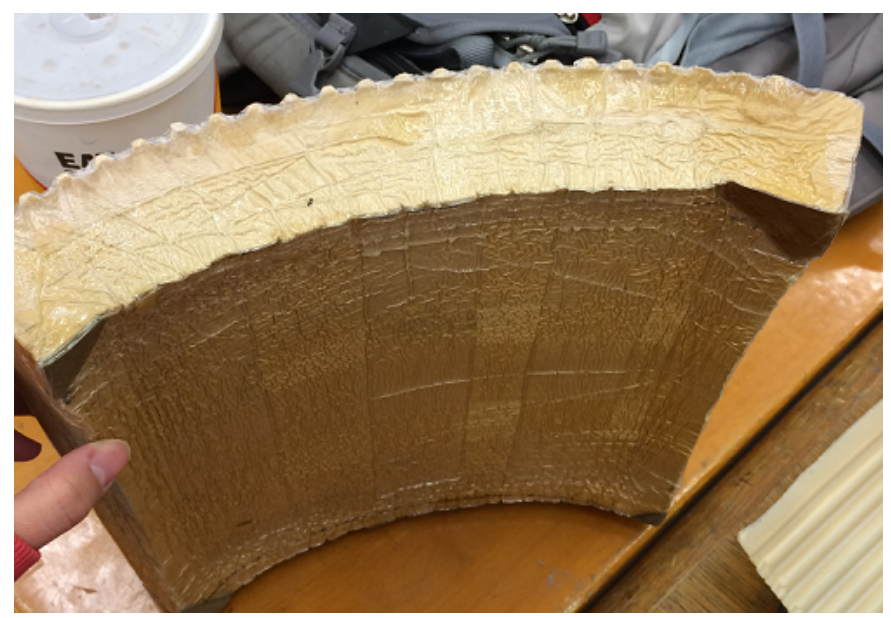

Figure 3.3: Result of Foam Mold Warping Under Autoclave Pressure

problem with the mold resulted in a big delay. The material chosen was Aluminum 6061, which is a general purpose aluminum. The mold was machined out of a 12 in. by $12 \mathrm{in.}$ by $1 \mathrm{in}$. aluminum plate in a similar fashion as the foam mold mentioned previously. This final mold is shown in Figure 3.4.

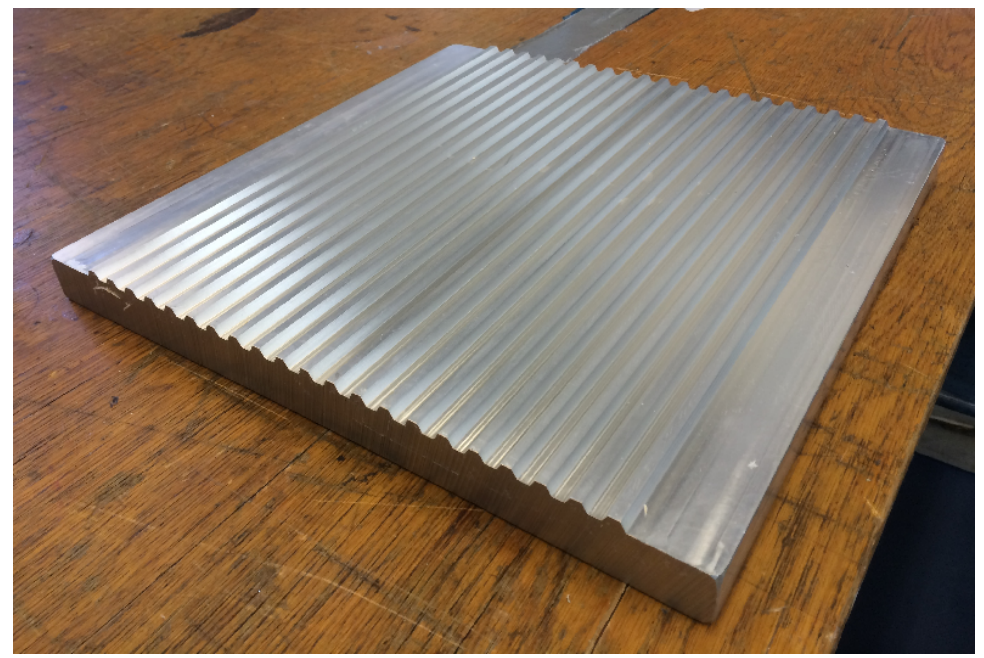

Figure 3.4: Aluminum Mold

The composite mold mentioned above was discarded and only the aluminum mold was used. The composite mold was ineffective unless the geometry matched perfectly with the layup. Otherwise, the pressure on the layup would be inconsistent in different regions. The Fiberglass (FG) and Carbon fiber (CF) materials had different thicknesses, so two additional molds would have been required. It was 
not cost-effective in regards to time spent waiting for additional molds to be machined. Thus, I decided to proceed with manufacturing the corrugated composites with a single mold.

The mold was prepared by thoroughly cleaning it with alcohol or acetone. Sealant was applied to the surfaces to seal any cracks or chips where resin could seep into. Next, Chemlease 41-90 EZ was applied per manufacturer instructions. This product helps release the composites from the mold post-cure.

\subsection{Coupon Manufacturing}

This section discusses the manufacturing process used to create the test coupons. The test coupons include material test coupons for the determination of its material properties, flat coupons, and corrugated coupons. The dimensions and layup procedures for each type of coupon are discussed along with any problems or difficulties.

\subsubsection{Material Properties Test Coupons}

Coupons for determining material properties follow ASTM standards for each corresponding test, which have size specifications. For uni-axial tension test coupons, ASTM D3039 specifies the dimensions based on fiber orientation. The fiberglass material test coupons follow the geometry recommendations of the balanced and symmetric orientation because it is a weave. For tensile coupons, 10 plies of fiberglass were laid up together for a thickness of 0.1 inches. The compression coupons follow ASTM D3410. Fiberglass compression coupons were composed of 7 layers for a thickness of 0.069 inches. The coupon dimensions are listed in Table 3.2.

The same carbon fiber material was used previously by other graduate students, so the material properties were taken from their results. The carbon fiber coupons had a width of 1 inch instead of the recommended 0.5 inches because strain gages 
Table 3.2: Dimensions of Material Properties Test Coupons

\begin{tabular}{|c|c|c|c|c|}
\hline Material & Test & Length[in] & Width [in] & Thickness [in] \\
\hline FG & Tension & 10.0 & 1.0 & 0.10 \\
FG & Compression & 4.75 & 1.0 & 0.069 \\
CF & Tension & 10.0 & 1.0 & 0.04 \\
CF & Compression & 4.50 & 1.0 & 0.07 \\
\hline
\end{tabular}

were applied and required a bond area greater than 0.5 inches. The actual coupon sizes are shown in Table 3.2.

The following layup procedure for these coupons will be applicable to a basic layup and will be used for the flat coupons in the next section. A non-porous sheet was taped to a flat aluminum plate. Composite material was laid on top of the non-porous sheet followed by another non-porous sheet. Breather material was then laid atop everything to uniformly distribute air flow during the vacuum process. Sealant tape was used between the aluminum plate and vacuum bag. Two vacuum connectors were used to connect the vacuum bag to the vacuum pump. The bottom part of the connectors must be inside the vacuum bag and on top of the breather prior to sealing it. While the vacuum pump was running, wrinkles were smoothed out from the area of the vacuum bag that was on top of the material. After checking the seal for any leaks, the plate was cured in the autoclave with the appropriate cure cycle for each material, which is specified in Section 3.3.

Post-cure of the layup, the edges of the plates were trimmed of the excess resin. The coupons were cut to size using a tile saw. After cleaning the coupons, aluminum tabs were glued to the ends of each coupon as shown in Figure 3.5. Aluminum tabs were adhered to the coupons to prevent slipping during testing. Excess adhesive coming out from beneath the tab ensures a good bond and distributes the stress caused by pressure from the Instron grips. Aluminum tabs were cut from plates with a thickness of 0.065 inches into 2 in. by 1 in. pieces. The alu- 
minum tabs were 2 inches long because that is the longest length that fits within the Instron grips.

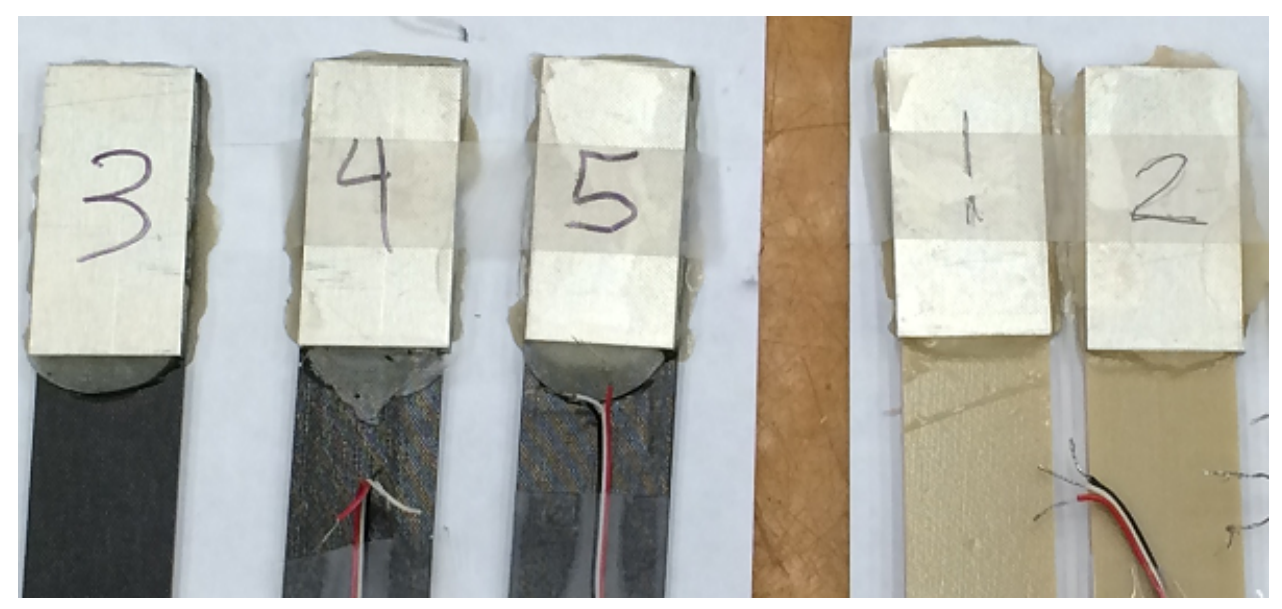

Figure 3.5: Material Test Coupons with Aluminum Tabs

\subsubsection{Flat Test Coupons}

Test coupons were composed of 4 or 5 layers of pre-preg composite material. See Table 3.3 for the layup sequence for each type of material. The material was cut into 14.5 in. by 10.5 in. rectangles with excess material on the edges which were trimmed post-cure. The layup procedure was the same as that for the material test coupons mentioned above.

Table 3.3: Layup Orientation of Composite Parts

\begin{tabular}{|c|c|}
\hline Material & Layup Sequence \\
\hline FG & {$[0]_{4}$} \\
CF & {$[0 / 90 / \overline{0}]_{s}$} \\
\hline
\end{tabular}

After the cure, the edges of the plates had to be trimmed and coupons were cut to size with a table saw. Edges were trimmed because excess resin pools around the edges due to the vacuum pressure during the cure cycle. The dimensions for the longitudinal and transverse coupons are shown in Table 3.4. While there was no ASTM standard for testing corrugated composites, these test coupon dimensions 
were based on ASTM 3039 and The lengths listed are the total lengths of the coupons including the two-inch-long aluminum tabs. For the first set of transverse flat coupons, I forgot to take into account that the area where tabs were applied is different between the flat and corrugated coupons. That resulted in flat transverse coupons with a longer gage length than desired. Dimensions of the flat coupons were designed such that the amount of material in the gage section was the same as the corrugated coupons. Continue to the next section for the manufacturing procedure for corrugated coupons.

Table 3.4: Dimensions of Flat Test Coupons

\begin{tabular}{|c|c|c|c|c|}
\hline Material & Direction & Length[in] & Width [in] & Thickness [in] \\
\hline FG & Longitudinal & 10.0 & 1.27 & 0.04 \\
FG & Transverse & 12.28 & 1.0 & 0.04 \\
CF & Longitudinal & 10.0 & 1.27 & 0.044 \\
CF & Transverse & 12.28 & 1.0 & 0.044 \\
\hline
\end{tabular}

\subsubsection{Corrugated Test coupons}

The layup procedure was slightly different for each material type. The layup sequences were the same as the flat coupons in Table 3.3. The general vacuum bag procedure was followed. First, the aluminum mold was coated with 2-3 layers of 41-EZ90 release to ensure the composite part separates from the mold. The layers in the vacuum bag resembled that of the flat plates mentioned in the previous section. An additional layer of porous material on top of the composite helped bleed out excess epoxy trapped in the corners as shown in Figure 3.6.

The composite material was applied directly on the aluminum followed by a porous sheet, a non-porous sheet, breather, and vacuum bag film. The layers within the vacuum bag are represented in Figure 3.7. 


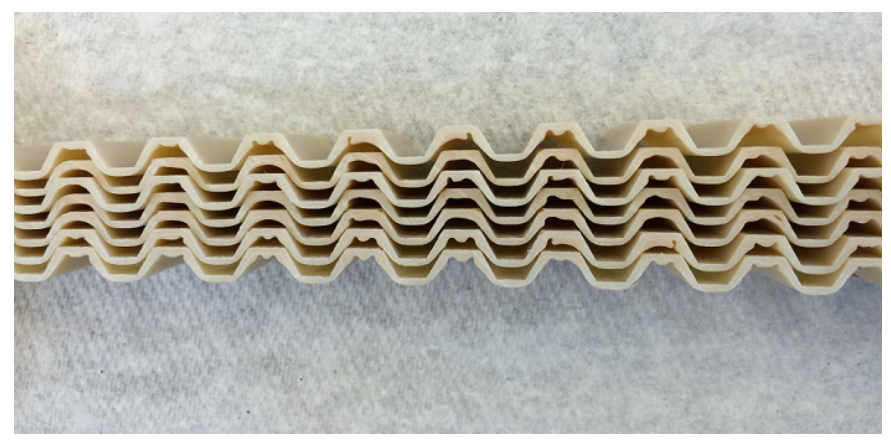

(a) Before

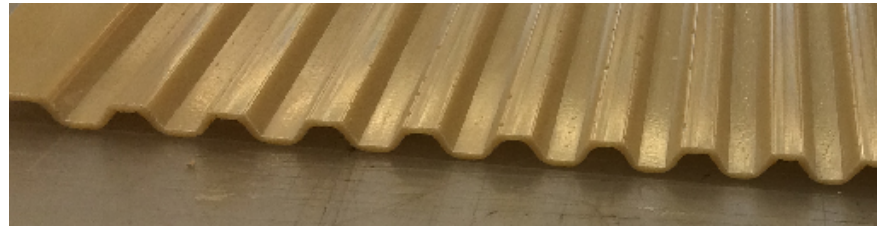

(b) After with Non-porous Sheet

Figure 3.6: Comparison of the Effect of Using a Porous Sheet

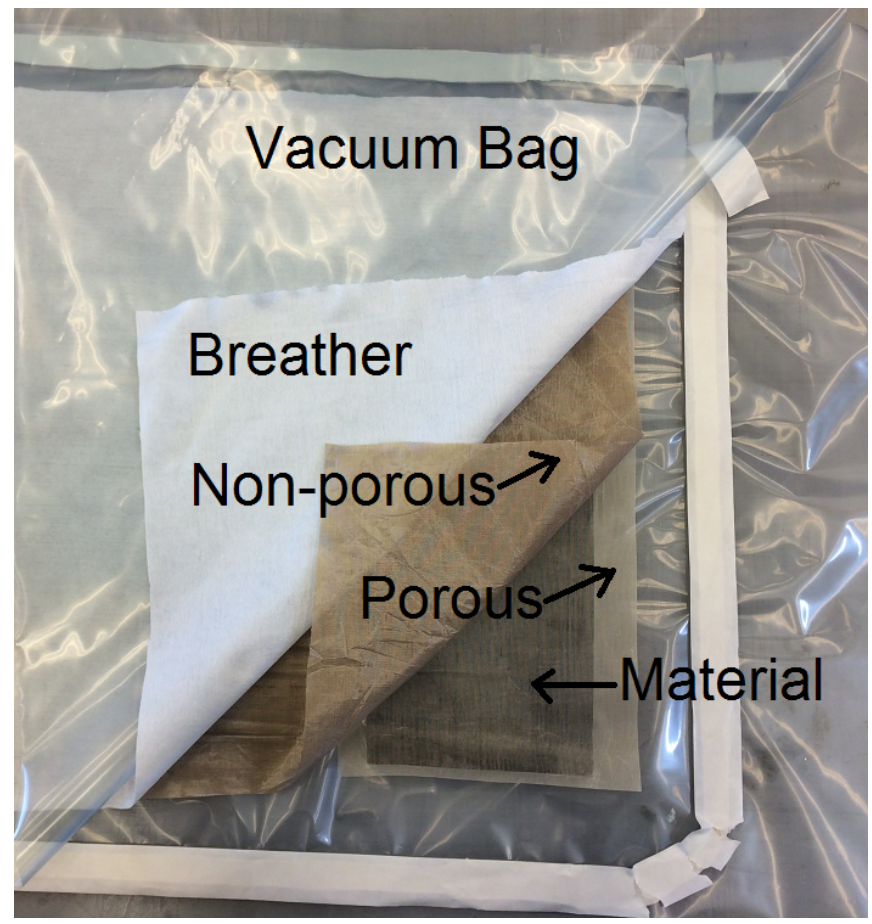

Figure 3.7: Manufacturing Assembly of Composite Layup 
For pure fiberglass coupons, four plies of the pre-preg material were laid on top of one another prior to its application to the male mold. I used the heat table in the Composites Lab to warm up the thick block of aluminum to $80-90^{\circ} \mathrm{F}$. This made the pre-preg tackier and helped it stay in the grooves of the mold. The material was pressed into the mold one trapezoid unit at a time using a flat plastic tool to prevent damage to the mold. This process took about 3 hours with a lot of strain on the hands. I had a small trapezoid piece 3D printed to assist in pressing the material into the grooves. Since this was a long process, the material wanted to relax out of the grooves. Thus, I had to constantly go back and re-apply pressure to keep it from moving. By working on one unit at a time, the material really contoured to the shape of the mold. The entire structure was sealed in a vacuum bag with sealant tape and air was pumped out as shown in Figure 3.8. Then the composite was cured in the autoclave according to fiberglass cure cycle in Section 3.3.1.

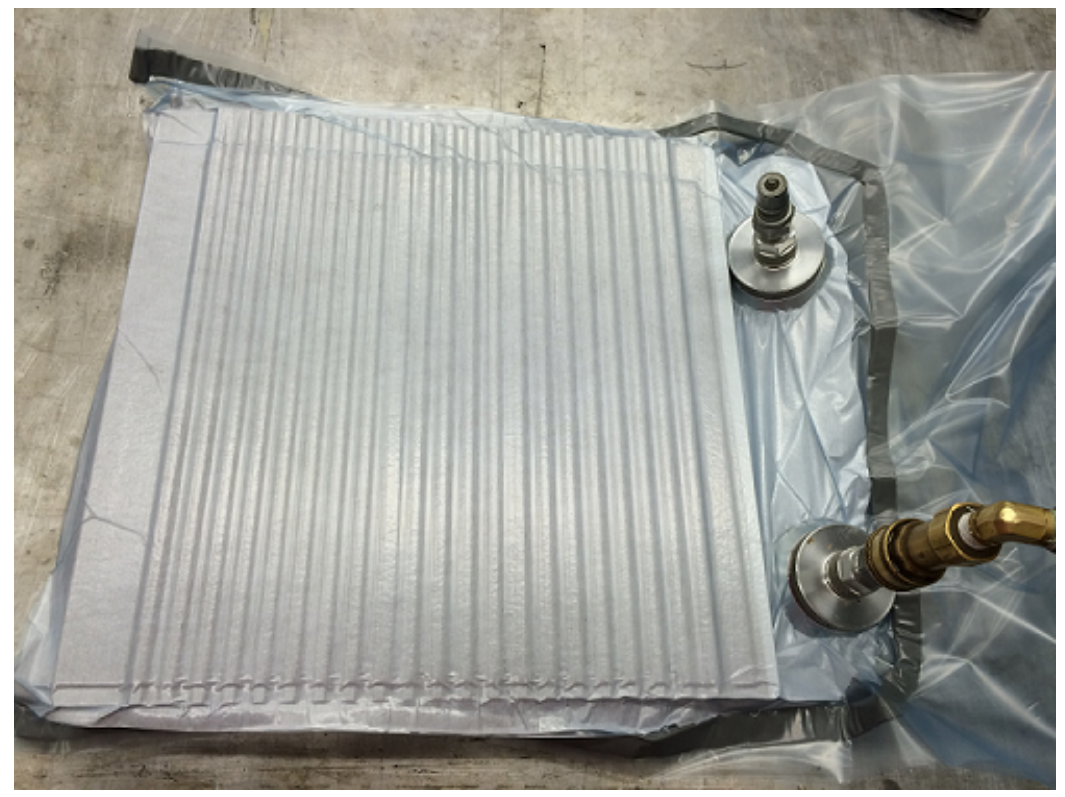

Figure 3.8: Sealed Vaccuum Bag with Corrugated Layup Under Pressure

For the carbon fiber coupons, five plies of the uni-directional pre-preg were laid together prior to application to the mold. From experimentation, I discovered that 
this material contoured and stuck to the mold better without any additional heat to the mold. On the contrary, heating the mold caused the material to relax out of the grooves. I experimented with 8 and 6-ply layups for this carbon fiber. Layup onto the mold was easier with less plies. To keep the coupon thickness close to that of the fiberglass coupons, I decided to reduce the number of plies to 5 . The final layup sequence for these coupons was $[0 / 90 / \overline{0}]_{s}$, where the reference axis was in the transverse direction. The layers within the vacuum bag were identical to the corrugated fiberglass vacuum bag. Layups without the porous material had an excess of epoxy in the corners as previously mentioned. The porous layer was able to absorb some of the epoxy and assist in obtaining a trapezoidal geometry. Initially, a layer of peel-ply was applied instead of the porous sheet, but the peel-ply failed to separate cleanly from the part.

After the layup cured, the corrugated panel was cut into the dimensions corresponding to the different experimental tests shown in Table 3.5. Tensile test coupons had a length of 10 inches and width of 1 inch. Bend test coupons had a length of 10 inches and width of 1 inch. Aluminum tabs were necessary for the tensile test coupons to prevent crushing and slippage in the Instron grips. The aluminum tabs were 2 in. by 1 in. and 0.065 inches thick. Magnolia structural adhesive was used to fill in the corrugations between the top and bottom tabs on each end. Samples of corrugated coupons with tabs are shown in Figure 3.9. The tabs were cured under 200 pounds of force and heated in the press for an hour. The coupons were then ready for testing.

Table 3.5: Dimensions of Corrugated Test Coupons

\begin{tabular}{|c|c|c|c|c|}
\hline Material & Direction & Length[in] & Width [in] & Thickness [in] \\
\hline FG & Longitudinal & 10.0 & 1.0 & 0.04 \\
FG & Transverse & 10.0 & 1.0 & 0.04 \\
CF & Longitudinal & 10.0 & 1.0 & 0.044 \\
CF & Transverse & 10.0 & 1.0 & 0.044 \\
\hline
\end{tabular}




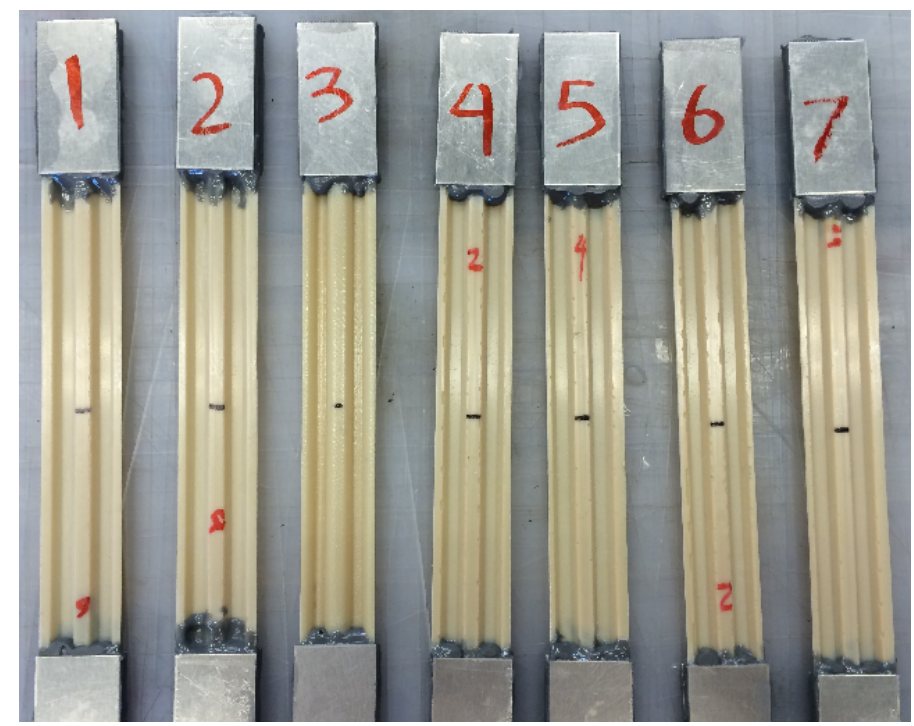

Figure 3.9: Corrugated Coupons with Aluminum Tabs

\subsection{Composite Material Cure Cycles}

The test coupons were manufactured from two different types of composites. Both materials were pre-impregnated with epoxy and had to be cured with heat. The Fiberglass (FG) was a weave while the Carbon fiber $(\mathrm{CF})$ was uni-directional tape. The materials available to the lab were all donations from companies so the material options are limited.

\subsubsection{Fiberglass Cure Cycle}

The fiberglass used in this research is Cytec MXB 7701/7781, which is a preimpregnated satin weave. This fiberglass was cured in the autoclave at a pressure of 45 psi. First step, the temperature was increased at $5^{\circ} \mathrm{F}$ per minute until the temperature reached $250^{\circ} \mathrm{F}$. Second step, the material cured at $250^{\circ} \mathrm{F}$ for 90 minutes. Lastly, the temperature was lowered at $5^{\circ} \mathrm{F}$ per minute until the temperature dropped to $140^{\circ} \mathrm{F}$. A visual representation of the cure cycle is shown in Figure 3.10 . 


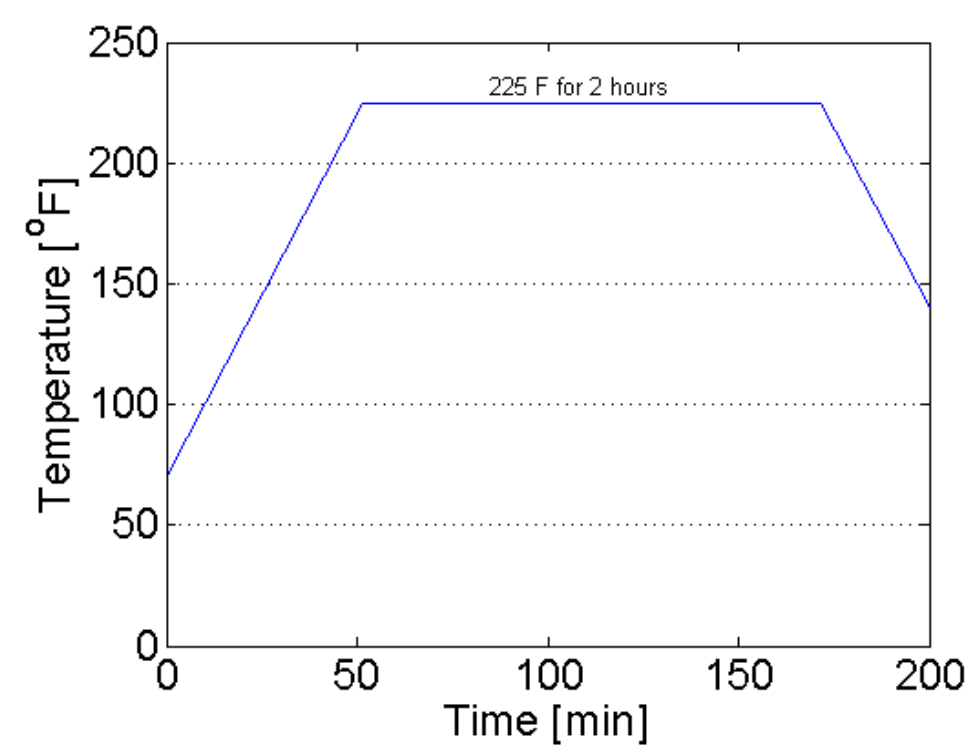

Figure 3.10: Cytec MXB 7701 Cure Cycle

\subsubsection{Carbon Fiber Cure Cycle}

Two carbon fiber uni-directional tapes were considered for use in making the coupons. The final carbon fiber material chosen for this research was M46J/TC250 unidirectional carbon fiber tape. The Newport 301 (NCT301) TR50S was initially considered because a full roll of the material was available. NCT301 had a flexible cure so the same cure cycle as the Cytec FG could be used even though it is not the recommended cure cycle. By using the same cure cycle, both materials could have been cured concurrently to save manufacturing time.

Flat coupons were manufactured with NCT301 and tested while I waited for the aluminum mold to be machined. However, it was not until the first layup on the corrugated mold that I realized the NCT301 tape lacked the tackiness to stay on the mold. Fortunately, a fellow graduate student finished manufacturing her parts and had leftover M46J/TC250 CF uni-directional tape. After testing a layup to make corrugated composites, I decided the M46J/TC250 had the appropriate tackiness to ease the layup process.

The Tencate TC250/M46J was used to manufacture the CF coupons. A visual 
representation of the cure cycle is shown in Figure 3.11. The layup was cured in the autoclave under a pressure of $40 \mathrm{psi}$. First, the temperature was increased at $3^{\circ} \mathrm{F}$ per minute until the temperature reaches $185^{\circ} \mathrm{F}$. At this stage, the material was cured at $185^{\circ} \mathrm{F}$ for 45 minutes. The second stage requires a ramp rate of $3^{\circ} \mathrm{F}$ per minute to a temperature of $265^{\circ} \mathrm{F}$. The material was cured at $265^{\circ} \mathrm{F}$ for 2 hours before ramping down at $5^{\circ} \mathrm{F}$ per minute.

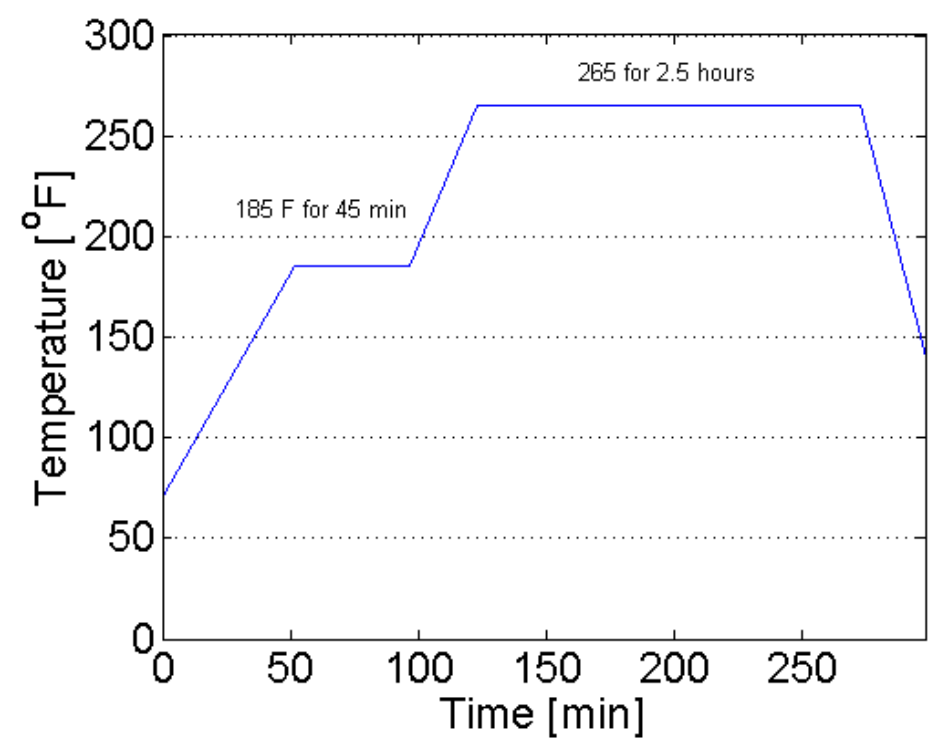

Figure 3.11: Tencate TC250 Cure Cycle 


\section{CHAPTER 4: ANALYTICAL MODEL}

This chapter explains the basic stiffness equations for composite laminates as well as the analytical methods used to calculate extensional and flexural stiffnesses of corrugated specimens. The methods include Castigliano's from Golzar[6] and equivalent models from Xia and Friswell[9]. Based on the geometry of one trapezoid unit, the stiffness properties may be calculated analytically. These results will be compared with experimental and FEA results in Chapter 7 .

\subsection{Mechanics of Composite Laminates}

Stiffness properties of composite laminates are calculated with basic mechanics of composite equations. To use the following equations from Reference [10], basic assumptions are made about the laminate:

- Laminate thickness is very small.

- Plies in the laminate are perfectly bonded.

- Lines perpendicular to the laminate surface is consistent with the surface after deformation.

- The laminate is linear elastic.

- Stresses and strains in the thickness is negligible.

Stress can be arranged as a function of strain such that

$$
\left[\begin{array}{c}
\sigma_{1} \\
\sigma_{2} \\
\tau_{12}
\end{array}\right]=\left[\begin{array}{lll}
Q_{11} & Q_{12} & Q_{16} \\
Q_{12} & Q_{22} & Q_{26} \\
Q_{16} & Q_{26} & Q_{66}
\end{array}\right]\left[\begin{array}{c}
\epsilon_{1} \\
\epsilon_{2} \\
\gamma_{12}
\end{array}\right]
$$


where $Q$ 's are reduced stiffnesses:

$$
\begin{aligned}
Q_{11} & =\frac{E_{1}}{1-\nu_{12} \nu_{21}}, \\
Q_{22} & =\frac{E_{2}}{1-\nu_{12} \nu_{21}}, \\
Q_{12} & =\frac{\nu_{12} E_{2}}{1-\nu_{12} \nu 21}, \\
Q_{66} & =G_{12},
\end{aligned}
$$

and for specially orthotropic laminates,

$$
Q_{16}=Q_{26}=0
$$

The reduced stiffnesses are calculated using ply properties $E$ and $G$. The lamina stiffness matrix is defined as

$$
[\bar{Q}]=[T]^{-1}[Q]\left[\begin{array}{lll}
1 & 0 & 0 \\
0 & 1 & 0 \\
0 & 0 & 2
\end{array}\right][T]
$$

where $T$ is the transformation matrix. The grand vector matrix for composite laminates,

$$
\left[\begin{array}{l}
N \\
M
\end{array}\right]=\left[\begin{array}{ll}
A & B \\
B & D
\end{array}\right]\left[\begin{array}{l}
\epsilon \\
K
\end{array}\right],
$$

consists of stiffness matrices, $A, B$, and $D . N$ and $M$ are stress and moment resultants, respectively, while $\epsilon$ is strain and $K$ is curvature. The extensional stiffness matrix, $A$, is defined as

$$
A_{i j}=\sum_{k=1}^{n}\left[\bar{Q}_{i j}\right]_{k}\left(h_{k}-h_{k-1}\right)
$$


where $i$ and $j$ represent the direction in $\mathrm{x}$ and $\mathrm{y}$ axes, $k$ is the ply number, and $h$ is the distance from the midplane of the laminate.

The coupling stiffness matrix is defined as:

$$
B_{i j}=\frac{1}{2} \sum_{k=1}^{n}\left[\bar{Q}_{i j}\right]_{k}\left(h_{k}{ }^{2}-h_{k-1}^{2}\right)
$$

The bending stiffness matrix is defined as:

$$
D_{i j}=\frac{1}{3} \sum_{k=1}^{n}\left[\bar{Q}_{i j}\right]_{k}\left(h_{k}{ }^{3}-h_{k-1}{ }^{3}\right)
$$

The stiffness in the x-direction, $E_{x}$, is calculated using elements in the extensional stiffness matrix and thickness of the laminate, $t$, as follows:

$$
E_{x}=\frac{A_{11}}{t}+\frac{A_{12}}{t}\left(\frac{A_{26} A_{16}-A_{12} A_{66}}{A_{22} A_{66}-A_{26}{ }^{2}}\right)+\frac{A_{16}}{t}\left(\frac{-A_{16}}{A_{66}}+\frac{A_{26} A_{12} A_{66}-A_{26}{ }^{2} A_{16}}{A_{22} A_{66}{ }^{2}-A_{26}{ }^{2} A_{66}}\right) .
$$

The stiffness in the y-direction, $E_{y}$, is similarly calculated using elements in the extensional stiffness matrix and thickness of the laminate as follows:

$$
E_{y}=\frac{A_{12}}{t}\left(\frac{A_{16} A_{26}-A_{12} A_{66}}{A_{11} A_{66}-A_{16}{ }^{2}}\right)+\frac{A_{22}}{t}+\frac{A_{26}}{h}\left(\frac{-A_{26}}{A_{66}}+\frac{A_{16} A_{12} A_{66}-A_{16}{ }^{2} A_{26}}{A_{11} A_{66}{ }^{2}-A_{16}{ }^{2} A_{66}}\right)
$$

The tensile stiffnesses in the transverse and longitudinal directions for the flat specimens are calculated using Eq. (4.8) and (4.9). The bending stiffness in the transverse direction and longitudinal directions are defined as

$$
\begin{aligned}
& D_{t}=D_{11} \text { and } \\
& D_{l}=D_{22} .
\end{aligned}
$$


Methods to predict the stiffness properties of corrugated composites will be discussed in the following sections.

\subsection{Bernoulli-Euler Beam Method}

One of the analytical methods used to predict the tensile and flexural properties for corrugated beams is using a Bernoulli-Euler beam based on Castigliano's theorem as used by Golzar[6].

The effective transverse (in the corrugation direction) tensile stiffness is

$$
E_{t}=\frac{P L}{W h \delta}
$$

where $P$ is the load, $L$ is the length of the corrugation (defined in the transverse direction), $W$ is the width of the corrugation (in the longitudinal direction), and $h$ is the thickness of the specimen. The deflection of the specimen, $\delta$, is defined as

$$
\delta=\frac{P\left(b_{1}+b_{3}\right)}{A_{11} w}+\frac{2 P b_{2} h^{2}}{3 D_{11} w}+\frac{P b_{1} h^{2}}{D_{11} w}+\frac{2 P(\sin \phi)^{2} b_{2}}{A_{11} w}
$$

where $b_{1}, b_{2}$, and $b_{3}$ are lengths of the trapezoid sides defined in Figure 4.1. $A_{11}$ and $D_{11}$ are elements in the extensional stiffness matrix and bending stiffness matrix, respectively, for the flat laminate calculated in Section 4.1.

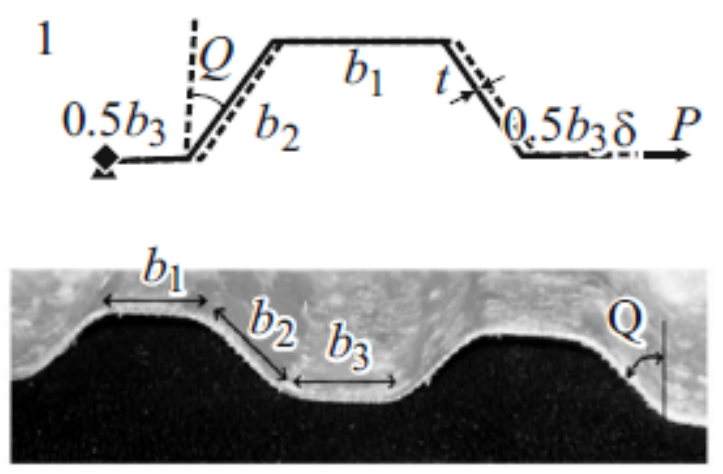

Figure 4.1: Trapezoidal Unit Geometry for Golzar Method[6] 
The moment of inertia for a trapezoid unit shown in Figure 4.1 is defined as

$$
\begin{aligned}
I= & \frac{b_{1} t^{3}}{12}+b_{1} t\left[\frac{h\left(b_{2}+b_{3}\right)}{b_{1}+2 b_{2}+b_{3}}\right]^{2}+\frac{b_{3} t^{3}}{12}+b_{3} t\left[\frac{h\left(b_{1}+b_{2}\right)}{b_{1}+2 b_{2}+b_{3}}\right]^{2}+ \\
& \frac{b_{2}(\sin \phi)^{2} t^{3}}{6}+\frac{b_{2}^{3}(\cos \phi)^{2} t}{6}
\end{aligned}
$$

where $\phi$ is the angle from the vertical and $t$ is the thickness of the composite layup.

$$
E_{l}=\frac{\left(b_{1}+2 b_{2}+b_{3}\right) A_{22}}{\left(b_{2} \cos \phi+t\right)\left(b_{1}+2 b_{2} \sin \phi+b_{3}\right)}
$$

where $A_{22}$ an element in the tensile stiffness matrix calculated in Section 4.1.

The effective flexural stiffness in the longitudinal direction is defined as

$$
D_{l}=\frac{I A_{22}}{t\left(b_{1}+2 b_{2} \sin \phi+b_{3}\right)}
$$

The effective flexural stiffness in the transverse direction is defined as

$$
D_{t}=\frac{\left(b_{1}+2 b_{2} \sin \phi+b_{3}\right) D_{11}}{b_{1}+2 b_{2}+b_{3}} .
$$

The bending and flexural stiffnesses calculated using this method are shown in Table 4.1.

Table 4.1: Stiffness Results from Bernoulli-Euler Beam Method

\begin{tabular}{|c|c|c|c|}
\hline Material & Direction & $\mathrm{E}[\mathrm{psi}]$ & $\mathrm{D}\left[\mathrm{in} \cdot \mathrm{lb} \mathrm{b}_{f}\right]$ \\
\hline FG & Longitudinal & $8.83 \mathrm{E} 5$ & 1027 \\
FG & Transverse & $6.09 \mathrm{E} 4$ & 13.9 \\
$\mathrm{CF}$ & Longitudinal & $3.75 \mathrm{E} 6$ & 4710 \\
$\mathrm{CF}$ & Transverse & $1.65 \mathrm{E} 5$ & 40.4 \\
\hline
\end{tabular}




\subsection{Xia and Friswell's Equivalent Model}

Another analytical method to predict the stiffness properties of corrugated composites is an equivalent model developed by Xia and Friswell [9]. This method was developed for two geometries including circular and trapezoidal corrugations. The model for trapezoidal corrugations is used for this research. The stiffness properties for a panel is determined from the basic unit cell of the corrugations. The trapezoidal unit cell geometry is shown in Figure 4.2. The proposed method redefines stiffness elements in the grand stiffness matrix of the composite laminate to create an equivalent plate model.

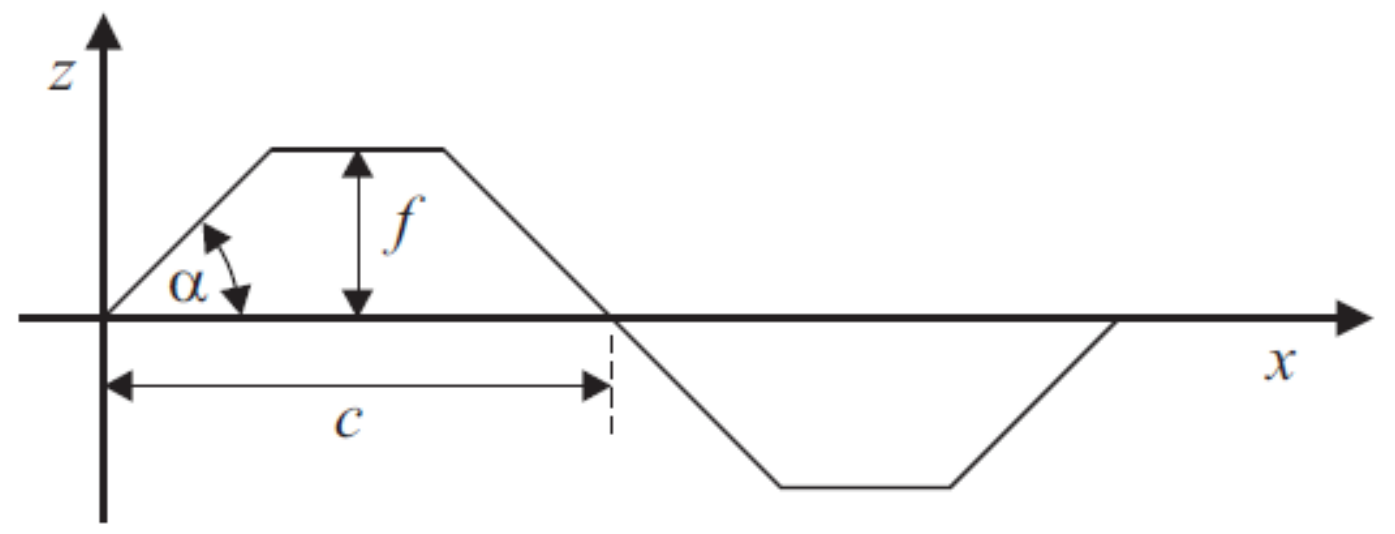

Figure 4.2: Trapezoidal Unit Geometry for Equivalent Models [9]

Extensional stiffness matrix term , $\bar{A}_{11}$, of the equivalent plate model is defined as

$$
\bar{A}_{11}=\frac{c}{\left(\frac{f \cos ^{2} \alpha}{\sin \alpha A_{11}}+\frac{f^{3}}{3 \sin \alpha D_{11}}\right)+\left(c-\frac{2 f}{\tan \alpha}\right)\left(\frac{1}{A_{11}}+\frac{f^{2}}{\left.D_{11}\right)}\right.}
$$

where $c$ is half the width of one trapezoid unit, $f$ is half the height of one trapezoid unit, $\alpha$ is the angle from the horizontal, $A_{11}$ and $D_{11}$ are the extensional and bending stiffness elements of the flat composite laminate, respectively. See Section 4.1 for details on the stiffness matrices. 


$$
\begin{aligned}
& \bar{A}_{22}=\frac{\frac{2 f}{\sin \alpha}+c-\frac{2 f}{\tan \alpha}}{c} A_{22} \\
& \bar{A}_{66}=\frac{c}{\frac{2 f}{\sin \alpha}+c-\frac{2 f}{\tan \alpha}} A_{66}
\end{aligned}
$$

The new bending stiffness matrix elements are calculated as follows:

$$
\begin{gathered}
\bar{D}_{11}=\frac{c}{\frac{2 f}{\sin \alpha}+c-\frac{2 f}{\tan \alpha}} D_{11}, \\
\bar{D}_{22}=\frac{\frac{4 f^{3}}{3 \sin \alpha}+2 f^{2}\left(c-\frac{2 f}{\tan \alpha}\right)}{c} A_{22},
\end{gathered}
$$

and

$$
\bar{D}_{66}=\frac{\frac{2 f}{\sin \alpha}+c-\frac{2 f}{\tan \alpha}}{c} D_{66} .
$$

To compare the analytical results with the experimental, the Xia and Friswell extensional stiffness terms above were converted to pounds-force per square inch. The tensile stiffness per length is calculated using Eq. (4.8) and (4.9). The units for the tensile stiffness will be pounds-force per square inch while the units for bend stiffness will be pounds-force inch. The calculated stiffnesses using this method are shown in Table 4.2.

Table 4.2: Stiffness Results from Friswell Method

\begin{tabular}{|c|c|c|c|}
\hline Material & Direction & $\mathrm{E}[\mathrm{psi}]$ & $\mathrm{D}\left[\right.$ in $\left.\cdot l b_{f}\right]$ \\
\hline FG & Longitudinal & $7.18 \mathrm{E} 5$ & 1969 \\
FG & Transverse & $1.14 \mathrm{E} 4$ & 13.2 \\
$\mathrm{CF}$ & Longitudinal & $4.88 \mathrm{E} 6$ & 8990 \\
$\mathrm{CF}$ & Transverse & $4.96 \mathrm{E} 4$ & 38.3 \\
\hline
\end{tabular}




\section{CHAPTER 5: FINITE ELEMENT ANALYSIS}

This chapter covers the process of building finite element models. A model was built for each type of specimen with boundary conditions corresponding to the test setups. The models were created and analyzed in ABAQUS CAE 6.14. Results from finite element analysis will be compared with experimental results in later chapters.

\subsection{FEA Tensile Test Model}

Model development and analysis for tensile specimens are discussed in this section. Tensile specimens were modeled without external fixtures because simple boundary conditions were sufficient. The following subsections describe the steps to build the model in ABAQUS from creating the geometry to applying boundary conditions.

\subsubsection{Tensile Specimen: Geometry}

The models were created as 3D, deformable, solid elements. Only the gage section of the test specimens will be modeled. The clamped areas of the specimen will be simulated by the boundary conditions. Thus, the geometry and dimensions will differ from the test specimens. The dimensions of the flat specimens are outlined in Table 5.1 and the dimensions of the corrugated specimens are shown in Table 5.2. The corrugated longitudinal specimens contain 13 trapezoid units while the transverse specimens contain 2 units. Refer to Section 2.3 for the geometry of the trapezoid unit.

\subsubsection{Tensile Specimen: Material and Section Assignment}

Despite having different geometries for flat and corrugated models, the process of assigning a material and section to the part was identical. Each part was defined 


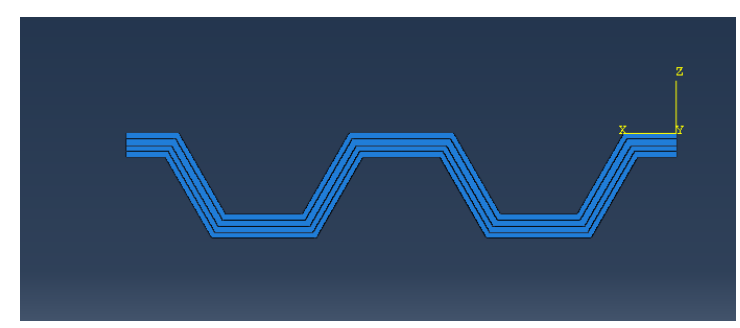

Figure 5.1: Cross Section of Tensile Corrugated FG FEA Model

Table 5.1: Dimensions of Flat FEA Models

\begin{tabular}{|c|c|c|c|c|}
\hline Specimen Type & Direction & Length [in] & Width [in] & Thickness \\
\hline FG & Longitudinal & 6.0 & 1.27 & 0.04 \\
FG & Transverse & 8.28 & 1.0 & 0.04 \\
CF & Longitudinal & 6.0 & 1.27 & 0.044 \\
CF & Transverse & 8.28 & 1.0 & 0.044 \\
\hline
\end{tabular}

as a solid composite part with the composite layup tool. The composite layup tool in ABAQUS was used to specify the material and orientation of each ply in the layup. The section was assigned by selecting the entire part as the region. In ABAQUS, the dimensions are unitless so it is important to keep units consistent. The geometry and material properties are all in English units. Each material was defined as elastic engineering constants with properties specified in Appendix A. The material orientation must be specified for each section. An example is shown in Figure 5.2 below.

\subsubsection{Tensile Specimen: Assembly and Step}

The assembly was created by importing an instance of the part and orienting it in the xy-plane. For tensile specimens, the part was the only instance necessary in the assembly. Fixtures were unnecessary because of the boundary conditions defined in the next section. A static step was created because the specimens were only tested under static loads. 
Table 5.2: Dimensions of Corrugated FEA Models

\begin{tabular}{|c|c|c|c|c|}
\hline Specimen Type & Direction & Length [in] & Width [in] & Thickness \\
\hline FG & Longitudinal & 6.0 & 0.951 & 0.18 \\
FG & Transverse & 6.18 & 1.0 & 0.18 \\
CF & Longitudinal & 6.0 & 0.951 & 0.184 \\
CF & Transverse & 6.18 & 1.0 & 0.184 \\
\hline
\end{tabular}

\subsubsection{Tensile Specimen: Boundary Conditions and Loads}

The accuracy in which the numerical results match with the experimental results may be affected by the boundary conditions applied on the model. This section specifies the boundary conditions for the tensile FEA models. The top of the specimen was fixed with an encastre boundary condition. The bottom of the specimen was fixed in the $\mathrm{x}$ - and $\mathrm{z}$-directions with a degree of freedom in the y-direction (along length of specimen). Refer to Figure 5.3 to see the boundary conditions. The load was applied at the bottom of the specimen as a pressure force with the magnitude specified as a total force in the y-direction. ABAQUS automatically distributes the force on the area such that the total force on the specimen equates the desired force.

\subsubsection{Tensile Specimen: Mesh}

The initial mesh was very rough to ensure that the analysis generated a solution, which was on the same order as what was expected. The first step was to specify the maximum element size for setting up global seeds. For corrugated specimens, part seeds must be specified in the corrugation direction. There was more variability in this direction so a higher density of elements was necessary for a converged solution. After the seeds were set, the parts can be meshed automatically using the default element type. The default mesh element for solids is type C3D8. Examples of rough and fine meshes are shown in Figure 5.4.

The mesh was refined by increasing the number elements and this generates 


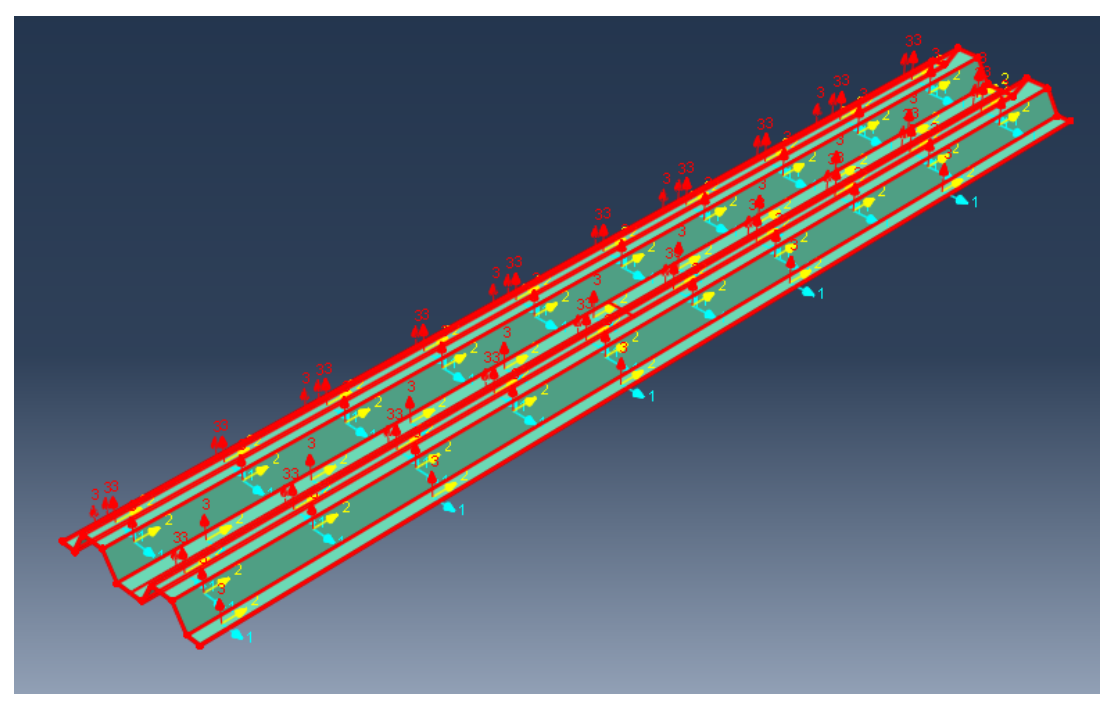

(a) Isometric View

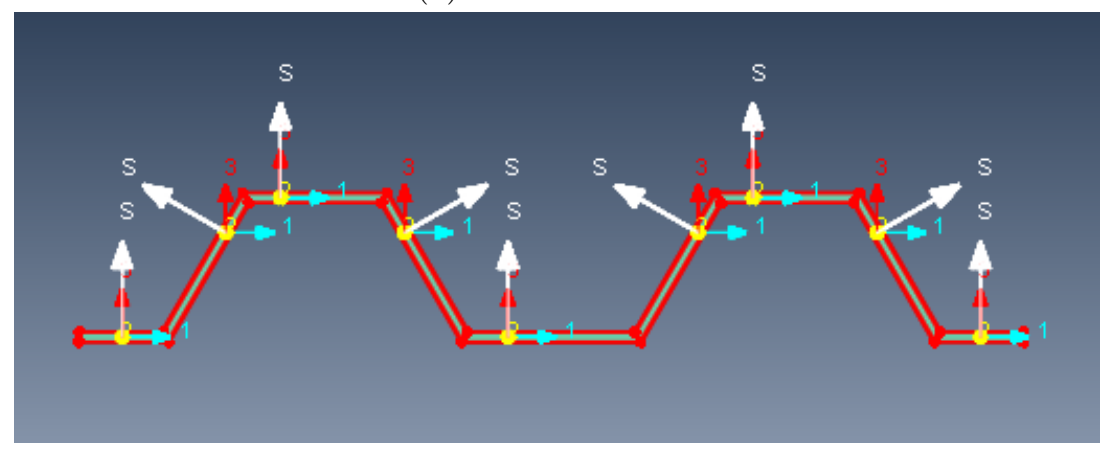

(b) Ply Stacking Direction

Figure 5.2: Material Orientation of Corrugated FEA Model

more accurate results. Mesh convergence studies were done to check solutions for convergence. The deflection of the models was the solution that defined convergence. Refer to Appendix C for plots of mesh convergence studies for tensile specimens. As expected, the mesh for corrugated transverse models require a greater refinement. As the mesh density increases, the solution converges. More elements mean more computation time. Therefore, mesh refinement was no longer necessary when the solution was converged. 


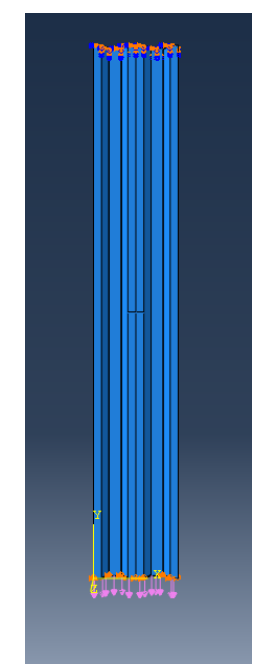

Figure 5.3: Boundary Conditions on Corrugated Tensile FEA Model

\subsubsection{Tensile Specimen: Data Analysis}

Post-processing was completed by ABAQUS to generate stress and strain data. However, the stress on corrugated specimens should be calculated based on the effective area similar to the experimental results. Stress was calculated using Eq. 5.1.

$$
\sigma=\frac{P}{A_{e f f}},
$$

where $P$ is the tensile load and $A_{e f f}$ is the effective Area. The effective area is calculated as

$$
A_{e f f}=w h
$$

where $w$ is the width of the specimen and $h$ is the height of the specimen.

Extensional stiffness is calculated as

$$
E=\frac{\sigma}{\epsilon}
$$

where $\sigma$ is stress and $\epsilon$ is the strain. 


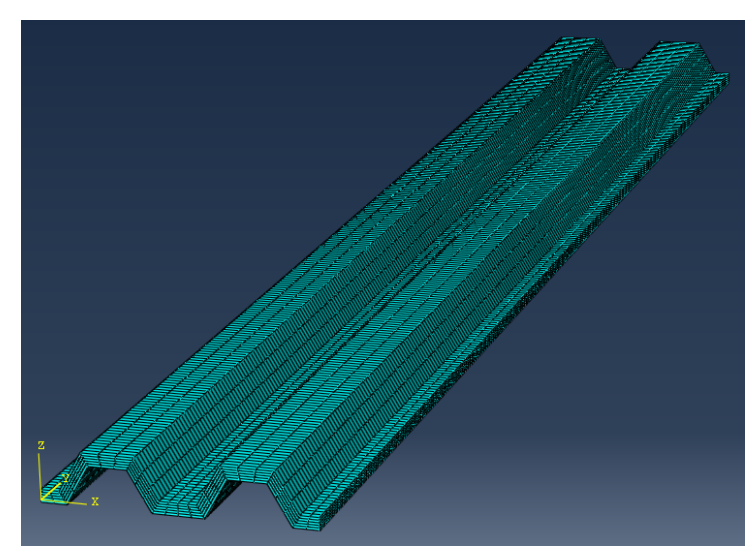

(a) Rough

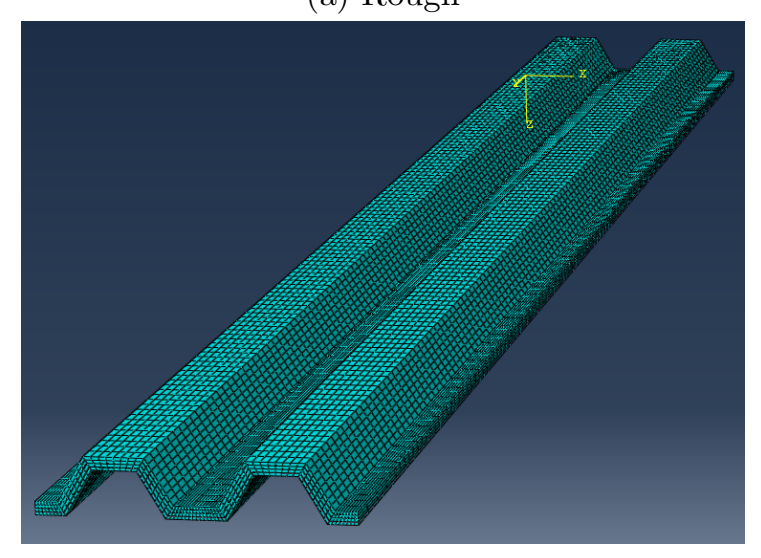

(b) Fine

Figure 5.4: Mesh Density of Corrugated Tensile FEA Model

The stress and strain data in direction 2 for a central element on the specimen was selected as shown in Figure 5.5. A central element was selected because that was the location where the extensometer measured strain data. Strain data was selected for a bottom element for all corrugated transverse models because the experimental data for this specimen type used the crosshead displacement.

Deformation in the load direction is shown for flat and corrugated CF specimens under tensile load in Figures 5.6 and 5.7, respectively. 

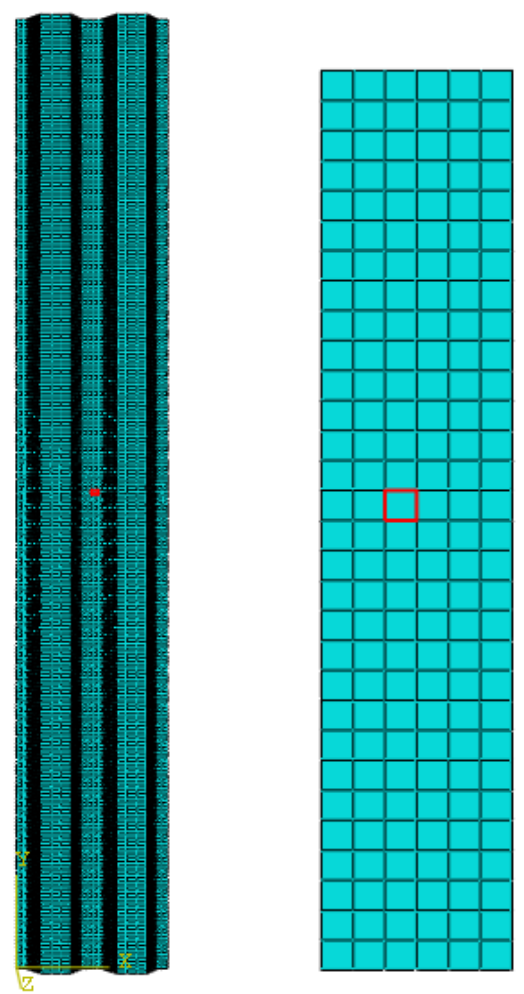

$\begin{array}{ll}\text { (a) Corrugated } & \text { (b) Flat Longitudi- } \\ \text { Longitudinal } & \text { nal }\end{array}$

Figure 5.5: Central Element Selection for Data Output
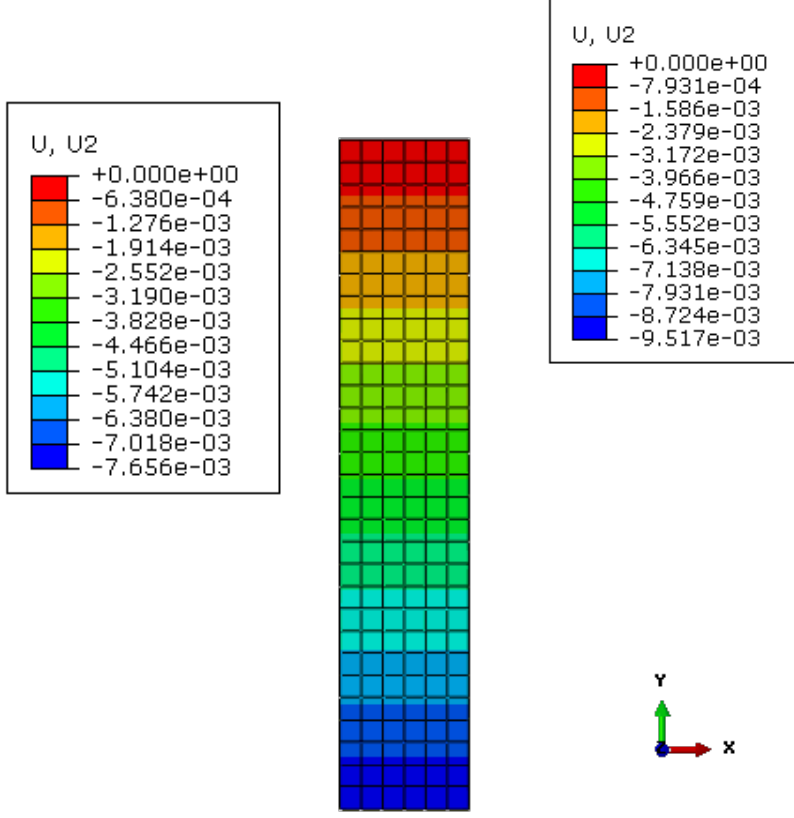

(a) Flat Longitudinal

(b) Flat Transverse

Figure 5.6: FEA Deformation of Flat Tensile CF Specimens 

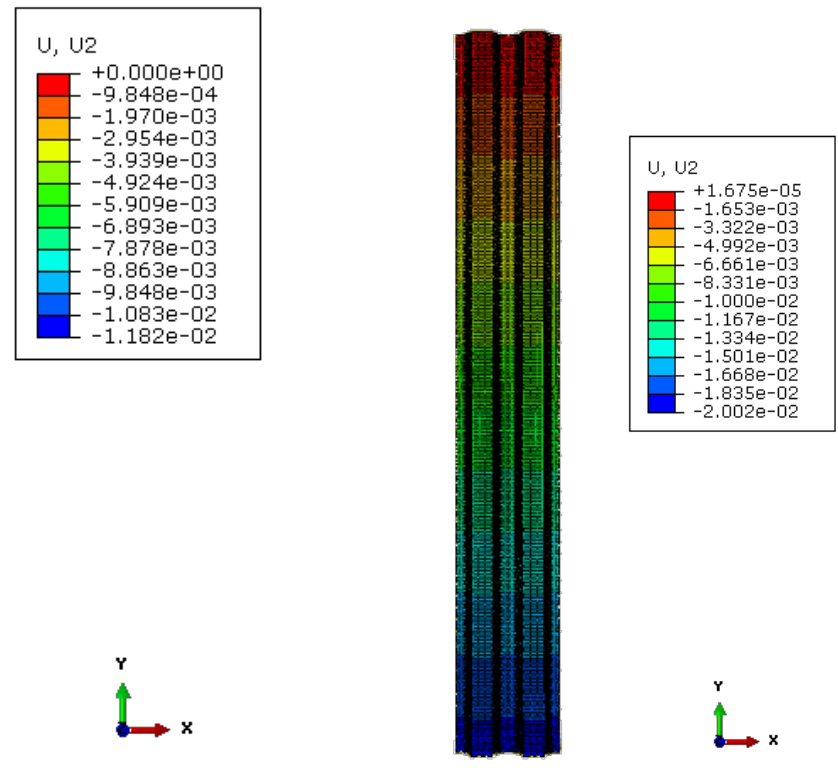

(a) Corrugated Longitudinal

(b) Corrugated Transverse

Figure 5.7: FEA Deformation of Corrugated Tensile CF Specimens 


\subsection{FEA Flexure Test Model}

Models of the bend test were more complicated than those of the tensile tests. The loading nose and support pins of the fixture were modeled as well. Interactions between the fixture and specimen had to be applied to observe the effects of the pins on the part. The part geometry and part mesh for flexure specimen models were similar to those of the tensile models. The details of the models are described in the following sections.

\subsubsection{Flexure Specimen: Geometry}

The entire composite test specimen was modeled for each specimen type and material. The dimensions of the models were the same as those in Tables 3.4 and 3.5. The longitudinal corrugated specimens had 2 trapezoid units while the transverse corrugated specimen had 21 trapezoid units. The composite parts were partitioned at the locations where there was contact with the loading and support pins.

The loading nose and support pins were identical so only one pin was modeled. The actual pins were cylindrical and had a 1-inch diameter and 2-inch length. Only half of the pin would make contact with the composite part, so half the pin was modeled. By modeling half of the pin, the total computation time was reduced. The parts were created with a sketch of the cross-section geometry and extrusion.

\subsubsection{Flexure Specimen: Material and Section Assignment}

The material and section assignments for the composite parts were exactly the same as the tensile models. Refer to Section 5.1.2 for details. The pins were steel so it was assigned a solid homogeneous section with isotropic properties defined in Appendix A. The steel pins were much stiffer in comparison to the composite specimens. The material properties of the pins were negligible because the pins will be defined as rigid bodies in the Interactions section. 


\subsubsection{Flexure Specimen: Assembly and Step}

A static step was created for the assembly because only a static load was applied to the model. The assembly was composed of one instance of each ply and three instances of the pin. First, the composite part was imported followed by the pins. The composite part was imported first because the pins were to be aligned to it. Position constraints including the edge-to-edge and parallel face constraints were applied to perfectly align the pins. The assembly is shown in Figure 5.8. Each ply part was partitioned at locations where there was contact between the composite and the pins. The parts were also partitioned 1 inch past the two support pins. Partitioning of the parts allow for a higher density of elements in the gage section where the most deformation occurs.

\subsubsection{Flexure Specimen: Boundary Conditions, Loads, and Interactions}

Boundary conditions applied to the model must represent the experimental test set-up. Wrong boundary conditions and loads affect the FEA results in comparison to experimental results. The top pin was fixed with an encastre boundary condition. The two support pins were constrained in the $\mathrm{x}$ and $\mathrm{y}$ directions but free in the $\mathrm{z}$ direction. The load was applied in the positive $\mathrm{z}$ direction to the support pins in the same manner as the test set-up. The boundary conditions are shown in Figure 5.9. In addition to boundary conditions and loads, interactions were defined for the flexure models between the pins and composite parts. The pins were defined as rigid bodies because it was infinitely rigid compared to the composite part.

The interaction was set up as frictionless and hard contact, which allowed for separation post-contact. The interaction properties for the transverse corrugated CF specimens were different due to slipping that occurred between the pins and the composite. The interaction was set as rough and hard contact. The rough setting 


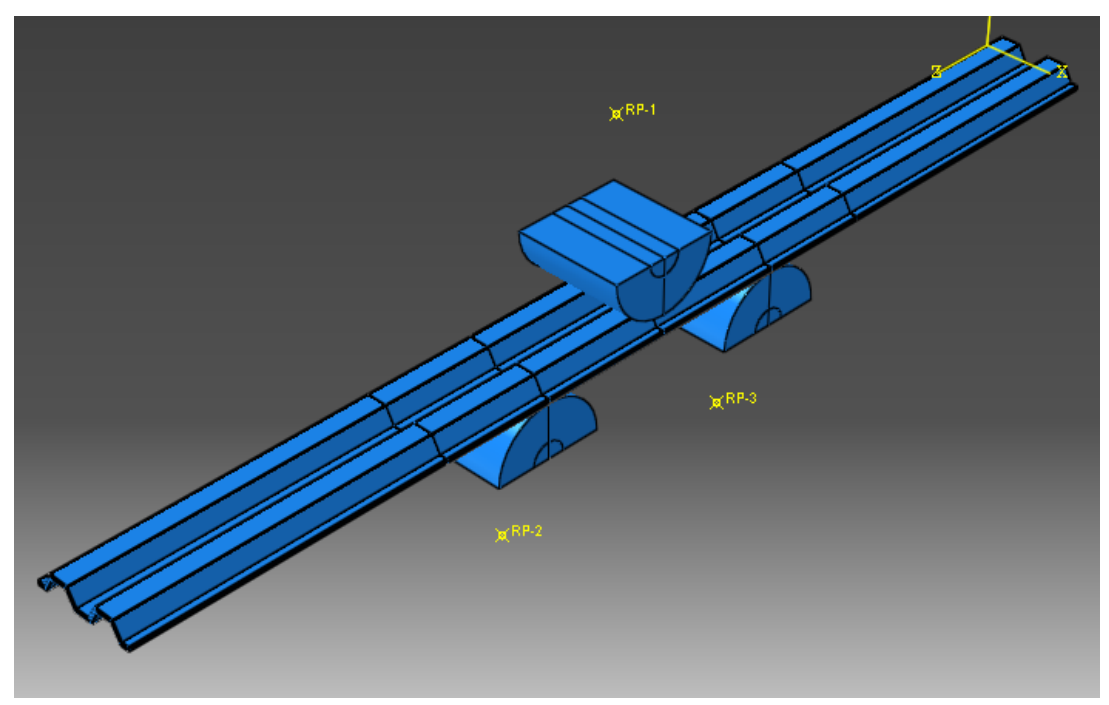

(a) Corrugated Longitudinal

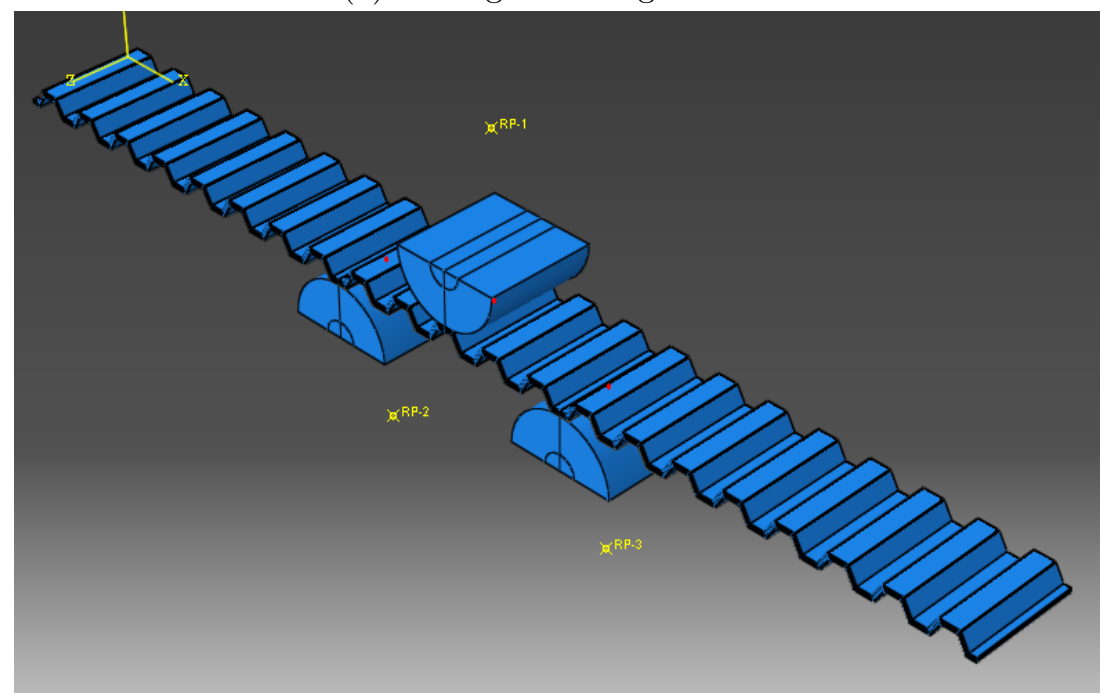

(b) Corrugated Transverse

Figure 5.8: Finite Element Flexure Model Assembly

maintains the point of contact between the pins and the composites layup. Besides the interaction properties, the interaction definitions were the same between all flexure models. The pins were selected as the master surface while the surfaces on the composite part were defined as the slave surface. Plies of the composite layup were constrained as perfect bonds so delamination will not occur. This condition was true for the linear region, which is sufficient for the scope of this research. 


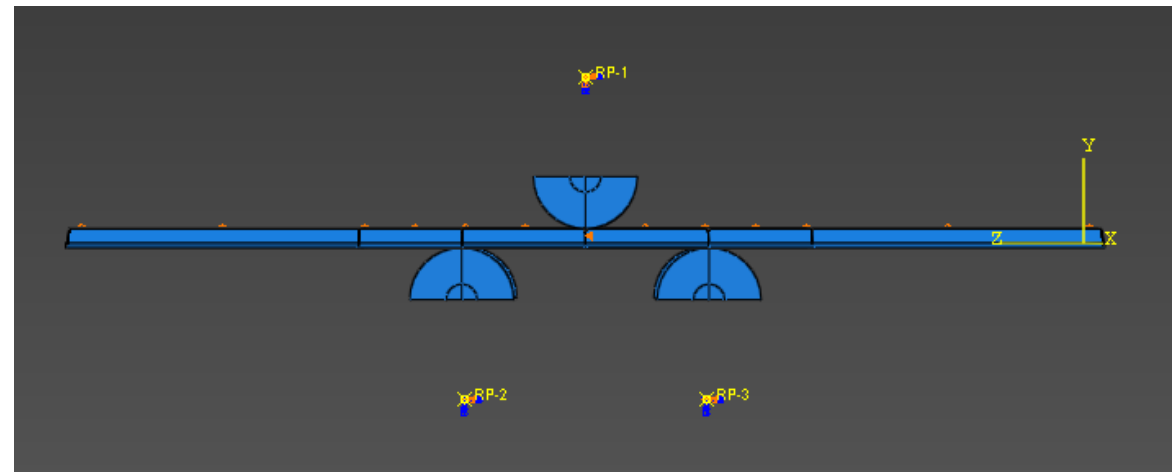

(a) Corrugated Longitudinal

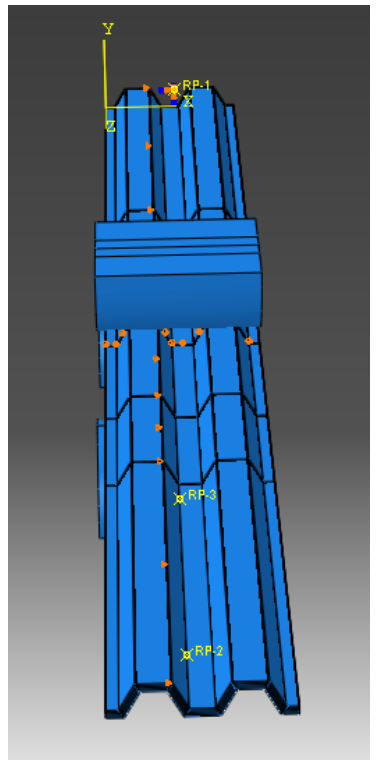

(b) Corrugated Transverse

Figure 5.9: Boundary Conditions on Corrugated Flexure FEA Model

\subsubsection{Flexure Specimen: Mesh}

Each instance in the assembly was meshed separately and the mesh was dependent on the parts. For composite parts, the mesh was composed of solid C3H8 elements. A higher density of elements was put in the gage section between the two lower pins. The gage section is the region where significant changes in stress and deflection occur. Thus, the analysis will converge faster with the same number of elements. Adding elements outside of the gage section does not greatly affect the solution. Elements were added in the gage section during the mesh convergence studies. 
See Appendix C for plots of mesh convergence studies. Elements were added by applying global seeds to the entire part and edge seeds on select edges in the gage section. The number of seeds were specified either by the desired minimum element size or by the number of seeds along the edge. Figure 5.10 shows the increased density of elements in the gage section.

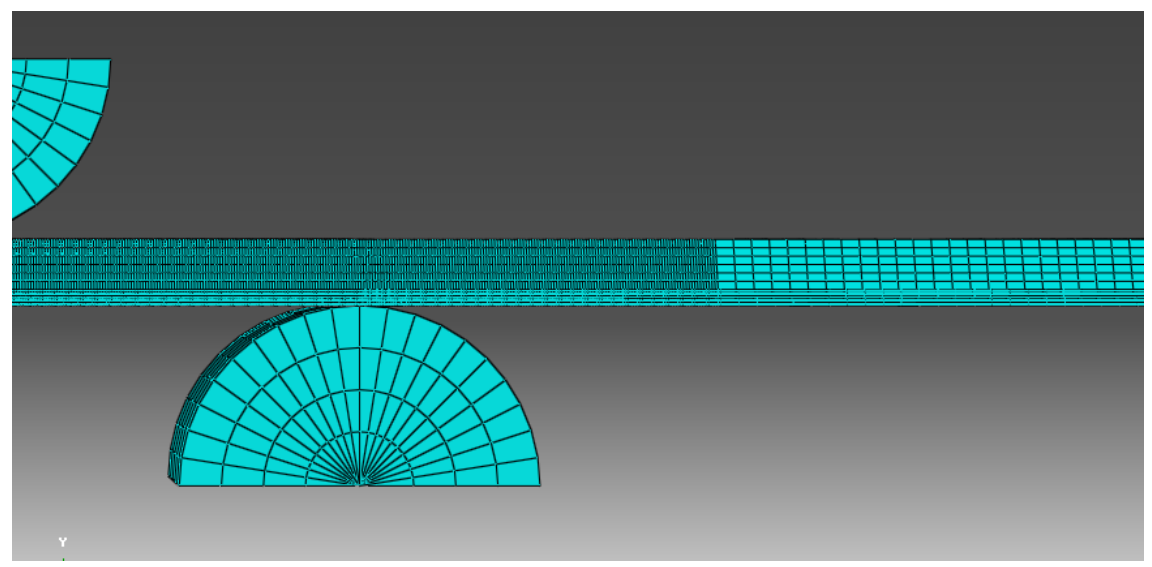

Figure 5.10: Mesh Density in Different Sections of Flexure FEA Model

The pin was meshed as a dependent part using wedge and hexagonal elements. The elements close to its center were defined as wedge elements in Mesh Controls. The remaining elements were defined as hex elements. Edge seeds were specified to create the mesh shown in Figure 5.11.

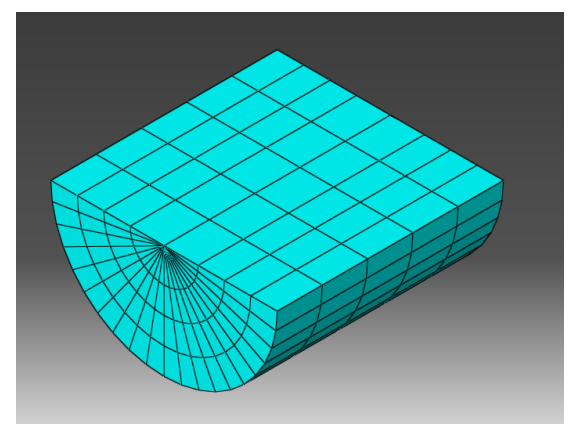

Figure 5.11: Pin Meshed with Wedge and Hexagonal Elements 


\subsubsection{Flexure Specimen: Data Analysis}

After running the analysis, the reaction load at the top pin and the deflection were collected for each case. The reaction load on the top pin in direction 2 was used to calculate flexural stiffness. The deflection on the support pins was also used. Flexural stiffness was calculated with the change in load per deflection. See Equation 5.4 for the calculation.

$$
E=\frac{\sigma}{\epsilon}
$$

where $m$ is the load per deflection in the linear region, $b$ is the width, $h$ is the height of the specimen.

The deformations of corrugated CF longitudinal and transverse specimens are shown in Figures 5.12. 

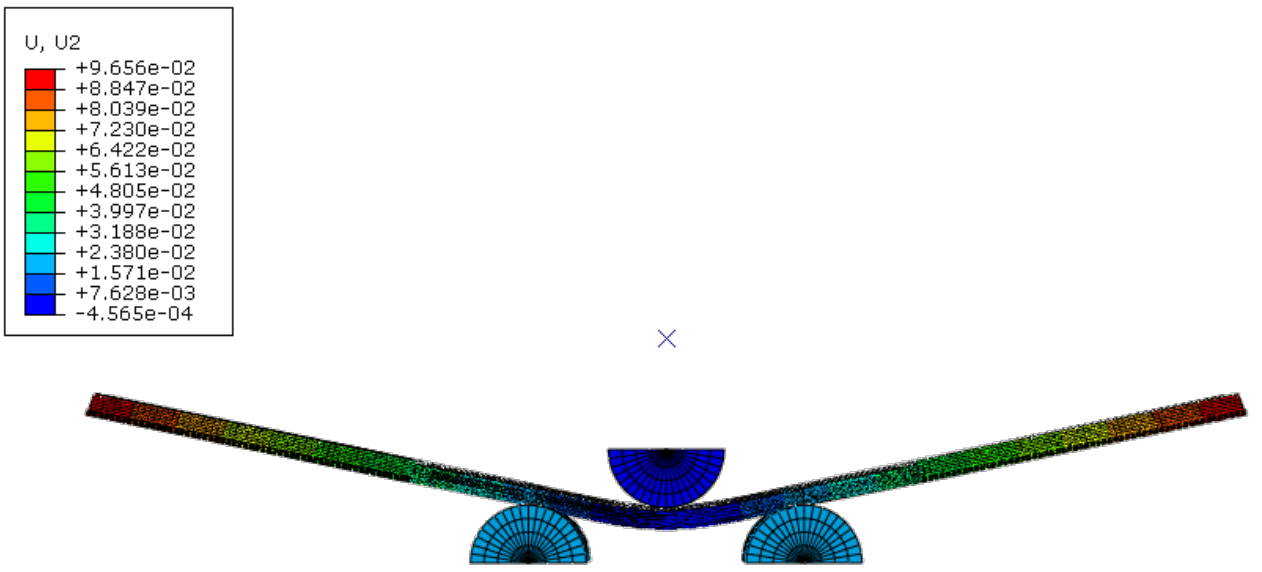

(a) Corrugated Longitudinal CF

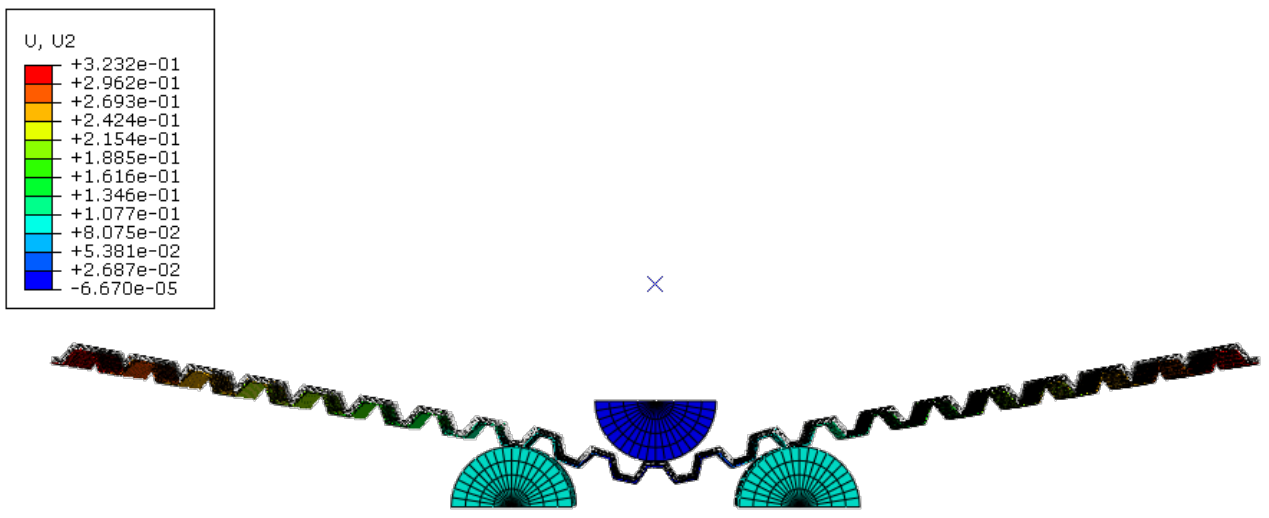

(b) Corrugated Transverse CF

Figure 5.12: FEA Deformation of Corrugated CF Specimens Under Flexural Load 


\section{CHAPTER 6: EXPERIMENTAL SET-UP AND TESTING}

In this chapter, the test procedures applied in this research will be discussed. There were different test procedures for each material property test. Those procedures will be described separately from the experimental test procedures. The tests and referenced ASTM standards are summarized in Table 6.1. The material test procedures are found in Section 6.1. Flat and corrugated specimens were tested using the same test procedures for the uniaxial tension test and 3-point bend test. The experimental test procedures will be described in Section 6.2.

Table 6.1: Types of Experimental Tests

\begin{tabular}{|c|c|c|}
\hline Test & ASTM Referenced & Properties Determined \\
\hline Material Tension & D3039 & Tensile Modulus, Poisson's Ratio \\
Material Compression & D3410 & Compressive Modulus \\
Volume Fraction & D2584 & Fiber Volume Fraction \\
Uniaxial Tension & D3039 & Extensional Stiffness \\
3-point Bend & D7264 & Flexural Stiffness \\
\hline
\end{tabular}

\subsection{Material Properties Test Procedures}

This section describes the tests conducted to determine material properties of the composite. Each test requires specimens with different dimensions and preparation. The Instron was used in all the tests except for the volume fraction procedure. The Young's Modulus was determined under tensile and compressive load from tension and compression tests. The Poisson's ratio of the material was determined using strain gage data during tension tests. Details of the test procedures will be elaborated upon in the following subsections. 


\subsubsection{Tensile Test}

For uniaxial tensile tests, specimens were clamped in the grips of the Instron heads with a gage section of 6 inches per ASTM D3039. The heads must be aligned to properly apply load in the axial direction; otherwise, the specimen might twist. The Instron machine applied a tensile force on the specimens at a strain rate of 0.05 inches per minute. The load cell measured the force while the displacement came from the Instron heads. Young's Modulus was determined by the slope of the linear region of the stress-strain curve. There was a concern with the accuracy of the cross-head displacement of the Instron. For a more accurate method of measuring the strain of the specimens, either a strain gage or an extensometer may be used. The ultimate strength and elastic modulus for the tension tests were compared to the properties in the datasheet.

According to ASTM D3039, at least five specimens are required per test condition in order to attain consistent results in an experimental test. A minimum of six specimens were required per test involving an extensometer because the first specimen was tested without the extensometer. The first specimen was tested until ultimate failure to determine the ultimate load, which will be used to determine the load applied to the remaining specimens without damaging the extensometer. The maximum displacement that can be measured by the extensometer was 0.1 inches. The extensometer is a sensitive piece of equipment that could be easily damaged. Thus, the specimens were loaded up to $30 \%$ of the ultimate load and the test was stopped before reaching the maximum displacement of the extensometer as a safety measure. After the extensometer was removed, the test continued until ultimate failure. 


\subsubsection{Compression Test}

The compression test was very similar to the tension test where the only variation was the direction of the force applied. The compression test was based on ASTM D3410. Specimens were clamped in the grips of the Instron heads leaving a gage section of 0.5 inches. Again, the load was applied at a strain rate of 0.05 inches per minute. An example of an FG specimen in a compression test is shown in Figure 6.1. Both tension and compression tests were necessary because the material properties such as the Young's Modulus and ultimate strength vary slightly depending on the direction of the applied load. See Section 7.1 for a comparison of measured material properties and datasheet properties.

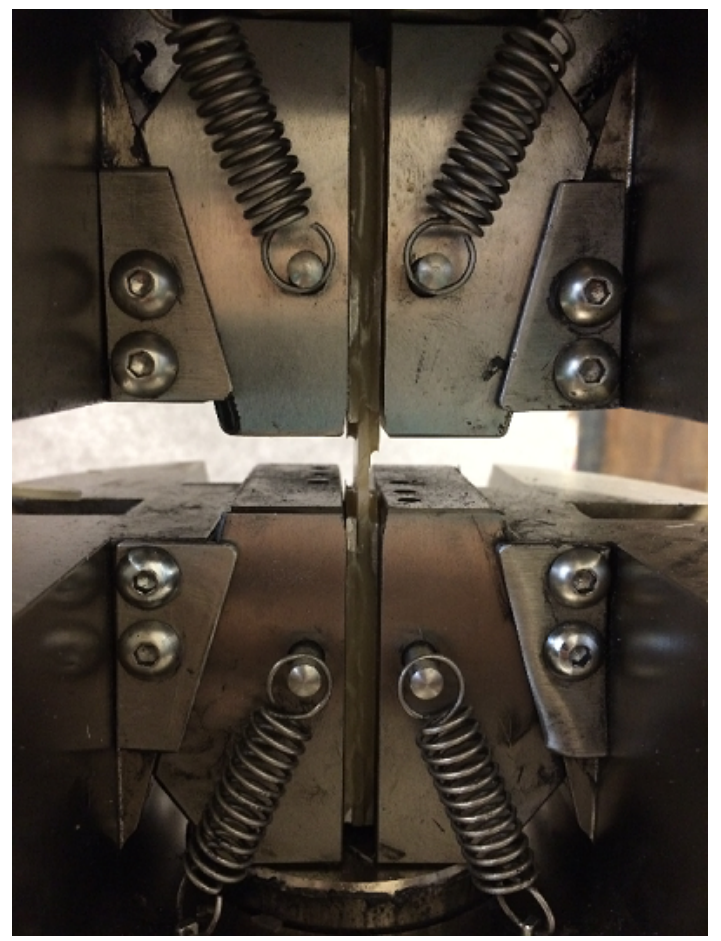

Figure 6.1: FG Specimen in Compression Test

\subsubsection{Poisson's Ratio}

This is the ratio that describes the interaction between the change in direction 1 due to a change in direction 2 of the material. Directions 1 and 2 are perpendicular 
to one another as shown in Figure 6.2. When a tensile specimen is loaded, there is tensile strain in direction 2 while there is compressive strain in direction 1 . The Poisson's ratio, $\nu$, is determined for each material with the following equation:

$$
\nu_{12}=-\frac{d \epsilon_{2}}{d \epsilon_{1}}
$$

Where $d \epsilon_{1}$ is the change in strain measured in direction 1 and $d \epsilon_{2}$ is the change in strain measured in direction 2 at the same time.

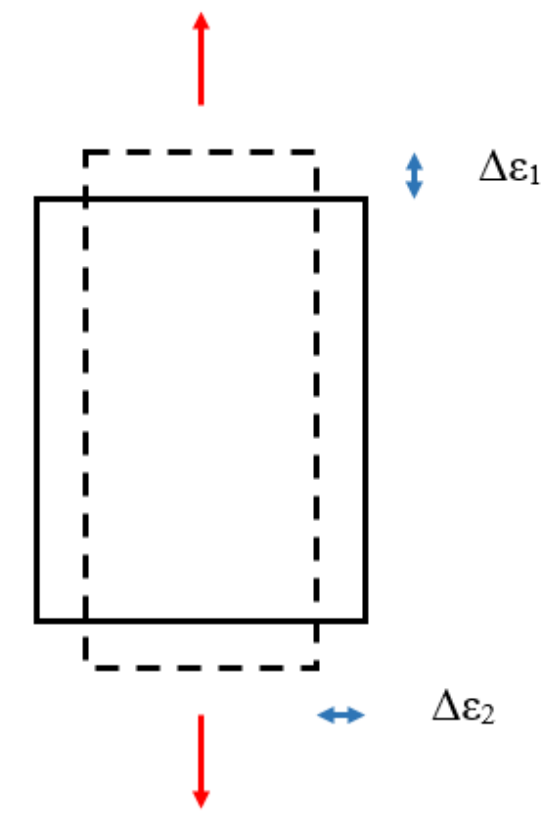

Figure 6.2: Poisson's Ratio for a Rectangular Section

To measure strain in both directions concurrently, strain gages were used for higher accuracy in the material tension test. To output data from the gages, the gages were connected to the Data Acquisition Unit (DAQ). This device reads the voltages from the gages and converts them to strain as well as taking load cell data from the Instron. See Figure 6.3 for the setup of the computers, DAQ, and Instron. 


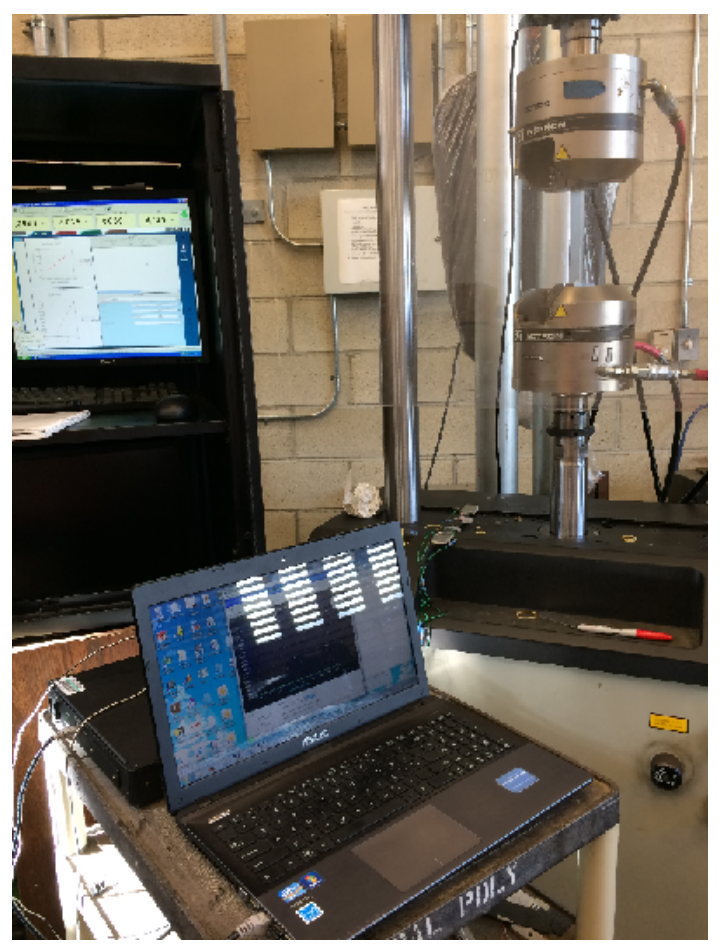

Figure 6.3: Computer Set-Up with Data Acquisition Unit and Instron

\subsubsection{Strain Gage Application}

For applications where high accuracy strain measurement is desired for a structure, strain gages may be used. In this research, strain gages were used to determine the Poisson's ratio for each composite material. See the Poisson's Ratio section above for more details. This section will focus on the preparation of the specimen for strain gage application. There are many different types of strain gages for various applications and requirements. The gages used in this research were the student version strain gages (CEA-06-240UZ-120), which were larger than the commercial ones.

The first step was to identify and sand the desired location of the strain gages. The area should be sanded with 400 grit sand paper to provide a smooth, even bonding surface. Avoid sanding into the fibers because that affects the measured properties. Next, the area must be cleaned with isopropyl alcohol. Then, a border was created with tape around the spot where the strain gage was to be bonded. 
The surface of the tape must be marked to provide markers for the alignment of the strain gage. The tape was used to prevent contamination of the bond area.

The gage should be taken out of its sleeve and placed on top of a sanitized, smooth surface such as the aluminum strip shown. Ensure that the shiny side of the strain gage is facing the top; otherwise, the gage may be glued upside down. Specialized tape is then applied on top of the gage with excessive tape on the ends. Carefully, tug on the tape at a shallow angle below $45^{\circ}$ to remove the gage. This step is crucial because the strain gages are very sensitive and easily damaged if bent.

Next, align the arrows on the strain gage to the markers made earlier. When satisfied with the alignment, pick up the gage from one side while leaving enough tape to act as a hinge. Next, brush a very light coat of catalyst on the back of the strain gage. If the blue dye is very apparent, too much catalyst has been applied. Add a small drop of the adhesive at the inner edge of the gage (towards the hinge) and apply the gage to the composite surface. Quickly spread the adhesive using constant pressure with a gauze pad. Lastly, apply constant pressure on the gage with your thumb for 90 seconds. When the adhesive is completely cured, the tape should come off cleanly. See Figure 6.4 for specimens with strain gages bonded.

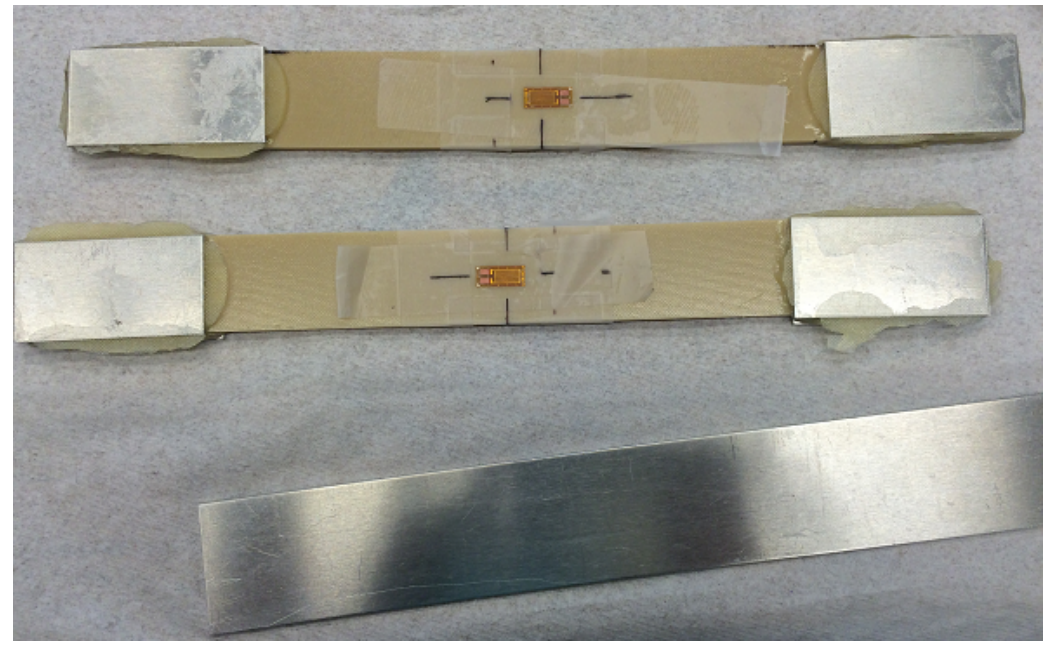

Figure 6.4: Test Specimens with Strain Gages Bonded 
The last step of preparing the strain gage for testing involves soldering the pads on the gage itself as well as the wires used to connect to the DAC. After soldering, flux remover must be applied 5 times around all areas to remove all remaining flux. The flux is helpful for the resin core to flow while soldering but it affects the measurement if not removed from the connection. Figure 6.5 below shows a specimen prepared for testing with a strain gage bonded and soldered to wires.

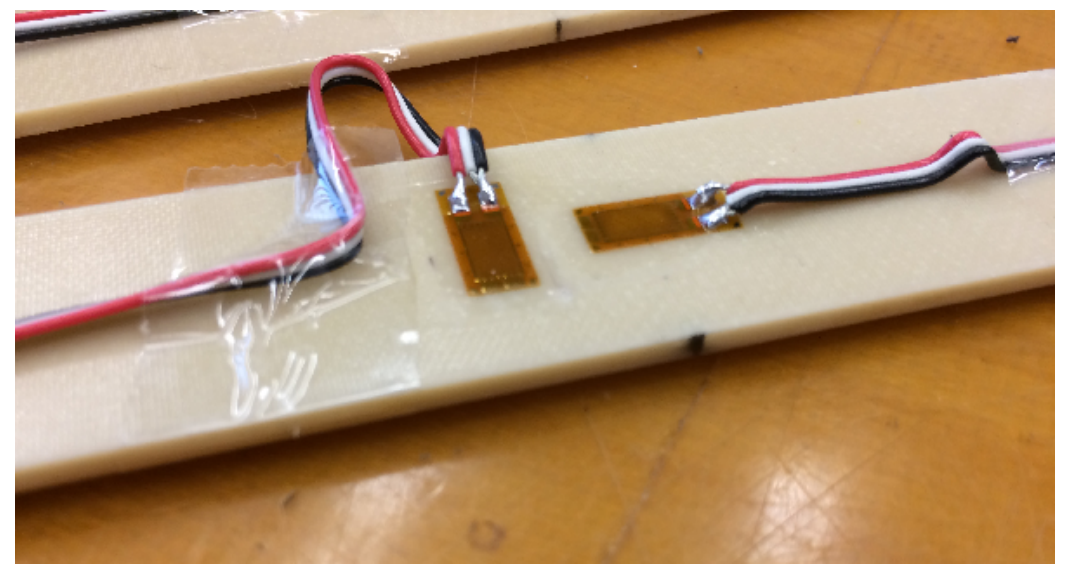

Figure 6.5: Strain Gage Bonded and Soldered to Wires

\subsubsection{Volume Fraction}

Fiber volume fraction describes the ratio of fibers to matrix in a composite material. To determine the fiber volume fraction, a sample of the composite must be measured before and after burning the resin. According to ASTM D2584, the resin should be burned at a temperature below $600^{\circ} \mathrm{C}$. The recommended sample size was 1 inch by 1 inch squares with a total mass of around 5 grams. These samples were burned in a small oven in the Composites Lab as shown in Figure 6.6 .

The mass measured post-burn is the mass of the fibers and the mass difference is the mass of the resin. The density of the resin and fibers can be found in the material datasheet. The fiber volume fraction is 


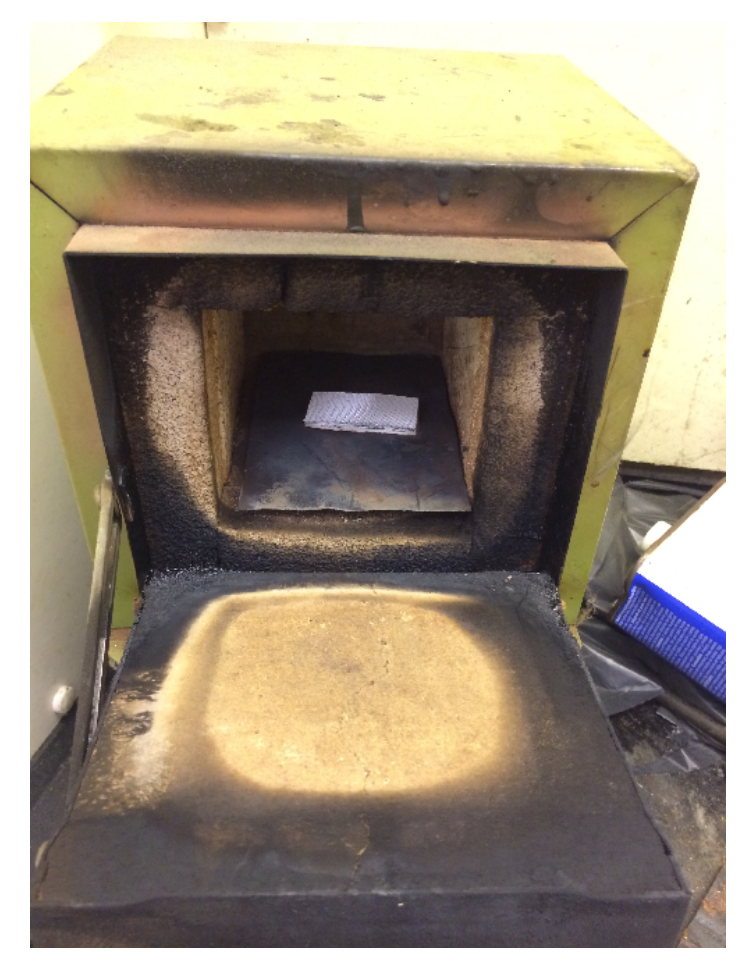

Figure 6.6: FG Material Burned in Oven for Volume Fraction Test

$$
V_{f}=\frac{m_{f}}{\left(m_{f}+\frac{m_{m}}{\rho_{m}} \rho_{f}\right)}
$$

Where $\rho_{f}$ is the density of the fiber, $\rho_{m}$ is the density of the matrix, and $m_{f}$ is the mass of the fiber, and $m_{m}$ is the mass of the matrix.

\subsection{Flat and Corrugated Specimen Test Procedure}

This section describes the experimental test procedures used in this research. The Instron machine was necessary to conduct the tension test and 3-point bend test. Both flat and corrugated specimens were tested under the same procedures. The only difference between the tensile tests was the lack of an extensometer during the corrugated transverse tension tests. The bend tests were conducted with the same test procedure for flat and corrugated specimens. 


\subsubsection{Uniaxial Tension Test}

This test was conducted to measure the load and deflection while load was applied in the axial direction. The extensional stiffness of the specimens was calculated from the stress and strain. There was no standard for testing corrugated composite structures, so the tension test was based on ASTM D3039. During the test, load was applied at 0.05 inches per minute until failure. As mentioned, all specimen types except for corrugated transverse specimens will have strain measurements by an extensometer. The extensometer was secured to the specimen using rubber bands as shown in Figure 6.7. After the extensometer was secured, the safety pin was removed and the strain balanced prior to the start of the test. For tests using the extensometer, the test was paused at $30 \%$ of the ultimate load. The load at which the test was paused must be manually set in the test case in the Bluehill2 software. The ultimate load was determined by testing one specimen of each type until failure.

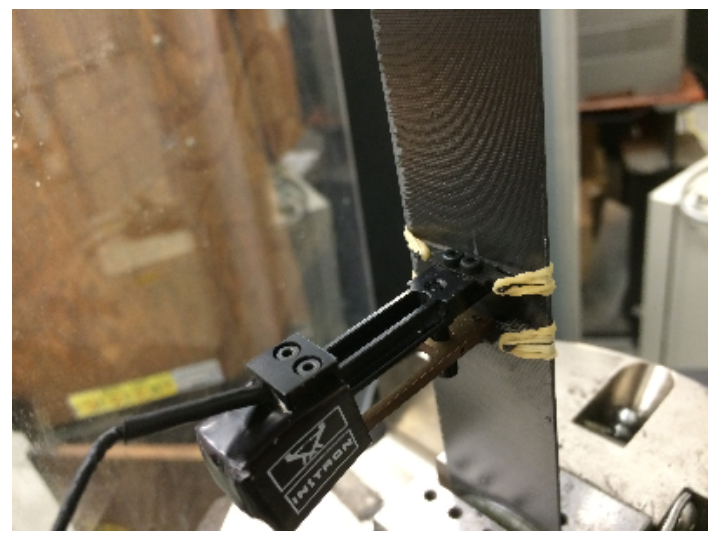

Figure 6.7: Extensometer Secured to a Test Specimen

While the test was paused, the extensometer was removed from the specimen. Then, the test continued until ultimate failure. The test was programmed in Bluehill2 to stop when the load dropped below $40 \%$ of the peak load. For tests without the use of the extensometer, the test ran until ultimate failure without a pause. However, some of the corrugated transverse specimen tests had to be manually 
stopped. The program did not recognize failure due to the small magnitude of the peak load for this specimen type.

\subsubsection{Three-Point Bend Test}

The bend test was used to determine the flexural stiffness and strength properties of a structure. The three-point bend test was used to determine the flexural properties of specimens in this research. Three-point bend tests involve a center loading on the specimen that is simply supported with two pins. The support pins and loading pin are identical and have 1-inch diameters. For this test, ASTM D7264 was the standard test method referenced for both flat and corrugated composites specimens because there is no official test standard for corrugated composites.

Prior to the start of the test, the locations of the pins were marked on the specimen. Each specimen was aligned to the loading pin and contact was made between all the pins and the specimen before the start of the test. A specimen aligned to the pins just prior to testing is shown in Figure 6.8.

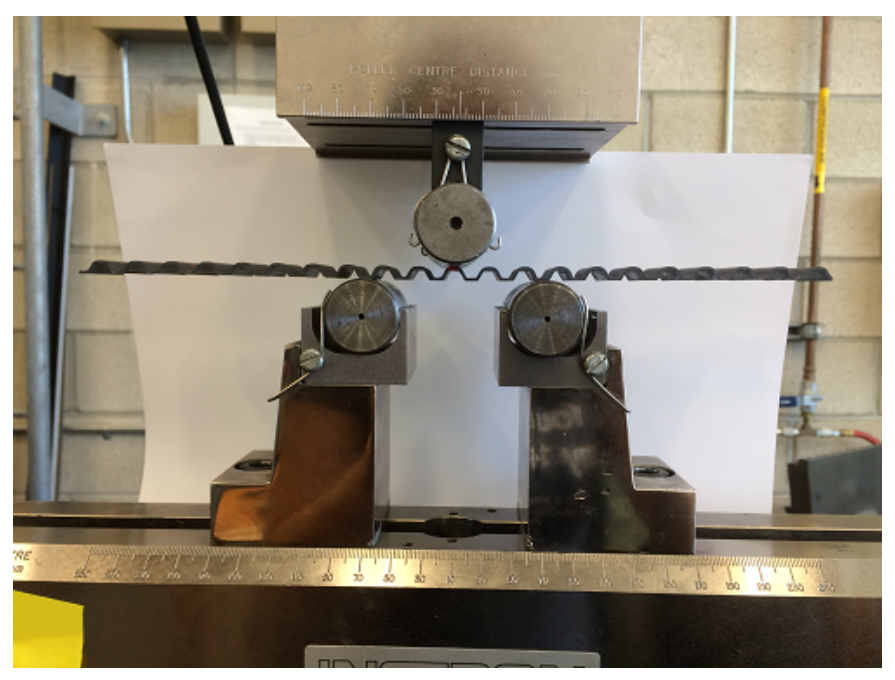

Figure 6.8: Corrugated CF Specimen Aligned for Three-Point Bend Test

During the test, load was applied at a rate of 0.05 inches per minute. The span length, which is the distance between the bottom support pins, was 2.38 inches 
for both flat and corrugated specimens. Generally, a high span length-to-thickness ratio is desired. This yielded span-to-thickness ratios of 59.5:1 and 54:1 for flat FG and CF specimens, respectively. The span-to-thickness ratio for corrugated FG and CF were 13.22:1 and 12.9:1, respectively.

The span length was chosen after preliminary testing to determine the length at which specimens could be tested for failure. From preliminary testing of corrugated specimens, it was determined that the support pins had to be centered on the lower flat regions of the trapezoids. Otherwise, the corrugated specimens slip. However, there was also a limit on how close the pins can be together. Due to the high flexibility characteristics of the specimens, the flat FG specimens did not fail before slipping on the support pins. Thus, maximum flexural strength could not be determined for flat FG specimens and the test was stopped before the specimens slipped on the support pins as shown in Figure 6.9. The test was stopped once a peak load was reached and the specimen failed.

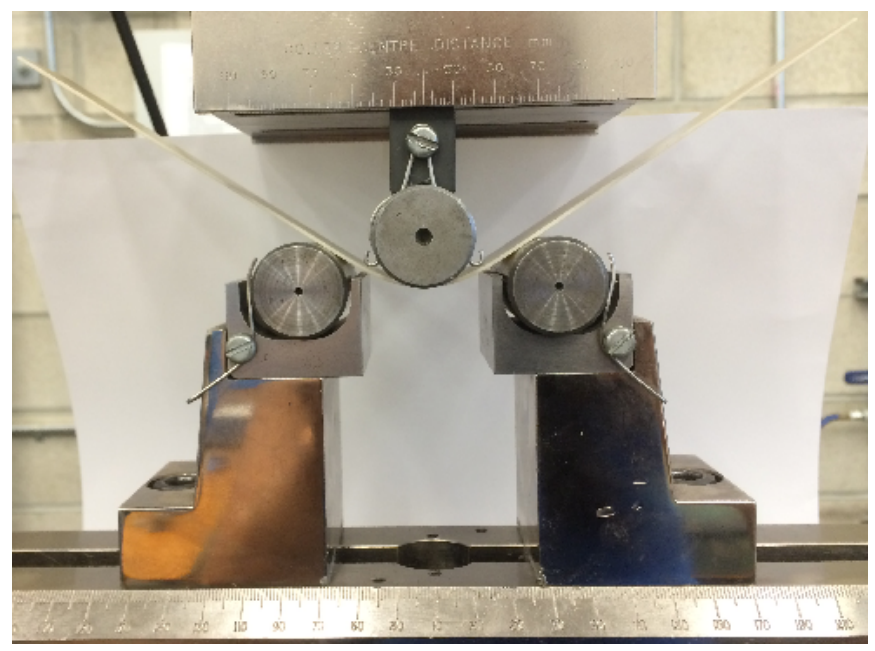

Figure 6.9: Specimen Slipping in Three-Point Bend Test 


\section{CHAPTER 7: EXPERIMENTAL RESULTS AND DISCUSSION}

The following chapter will discuss the experimental mechanical properties of the fiberglass material and results obtained experimentally from tensile and 3-point bend tests. The extensional and flexural stiffness of flat and corrugated specimens will be compared. Qualitative differences between the different composite specimens will be discussed as well. Finally, the experimental, analytical, and FEA results will be compared.

\subsection{Fiberglass Weave Mechanical Properties}

Tests were conducted to determine the material properties of Cytec MXB7701 FG. The Cytec FG was tested for material properties because this material was expired and donated to the lab a number of years ago. Thus, the material properties may have been affected by the storage conditions. The material may not have been sealed well or stored under the optimal temperature conditions. The NCT301 TR50S was also tested and properties were determined. Those results won't be mentioned in this paper because that material was not used for the experimental specimens due to issues with its manufacturability for the scope of this research. The Tencate TC250/M46J was not tested for material properties because another graduate student who used this material obtained the necessary data from the manufacturer. The test procedures for each type of test were described in Section 6.1. The results of those tests will be discussed in this section.

Material properties tests involved testing specimens in tension and compression until failure to determine the modulus and strength in each direction. An extensometer was attached to tensile test specimens as shown in Figure 7.1. The extensometer provides an accurate measurement of the displacement, so the tensile modulus was calculated using data from the extensometer. 


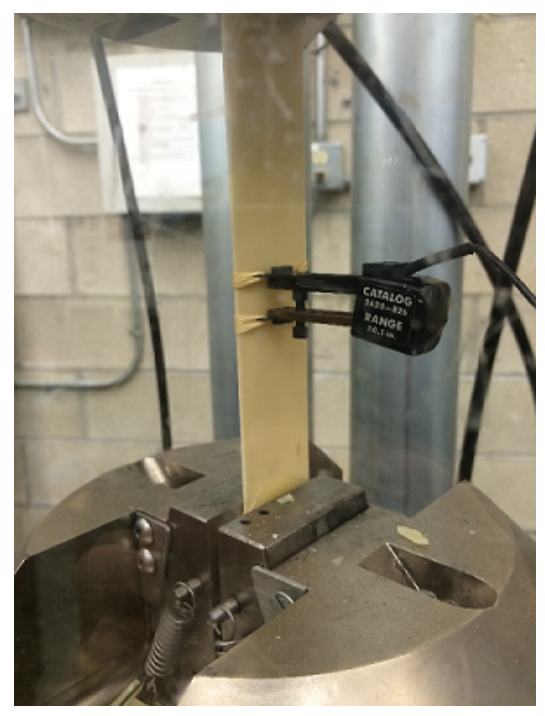

Figure 7.1: FG Material Test Specimen Measured with an Extensometer

The stress-strain curve of the FG material tests under tension and compression are shown in Figure 7.2. For the tension stress-strain plot, the initial linear region was data measured using an extensometer. Specimens tested under compression were not measured with an extensometer; the strain data was purely from the Instron cross-head displacement.

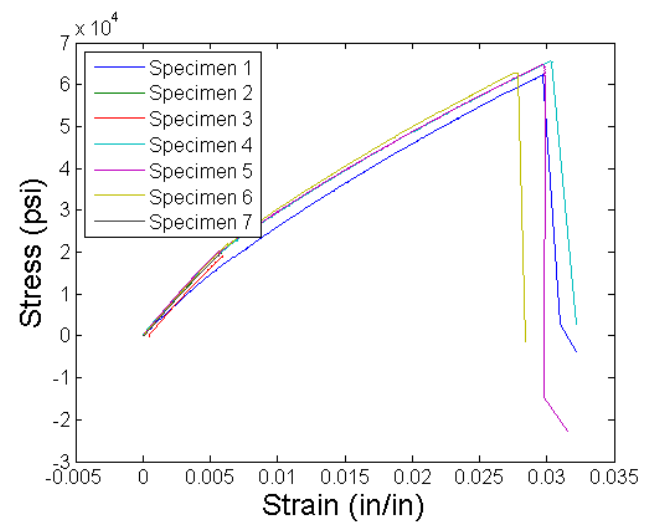

(a) Tension

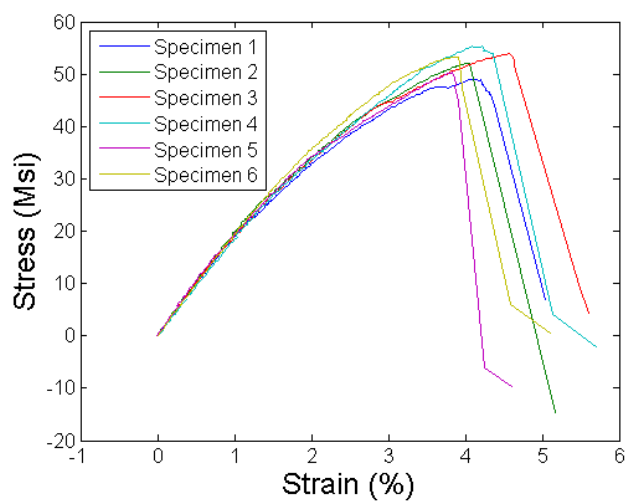

(b) Compression

Figure 7.2: Stress-Strain Curves of FG Material Test Specimens

The experimental moduli and strength in tension and compression are compared to values from the datasheet in Table 7.1 below. The experimental tensile properties appear to be very close to what was specified on the datasheet. However, 
the experimental compressive properties were significantly lower in comparison to the datasheet values. The difference may be attributed to the source of strain data because the Instron cross-head displacement was less accurate than data from the extensometer.

Table 7.1: Cytec MXB 7701/7781 Material Properties

\begin{tabular}{|c|c|c|}
\hline & Datasheet & Experimental \\
\hline $0^{\circ}$ Tensile Modulus (Msi) & $3.2-3.4$ & 3.5 \\
$0^{\circ}$ Tensile Strength (ksi) & $59-61$ & 64 \\
$0^{\circ}$ Compressive Modulus (Msi) & $3.8-4.0$ & 2.0 \\
$0^{\circ}$ Compressive Strength (ksi) & $64-69$ & 52 \\
Fiber Volume Fraction & $50-55 \%$ & $60.8 \%$ \\
\hline
\end{tabular}

The fiber volume fraction reveals the ratio of fibers to matrix in a composite material. According to the datasheet, the expected fiber volume fraction should be between 50-55\%. The fiber volume fraction obtained experimentally was $60.75 \%$, which is higher than the value specified in the datasheet. An increase in the fiber volume fraction means there should have been more resin in the composite material but some of that resin has been lost since the pre-preg material was prepared. This result was consistent with the higher tensile modulus compared to the datasheet value. Figure 7.3 shows the FG material before and after the resin was burned off. In Figure 7.3b, the resin was completely burned off and only the white FG fibers remained. Prior to the burn, the material was a tan color and post-burn, the remains were shiny, white FG fibers.

The Poisson's ratio was not listed in the datasheet and had to be determined experimentally using strain gages. The Poisson's ratio was calculated to be 0.12 from Equation 6.1. This result is reasonable for a woven composite material. 


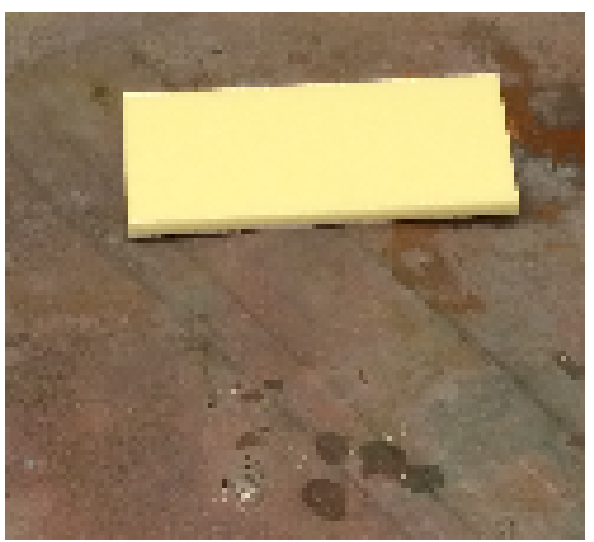

(a) Before Burn

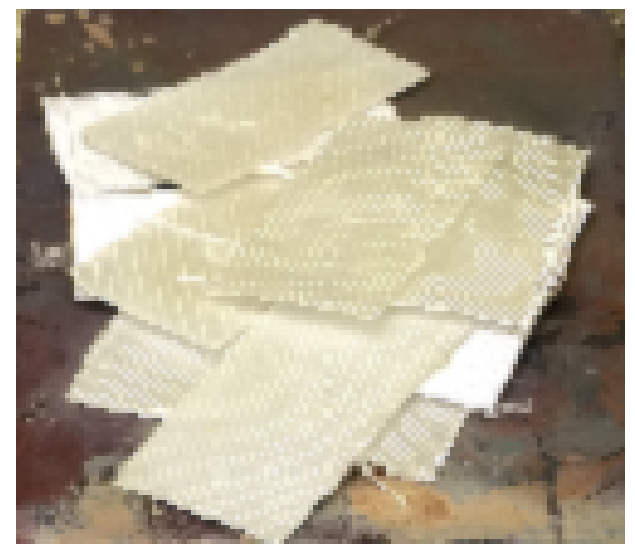

(b) After Burn

Figure 7.3: Pre- and Post-burn of FG Material for Volume Fraction Test

\subsection{Experimental Results}

This section will show the experimental results for both tension and bend tests. Flat and corrugated specimens were tested in tension and in a three-point bend test to determine their stiffness properties. The stiffness properties of flat and corrugated specimens were compared to observe the effect of corrugations. The results below are organized by test and material type.

Load and deflection data were measured during the test. Stress in tensile specimens was calculated from the data as

$$
\sigma=\frac{P}{A_{e f f}}
$$

where $P$ is the load in pounds and $A_{\text {eff }}$ is the effective cross section area in square inches. The effective area is defined as

$$
A_{\text {eff }}=w h
$$

$w$ is the width of the specimen, and $h$ is the height of the specimen. Extensional 
stiffness was calculated as

$$
E=\frac{\sigma}{\epsilon}
$$

where $\sigma$ is stress and $\epsilon$ is strain.

\subsubsection{Extensional Stiffness Results}

The extensional stiffness properties of the specimens were obtained from uniaxial tension tests. Refer to Section 6.2.1 for details about the test procedure. Stiffness was calculated from the linear portion of the stress-strain curve. For each test, corrugated specimens were selected from different layups to rule out inconsistencies introduced during the manufacturing process.

\section{Flat Tension Specimens}

Flat and corrugated fiberglass specimens were tested for the effects of corrugation on extensional stiffness properties. The gage area of both types of specimens contained the same amount of material.

The load-deformation plots for each specimen type are shown in Appendix B.1. The stress-strain curves of the flat specimens tested in tension are shown in Figure 7.4. Observing the linear region of the curve closely, there are two slopes. The deflection data measured in the first segment is from the extensometer, which was measured with higher accuracy. The displacement data following that point was measured from the cross-head displacement. Thus, the stiffness was calculated based on the initial linear region with measurement data from the extensometer.

The gage length of the specimens was determined such that the amount of composite material would be the same as the corrugated specimens. The transverse specimens failed before the longitudinal specimens for FG specimens. The longitudinal specimens held 1000 pounds more than the transverse specimens. This was expected due to the larger effective gage area of flat longitudinal FG specimens. 


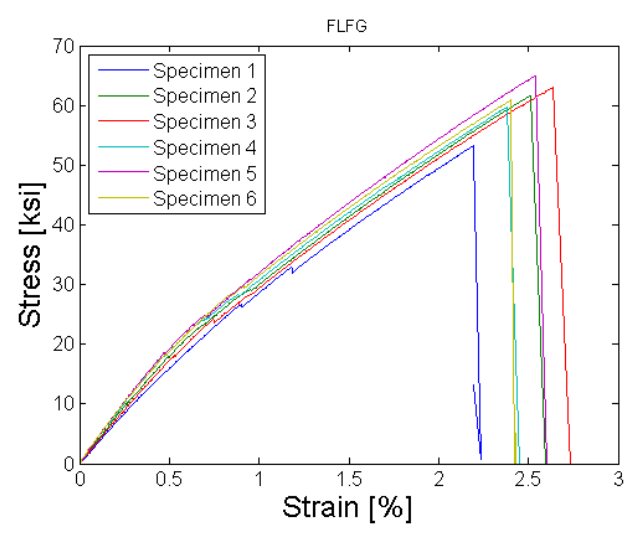

(a) Flat Longitudinal FG

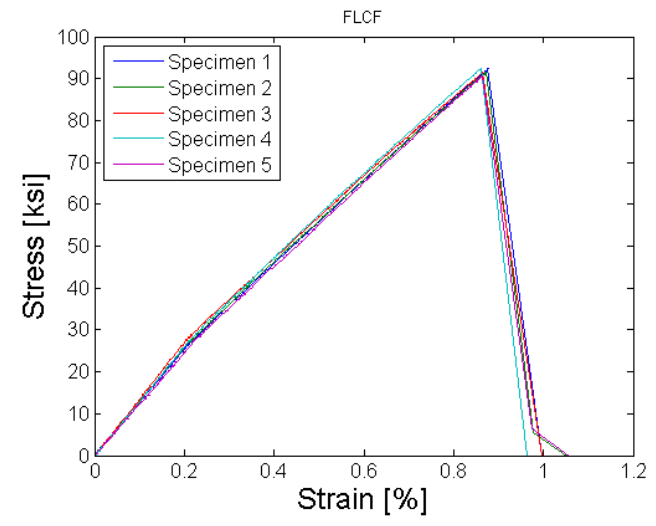

(c) Flat Longitudinal CF

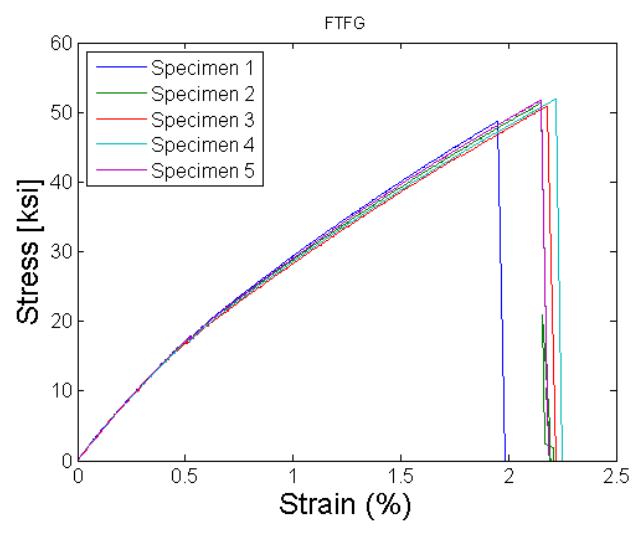

(b) Flat Transverse FG

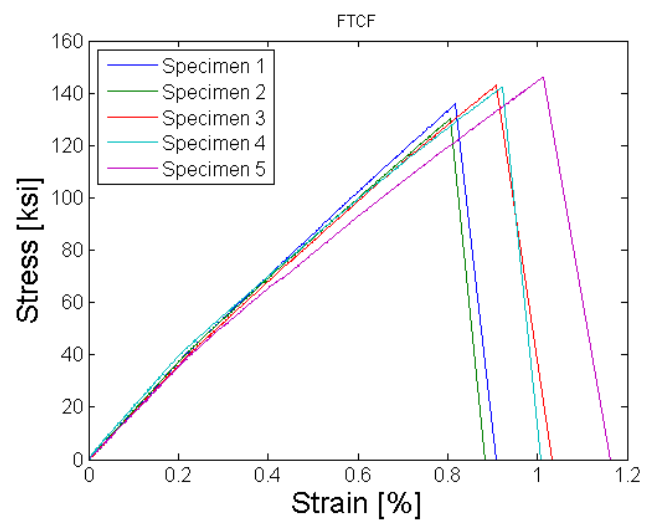

(d) Flat Transverse CF

Figure 7.4: Stress-Strain Curves of Flat Tensile Specimens

However, the opposite was true for the flat CF specimens. The transverse CF specimens held 1000 pounds more than the longitudinal specimens because $60 \%$ of the fibers were in the transverse direction. This layup sequence was designed to strengthen the corrugated composites in the transverse direction.

Stiffness was calculated using the first linear region of the curve as mentioned. There were at least 5 test specimens whose stress-strain curves matched well in the linear region. These specimens consistently failed around the same strength.

Between the two directions, the extensional stiffness of each flat composite is very close. The stiffness values and ultimate strength for each material and direction are shown in Table 7.2. The ratio of longitudinal stiffness to transverse stiffness is 1.0 and 0.7 for the flat FG and CF specimens, respectively. 
Table 7.2: Tensile Properties of Flat Test Specimens

\begin{tabular}{|c|c|c|c|}
\hline Material & Direction & Extensional Stiffness [Msi] & Ultimate Strength [ksi] \\
\hline FG & Longitudinal & 3.722 & 62.040 \\
FG & Transverse & 3.496 & 51.177 \\
CF & Longitudinal & 12.879 & 91.682 \\
CF & Transverse & 18.543 & 138.07 \\
\hline
\end{tabular}

The flat specimens were tested as a control for the composite layups in order to observe changes due to corrugations in weave and cross-ply composites. Only the maximum strength and load were compared to the corrugated composites tested in tension in the next section.

\section{Corrugated Tension Specimens}

Corrugated composites were expected to have very different stiffnesses depending on the direction with respect to the corrugations. The stiffness was expected to be greater in the longitudinal direction. These specimens were manufactured and tested in uniaxial tension tests. The load-deformation plots are shown in Appendix B.2.

Stress-strain curves for the corrugated specimens under tensile load are shown in Figure 7.5. The curves for the transverse specimens appear to have more noise in the data than that of the longitudinal specimens. The strain data for corrugated longitudinal specimens was captured with an extensometer while the strain data was taken from the Instron cross-head displacement for corrugated transverse specimens. Transverse specimens were more flexible, which led to large deformations under low loads.

Failure modes of the corrugated composites were lateral and delamination. Longitudinal specimens failed laterally in the gage area similar to the flat specimens. These composites exhibit the same failure modes as brittle materials, where ultimate yield occurs without plastic deformation. However, the transverse spec- 


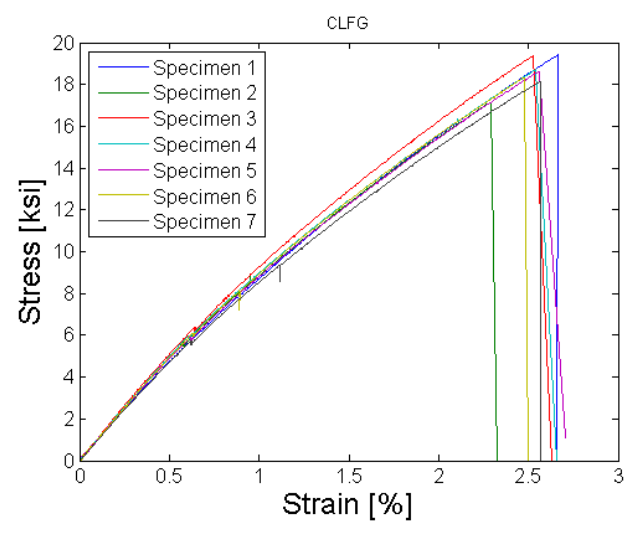

(a) Corrugated Longitudinal FG

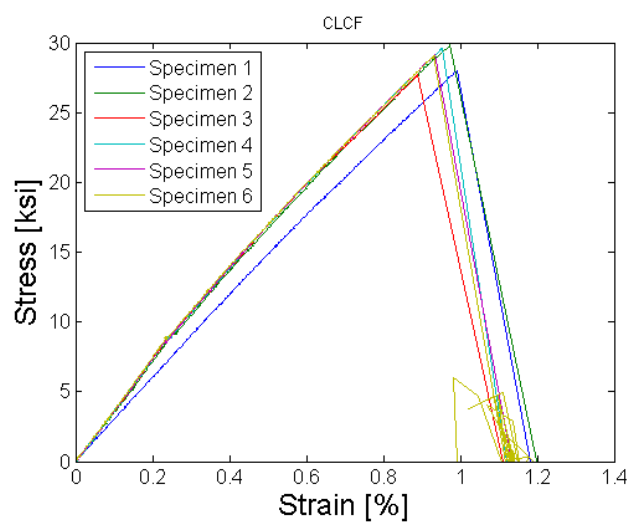

(c) Corrugated Longitudinal CF

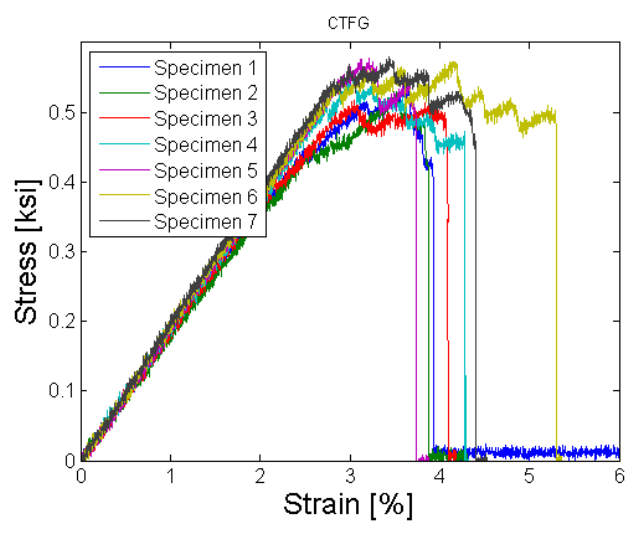

(b) Corrugated Transverse FG

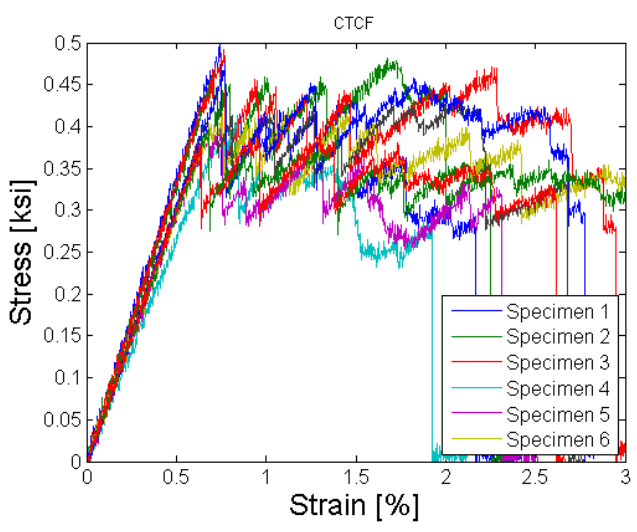

(d) Corrugated Transverse CF

Figure 7.5: Stress-Strain Curves of Corrugated Tensile Specimens

imens initially fail due to delamination of the plies. This resulted in the first dip of the curve ending the linear region and marking the start of plastic deformation. The plastic region includes a series of peaks and valleys.

The peaks and valleys represent the local buckling of the corrugations as the specimen stretched. While the specimen undergoes plastic deformation, it was able to recover from the small delamination and local buckling to continue sustaining load. The fibers continued to sustain load until a final hinge failure. The transition from linearity to plasticity was smoother for the transverse FG specimens than the transverse $\mathrm{CF}$ specimens. Meanwhile, the $\mathrm{CF}$ specimens had a larger ratio of plastic to elastic deformation than the FG specimens until ultimate hinge failure.

Extensional stiffness was calculated based on the linear portion of the stress- 
strain curves. Refer to Table 7.3 for structural properties of corrugated composites under tensile load. The stiffnesses of flat and corrugated composites can't be compared side-by-side due to the difference in the effective cross-sectional area. The effective cross-sectional areas of the corrugated composites are much larger than the flat composites due to its geometry. The effects of corrugation on the composites can be captured by the difference in the ratio of longitudinal stiffness to transverse stiffness. The extensional stiffness ratio for corrugated FG and CF specimens are 49.3 and 61.3 , respectively.

Table 7.3: Tensile Properties of Corrugated Test Specimens

\begin{tabular}{|c|c|c|c|}
\hline Material & Direction & Extensional Stiffness [Msi] & Ultimate Strength [ksi] \\
\hline FG & Longitudinal & 0.94256 & 18.529 \\
FG & Transverse & 0.019124 & 0.5148 \\
CF & Longitudinal & 3.701600 & 28.90 \\
CF & Transverse & 0.060418 & 0.4372 \\
\hline
\end{tabular}

\subsubsection{Flexural Stiffness Results}

Flexural properties of the flat and corrugated composites were obtained by testing specimens in a three-point bend test. From the load and deflection data, the flexural stiffnesses were calculated. High flexural stiffness is a desired property for applications where it may be subject to flexural loads such as sandwich plates or bending loads on aircraft wings.

Flexural stiffness in the longitudinal direction of a corrugated coupon is calculated as

$$
D_{\text {long }}=\frac{E I}{\frac{h}{2}}
$$

where $I$ is the area moment of inertia, $h$ is the height of the coupon, and $E$ is defined as

$$
E=\frac{L^{3} m}{4 b h^{3}}
$$


where $L$ is the length of the coupon, $m$ is the slope of the load-deformation curve, and $b$ and $h$ is the width and height of the coupon, respectively.

Flexural stiffness for all other coupon types is calculated as

$$
D_{\text {trans }}=\frac{E h^{3}}{12} .
$$

\section{Flat Flexure Specimens}

The FG specimens were only tested for results in the linear region because the specimens were too flexible. The specimens slipped from the rollers before failure could occur. Meanwhile, CF specimens were stiffer and tested until failure. Stiffness properties of these flat specimens were calculated from the load-deformation curves in Appendix B.3 below.

From the load-deformation curves, the slopes were determined from curvefitting. The average slopes were determined to be 81.05 and 71.42 pounds per inch for the longitudinal and transverse flat FG specimens, respectively. The average slopes were 202.3 and 560.5 pounds per inch for the longitudinal and transverse flat CF specimens, respectively. Stress-strain curves are shown in Figure 7.6.

Similar to the tensile test, the flat $\mathrm{CF}$ specimens were stiffer in the transverse direction than in the longitudinal direction. The flat transverse CF specimens were stiffer because there were more fibers in the transverse direction. The flexural stiffness properties are listed in Table 7.4 below. The longitudinal to transverse flexural stiffness ratios are 0.95 and 0.30 for FG and CF specimens, respectively. As expected, the ratio for flat FG specimens was close to 1 because the FG material was a satin weave. 


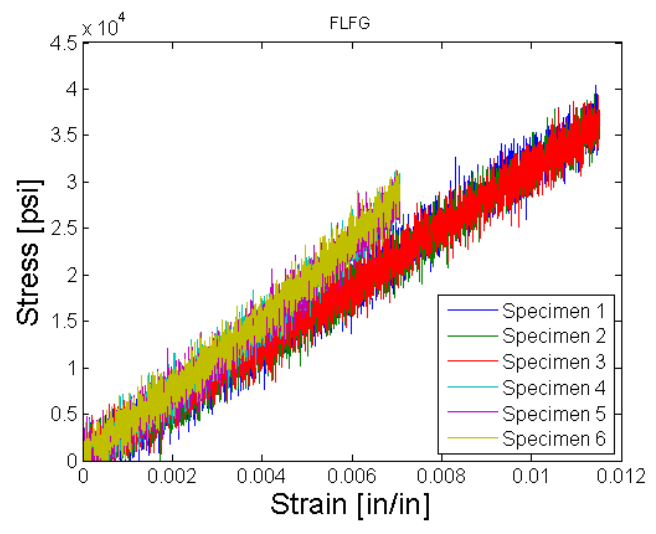

(a) Flat Longitudinal FG

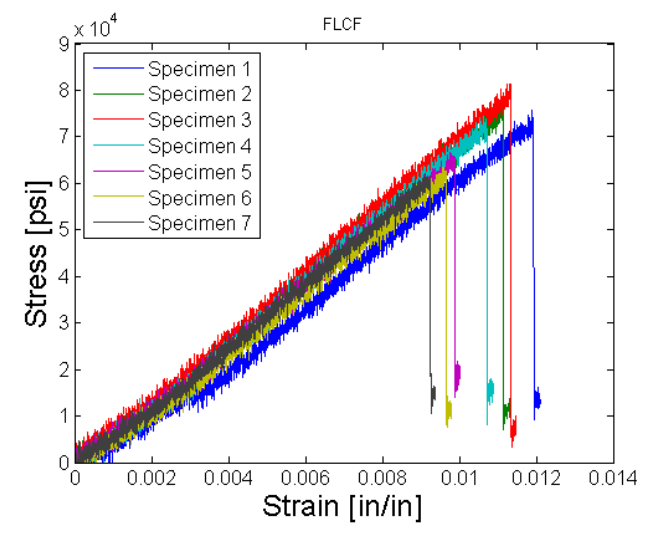

(c) Flat Longitudinal CF

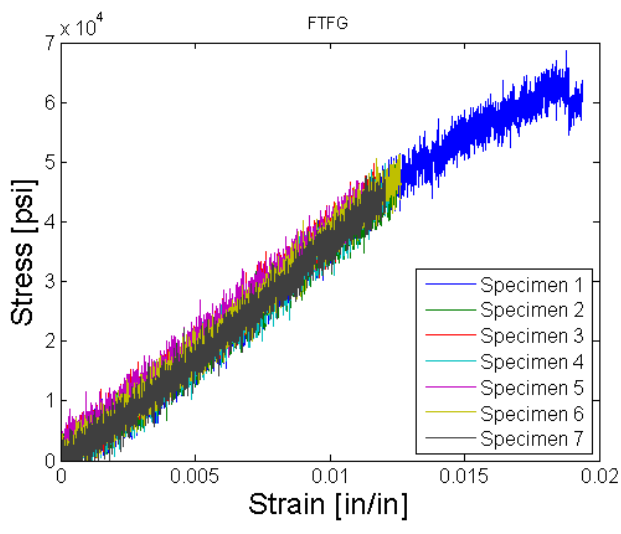

(b) Flat Transverse FG

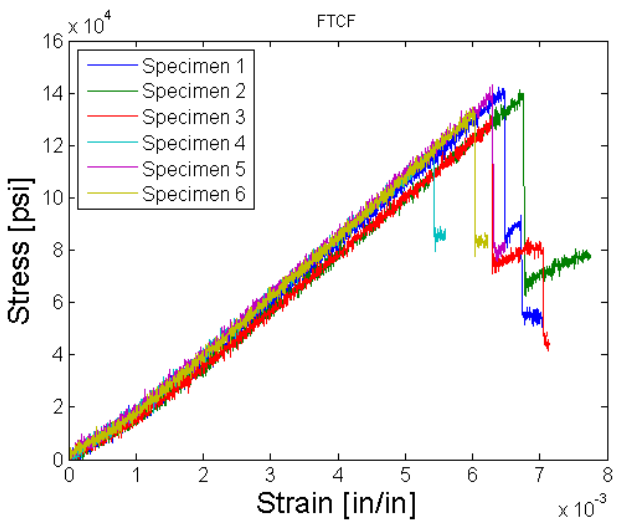

(d) Flat Transverse CF

Figure 7.6: Stress-Strain Curves of Flat Flexure Specimens

\section{Corrugated Flexure Specimens}

Corrugated specimens were tested in bending to determine its flexural properties.

These specimens were tested with the same procedure as the flat specimens in the previous section. Load and displacement data were collected from the Instron load cells. Load-deformation curves are shown in Appendix B.4. Stress-strain plots are shown in Figure 7.7.

As shown in the plots, the maximum load for the longitudinal FG specimens was far greater than that of the transverse FG specimens. The plots for all the transverse specimens had a significant amount of noise because very small loads were measured. There was only one functioning load cell at the time and I neglected to check the tolerance of the load cell until after testing. The applied loads 
Table 7.4: Flexural Properties of Flat Test Specimens

\begin{tabular}{|c|c|c|c|}
\hline Material & Direction & Stiffness $D\left[\frac{\left[b_{f}\right.}{i n}\right]$ & Ultimate Strength $[\mathrm{ksi}]$ \\
\hline FG & Longitudinal & 18.95 & - \\
FG & Transverse & 19.93 & - \\
CF & Longitudinal & 47.61 & 71.056 \\
CF & Transverse & 156.18 & 135.500 \\
\hline
\end{tabular}

for the transverse bend tests were so small that it was within the measurement error of the load cell. This explains the noise in the measured load data. The corrugated specimens primarily failed from delamination and tension. The end of the linear regions in the plots for transverse specimens signaled the start of delamination.

After the first linear region, there were peaks and valleys in the curve. As with the corrugated specimens under tensile load, the peaks and valleys in the curve were due to local buckling and delamination. The fibers that remained intact continued to take load post-delamination. The stress increased until the corrugated specimens were fractured at the hinge. Refer to Table 7.5 for flexural stiffness and strength characteristics.

Table 7.5: Flexural Properties of Corrugated Test Specimens

\begin{tabular}{|c|c|c|c|}
\hline Material & Direction & Stiffness $D\left[\mathrm{in} \cdot l b_{f}\right]$ & Ultimate Strength $[\mathrm{ksi}]$ \\
\hline FG & Longitudinal & 1919.7 & 18.750 \\
FG & Transverse & 10.26 & 1.5934 \\
CF & Longitudinal & 4620.6 & 16.855 \\
CF & Transverse & 56.79 & 1.0619 \\
\hline
\end{tabular}

The flexural stiffnesses for the longitudinal specimens are much higher than the transverse specimens. The CF specimens were stiffer than the FG specimens in both directions. While the CF coupons were stiffer in bending, the corrugations had a greater impact on the FG coupons. The longitudinal to transverse stiffness ratio was 187.1 and 81.4 for FG and CF specimens, respectively. 


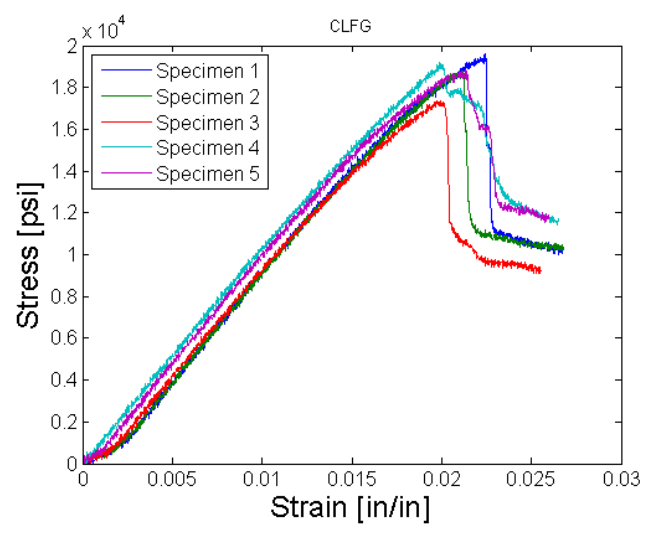

(a) Corrugated Longitudinal FG

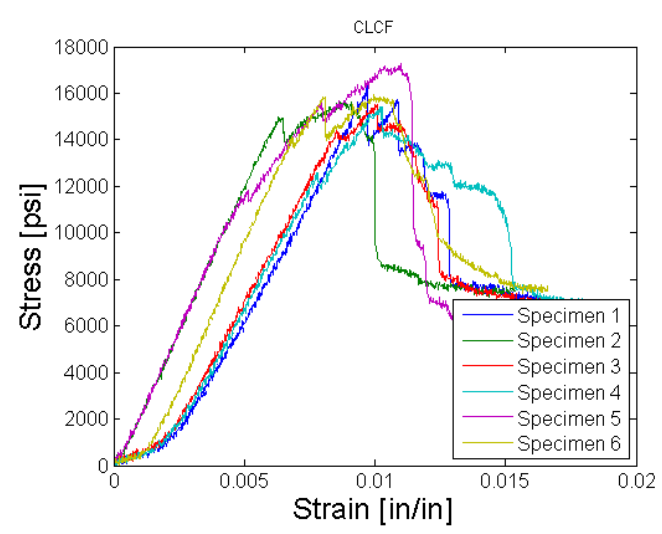

(c) Corrugated Longitudinal CF

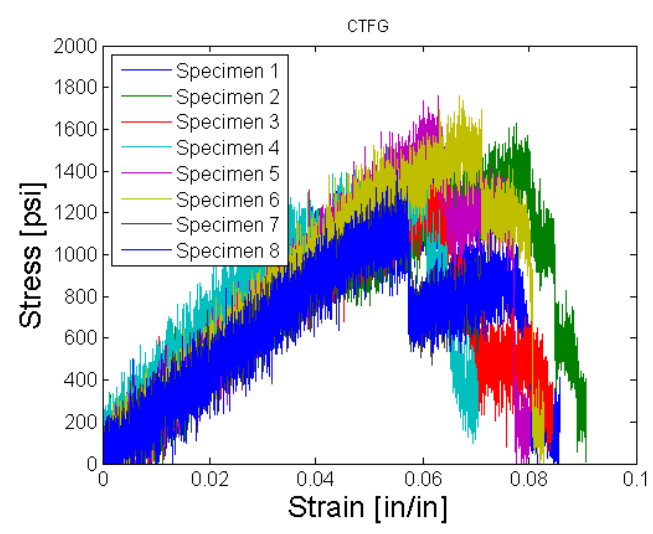

(b) Corrugated Transverse FG

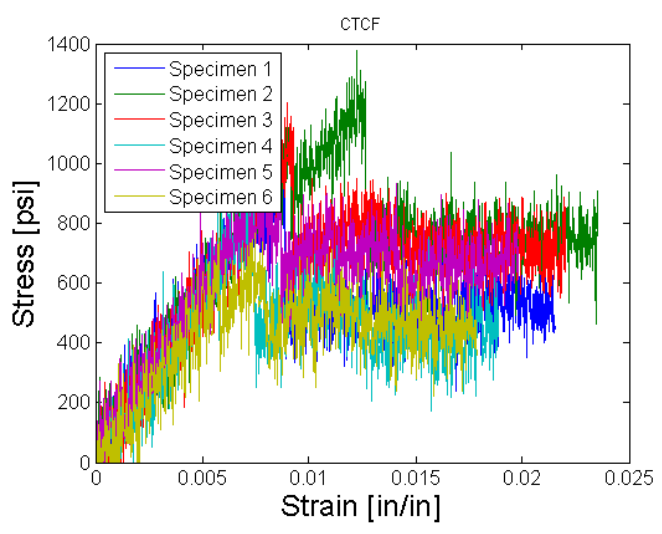

(d) Corrugated Transverse CF

Figure 7.7: Stress-Strain Curves of Corrugated Flexure Specimens

7.3 Experimental, Numerical, and Analytical Results and Discussion

This section will discuss the extensional and flexural stiffnesses of each specimen type obtained experimentally. The stiffness ratios of flat and corrugated specimens will be compared to observe changes due to the corrugations. Lastly, experimental results will be compared with the analytical and FEA results. This confirms whether the analytical and FEA models are good predictions of the stiffness properties of corrugated composites.

\subsubsection{Comparison of Flat Specimen Stiffness}

The extensional stiffness was determined experimentally from uniaxial tension tests. The test coupons were dimensioned to have approximately the same amount 
of composite material as its corrugated counterparts. First, the experimental results will be discussed. The extensional stiffness of flat specimens was calculated experimentally, analytically, and numerically. These values can be found in Table 7.6. The extensional stiffness of the FG specimens in both directions were about the same. This was expected from a satin weave material. The extensional stiffness ratio is the ratio of the longitudinal to transverse extensional stiffness. The extensional stiffness ratios for flat FG and CF composites were 1.0 and 0.7 , respectively.

Table 7.6: Comparison of Extensional Stiffness of Experimental, Analytical, and FEA Flat Test Specimens

\begin{tabular}{|c|c|c|c|c|}
\hline Material & Direction & Experimental [Msi] & Analytical [Msi] & FEA [Msi] \\
\hline FG & Longitudinal & 3.72 & 3.51 & 3.54 \\
FG & Transverse & 3.50 & 3.51 & 3.54 \\
CF & Longitudinal & 12.9 & 12.9 & 13.3 \\
CF & Transverse & 18.5 & 19.0 & 18.7 \\
\hline
\end{tabular}

Extensional stiffnesses calculated through experimental, analytical, and numerical methods were compared in Table 7.7. The maximum percentage difference between the analytical and experimental extensional stiffness was 5.74\%. And the maximum percentage difference between the FEA result and experimental is $4.89 \%$ for the flat longitudinal FG specimen. The flat specimens were tested as a control in this research because flat specimens have a simple geometry. Both analytical and FEA models for flat tensile specimens resulted in accurate predictions.

Table 7.7: Percentage Difference of Extensional Stiffness Between Experimental, Analytical and FEA Flat Test Specimens

\begin{tabular}{|c|c|c|c|c|}
\hline Material & Direction & Analytical & FEA & Anal/FEA \\
\hline FG & Longitudinal & 5.74 & 4.89 & 0.85 \\
FG & Transverse & 0.53 & 1.38 & 0.85 \\
CF & Longitudinal & 0.22 & 2.90 & 2.68 \\
CF & Transverse & 2.23 & 1.03 & 1.20 \\
\hline
\end{tabular}

Flat specimens were tested in three-point bend tests to determine the flexural 
stiffnesses. Flexural stiffness of each flat specimen was calculated using experimental, analytical, and numerical methods. These values are listed in Table 7.8. The flexural stiffness of FG specimens was similar in both directions as expected from a weave material. However, flat $\mathrm{CF}$ specimens in the transverse direction were three times stiffer than in the longitudinal direction. This was due to 3 plies in the transverse direction compared to 2 plies in the longitudinal direction. In a bend test, fibers in the transverse direction were able to carry higher load. The flexural stiffness ratio for flat FG and CF composites were 0.95 and 0.30 , respectively.

Table 7.8: Comparison of Experimental, Analytical, and FEA Flexural Stiffness of Flat Test Specimens

\begin{tabular}{|c|c|c|c|c|}
\hline Material & Direction & $\begin{array}{c}\text { Experimental } \\
{\left[\mathrm{in} \cdot l b_{f}\right]}\end{array}$ & $\begin{array}{c}\text { Analytical } \\
{\left[\text { in } \cdot l b_{f}\right]}\end{array}$ & $\begin{array}{c}\text { FEA } \\
{\left[\text { in } \cdot l b_{f}\right]}\end{array}$ \\
\hline FG & Longitudinal & 18.96 & 18.97 & 23.28 \\
FG & Transverse & 19.93 & 18.97 & 23.78 \\
CF & Longitudinal & 47.61 & 55.46 & 51.77 \\
CF & Transverse & 156.2 & 193.0 & 186.2 \\
\hline
\end{tabular}

The experimental results were compared with the analytical and FEA results in Table 7.9. Flexural stiffnesses calculated analytically for flat FG specimens were good predictions at a maximum difference of $4.95 \%$. Meanwhile, the flexural stiffnesses calculated analytically for flat CF specimens had a maximum difference of $20.1 \%$. The flexural stiffness determined from FEA had a maximum difference of $20.5 \%$ from the experimental results for the flat longitudinal FG specimen. Results from the analytical method and FEA were compared and the predicted flexural stiffnesses for flat CF specimens matched with less than $10 \%$ difference. Both analytical and FEA methods predicted higher stiffnesses.

\subsubsection{Comparison of Corrugated Specimen Stiffness}

The extensional stiffness of corrugated composites was determined from experimentation, analytical methods, and finite element analysis. From uniaxial tension 
Table 7.9: Percentage Difference of Flexural Stiffness Between Experimental, Analytical and FEA Flat Test Specimens

\begin{tabular}{|c|c|c|c|c|}
\hline Material & Direction & Analytical & FEA & Anal/FEA \\
\hline FG & Longitudinal & 0.00 & 20.5 & 20.4 \\
FG & Transverse & 4.95 & 17.6 & 22.5 \\
CF & Longitudinal & 15.2 & 8.36 & 6.88 \\
CF & Transverse & 21.1 & 17.5 & 3.59 \\
\hline
\end{tabular}

tests, the stress-strain curve was created for each specimen type from experimental data. The extensional stiffnesses in both longitudinal and transverse directions of corrugated composites are located in Table 7.10. The extensional stiffness ratio of corrugated FG and CF composites were 49.3 and 61.3, respectively. Comparing the extensional stiffness ratio of flat to corrugated composites, the stiffness ratio increased by 49.3 times and 87.6 times for FG and $\mathrm{CF}$, respectively.

Table 7.10: Comparison of Extensional Stiffness of Experimental, Analytical, and FEA Corrugated Test Specimens

\begin{tabular}{|c|c|c|c|c|}
\hline Material & Direction & Experimental [ksi] & Analytical [ksi] & FEA [ksi] \\
\hline FG & Longitudinal & 942.6 & 883.5 & 993.5 \\
FG & Transverse & 19.12 & 11.43 & 20.15 \\
CF & Longitudinal & 3702 & 3753 & 4424 \\
CF & Transverse & 60.42 & 49.6 & 41.6 \\
\hline
\end{tabular}

Extensional stiffnesses of corrugated composites calculated using analytical methods and FEA were compared with experimental results in Table 7.11. The analytical result was calculated using Golzar and Friswell for longitudinal and transverse directions, respectively, for both material types. For extensional stiffness, Golzar appeared to give a closer prediction. The analytical and experimental results differed at a maximum of $50.4 \%$, which was for the corrugated transverse FG specimen. The analytical method was able to accurately predict the stiffness of longitudinal corrugated composites with percentage differences lower than $10 \%$ for both FG and CF materials.

The maximum difference between FEA and experimental extensional stiffness 
Table 7.11: Percentage Difference of Extensional Stiffness Between Experimental, Analytical and FEA Corrugated Test Specimens

\begin{tabular}{|c|c|c|c|c|}
\hline Material & Direction & Analytical & FEA & Anal/FEA \\
\hline FG & Longitudinal & 6.5 & 10.4 & 16.8 \\
FG & Transverse & 50.4 & 5.2 & 55.3 \\
CF & Longitudinal & 1.4 & 17.8 & 16.4 \\
CF & Transverse & 19.7 & 36.9 & 17.5 \\
\hline
\end{tabular}

results was $36.9 \%$ for transverse CF. Results differed at a maximum of $55.3 \%$ and minimum of $16.4 \%$ between analytical and FEA results. The analytical method appears to have provided a better approximation in all cases except for corrugated transverse FG.

Flexural stiffness was calculated based on the three methods. The analytical and finite element results were compared with experimental results in Table 7.12. The flexural stiffness ratio of longitudinal to transverse for corrugated FG and CF specimens were 187.1 and 81.4, respectively. Compared to the flexural stiffness ratio of flat specimens, the corrugated flexural stiffness ratio was 196.9 and 271.3 times greater.

Table 7.12: Comparison of Flexural Stiffness of Experimental, Analytical, and FEA Corrugated Test Specimens

\begin{tabular}{|c|c|c|c|c|}
\hline Material & Direction & $\begin{array}{c}\text { Experimental } \\
{\left[i n \cdot l b_{f}\right]}\end{array}$ & $\begin{array}{c}\text { Analytical } \\
{\left[i n \cdot l b_{f}\right]}\end{array}$ & $\begin{array}{c}\text { FEA } \\
{\left[i n \cdot l b_{f}\right]}\end{array}$ \\
\hline FG & Longitudinal & 1920 & 1969 & 1742 \\
FG & Transverse & 10.26 & 13.20 & 13.24 \\
CF & Longitudinal & 4621 & 4710 & 8604 \\
CF & Transverse & 56.79 & 40.45 & 52.09 \\
\hline
\end{tabular}

The flexural stiffnesses calculated with different methods were compared to the flexural stiffness determined experimentally. Percentage differences between the experimental, analytical, and numerical methods are shown in Table 7.13. The analytical method was able to accurately predict flexural stiffness in longitudinal corrugated specimens but not in the transverse direction for both FG and CF. The maximum difference in the longitudinal direction was $2.54 \%$, which was for 
corrugated FG. The maximum difference in the transverse direction was $33.6 \%$, which was for corrugated $\mathrm{CF}$.

Table 7.13: Percentage Difference of Flexural Stiffness Between Experimental, Analytical, and FEA Corrugated Test Specimens

\begin{tabular}{|c|c|c|c|c|}
\hline Material & Direction & Analytical & FEA & Anal/FEA \\
\hline FG & Longitudinal & 2.54 & 9.70 & 12.2 \\
FG & Transverse & 25.1 & 25.4 & 0.3 \\
CF & Longitudinal & 1.92 & 60.2 & 58.5 \\
CF & Transverse & 33.6 & 8.65 & 25.2 \\
\hline
\end{tabular}

Predicted flexural stiffnesses were calculated from FEA and compared with experimental and analytical results. Predictions of flexural stiffness from FEA were inaccurate for longitudinal corrugated $\mathrm{CF}$, which had a maximum difference of $60.2 \%$. The flexural stiffnesses were accurately calculated from FEA for longitudinal corrugated FG and transverse corrugated CF, which were both within $10 \%$ difference from experimental flexural stiffnesses.

Calculated flexural stiffnesses from analytical methods and FEA were compared. The results for corrugated FG were close between the two methods. However, the results were varied for corrugated CF because one method resulted in a better prediction for the opposite direction.

The extensional and flexural stiffness ratios are summarized in Table 7.14 and 7.15 .

Table 7.14: Extensional Stiffness Ratios Determined Experimentally

\begin{tabular}{|c|c|c|}
\hline Material & Flat & Corrugated \\
\hline FG & 1.0 & 49.3 \\
CF & 0.7 & 61.3 \\
\hline
\end{tabular}

Table 7.15: Flexural Stiffness Ratios Determined Experimentally

\begin{tabular}{|c|c|c|}
\hline Material & Flat & Corrugated \\
\hline FG & 0.95 & 187.1 \\
CF & 0.3 & 81.4 \\
\hline
\end{tabular}




\section{CHAPTER 8: CONCLUSION}

The main goal of this research was to identify effects of corrugation on the stiffness characteristics of composite materials. In order to observe the effects, flat and corrugated composite coupons were manufactured and tested under the same

procedures. The corrugation effects were also compared between FG weave and cross-ply CF.

Extensional and flexural stiffnesses of the composites were determined experimentally from tension and three-point bend tests. The corrugation effects were measured by the change in stiffness ratio. The stiffness ratio was the ratio of stiffness in the longitudinal direction to stiffness in the transverse direction.

For FG coupons, the extensional stiffness ratio increased by 49.3 times with corrugations. The flexural stiffness ratio increased 196.9 times with corrugations introduced. Meanwhile the extensional stiffness ratio increased by 61.3 and the flexural stiffness ratio increased by 271.3 times in corrugated CF coupons. The change in the stiffness ratios were due to a combination of lower stiffness in the transverse direction and higher stiffness in the longitudinal direction.

The tensile ultimate load of flat and corrugated composite specimens were the same in the longitudinal direction. Meanwhile, the tensile ultimate load of corrugated composites was greatly reduced in the transverse direction. Thus, corrugations had a larger impact in the transverse direction under tensile load for both materials. Corrugations had a greater impact on the stiffness properties of cross-ply CF. The extensional and flexural stiffness ratios were higher in the cross-ply CF. 


\subsection{Future Recommendations}

This topic on stiffness characteristics of corrugated composites can be researched more thoroughly in many ways. The first major improvement in the manufacturing process is to machine a second mold. By curing the composite between two molds, the material should better conform to the shape of the corrugations as well as ease the layup process. Experimental results would be more accurate if the force data was collected from a load cell with higher accuracy for small loads seen by transverse coupons.

A layup sequence with angle plies should be easier to manufacture. A different ply layup such as plies in the $+45 /-45$-degree orientations may be explored to observe the effects on stiffness. There is currently no ASTM standard for the stiffness testing of corrugated composites. Research may be conducted to determine recommended coupon sizes for tension and flexure tests. 


\section{BIBLIOGRAPHY}

[1] Adam Mornement and Simon Holloway. "Corrugated Iron: Building on the Frontier". In: (2007), p. 10.

[2] Tomohiro Yokozeki et al. "Mechanical properties of corrugated composites for candidate materials of flexible wing structures". In: Composites: Part A applied science and manufacturing 37 (2006), pp. 1578-1586.

[3] Christophe Thill et al. "Investigation of trapezoidal corrugated aramid/ epoxy laminates under large tensile displacements transverse to the corrugation direction". In: Composites: Part A 41 (2010), pp. 168-176.

[4] Iman Dayyani, Saeed Ziaei-Rad, and Michael I. Friswell. "The mechanical behavior of composite corrugated core coated with elastomer for morphing skins". In: Journal of Composite Materials 48 (2014), pp. 1623-1636.

[5] Jack Reany and Joachim L. Grenestedt. "Corrugated skin in a foam core sandwich panel". In: Composite Structures 89 (2009), pp. 345-355.

[6] M. Golzar and P. Ghabezi. "Corrugated Composite Skins". In: Mechanics of Composite Materials 50, No. 2 (May 2014), pp. 137-148.

[7] D. E. McFarland. "An Investigation of the Static Stability of Corrugated Rectangular Plates Loaded in Pure Shear". MA thesis. University of Kansas, Lawrence, 1967.

[8] Asokendu Samanta and Madhujit Mukhopadhyay. "Finite element static and dynamic analyses of folded plates". In: Engineering Structures 21 (1999), pp. $277-287$. 
[9] Yuying Xia and Michael I. Friswell. "Equivalent Models of Corrugated Laminates for Morphing Skins". In: Active and Passive Smart Structures and Integrated Systems 7977 (2011). DOI: 10.1117/12.880433.

[10] A.T. Nettles. "Basic Mechanics of Laminated Composite Plates". In: NASA Reference Publication 1351 (1994), p. 103.

[11] Iman Dayyani, Saeed Ziaei-Rad, and Hamid Salehi. "Numerical and Experimental Investigation on Mechanical Behavior of Composite Corrugated Core". In: Applied Composite Materials 19 (2012), pp. 705-721. DOI: 10 . 1007/s10443-011-9238-3.

[12] Michael Christian Winkler. "Analysis of Corrugated Laminates". MA thesis. ETH Zurich, 2012.

[13] Renee Lee. Woven Fabric: Types of Weaves. URL: https://www.linkedin. com/pulse/woven-fabric-types-weaves-renee-lee. 


\section{APPENDIX A FEA MATERIAL PROPERTIES}

Table A.1: Material Properties for FEA Models

\begin{tabular}{|c|c|c|c|}
\hline Property & FG (MXB 7781) & CF (M46J) & Stainless Steel \\
\hline$E_{1}[\mathrm{psi}]$ & 3514060 & $3.4 \mathrm{E} 7$ & $2.8 \mathrm{E} 7$ \\
$E_{2}[\mathrm{psi}]$ & 3514060 & 910000 & - \\
$E_{3}[\mathrm{psi}]$ & $1.395 \mathrm{E} 6$ & 910000 & - \\
$\nu_{12}$ & 0.11 & 0.3 & 0.29 \\
$\nu_{13}$ & 0.318 & 0.3 & - \\
$\nu_{23}$ & 0.318 & 0.217 & - \\
$G_{12}[\mathrm{psi}]$ & 747700 & 117000 & - \\
$G_{13}[\mathrm{psi}]$ & 405400 & 117000 & - \\
$G_{23}[\mathrm{psi}]$ & 405400 & 374000 & - \\
\hline
\end{tabular}




\section{APPENDIX B LOAD-DEFORMATION CURVES}

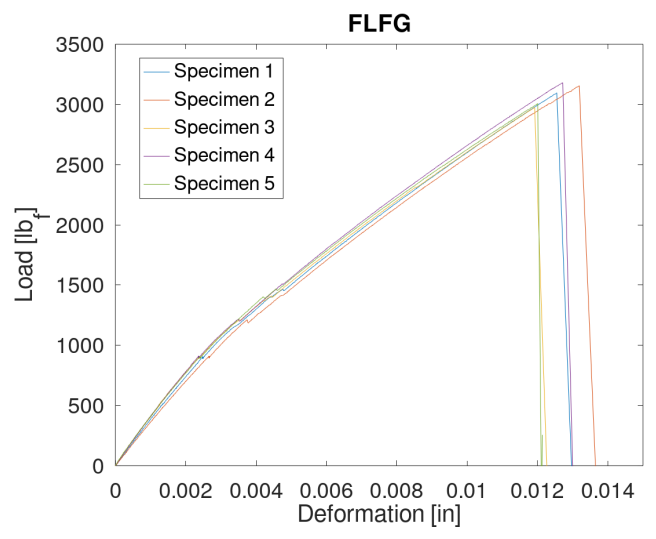

(a) Flat Longitudinal FG

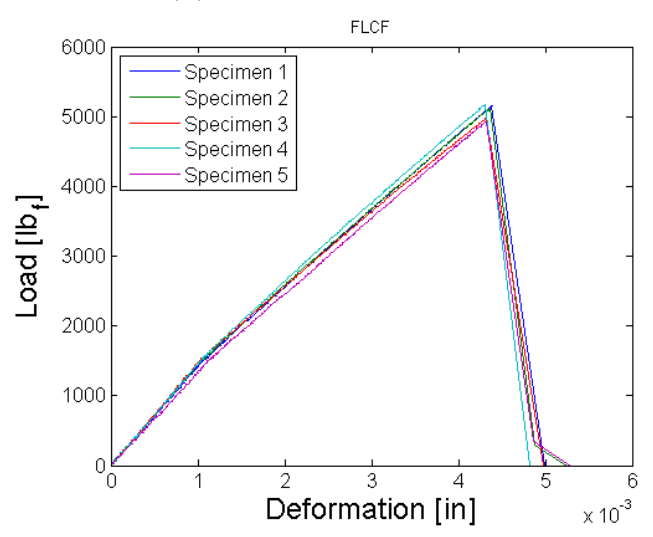

(c) Flat Longitudinal $\mathrm{CF}$

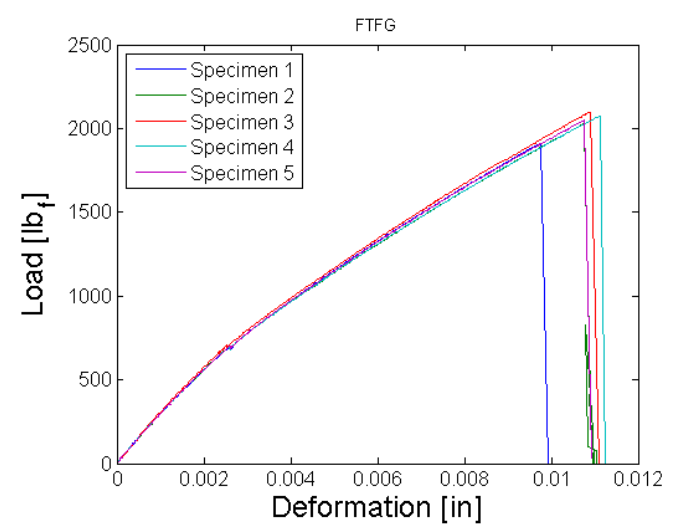

(b) Flat Transverse FG

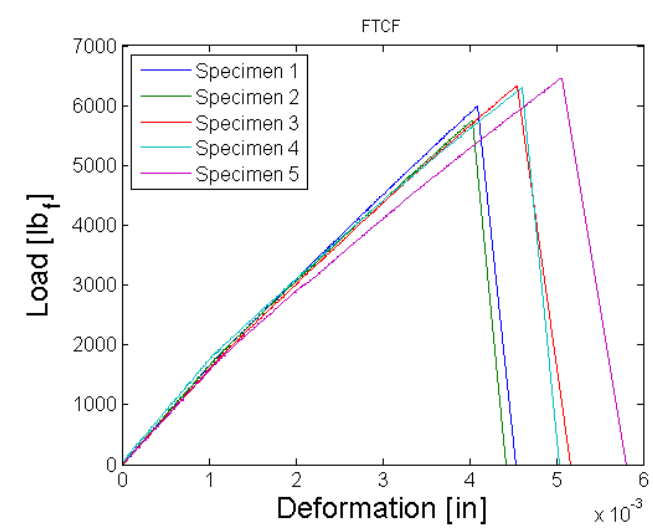

(d) Flat Transverse CF

Figure B.1: Load-Deformation Curves of Flat Tensile Specimens 


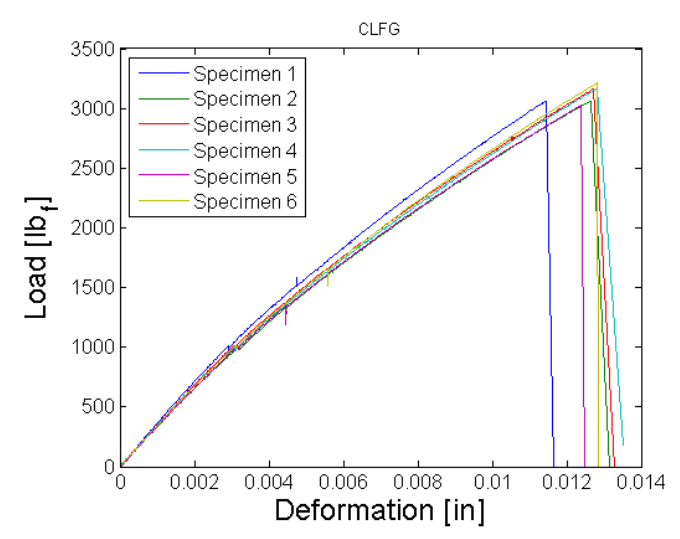

(a) Corrugated Longitudinal FG

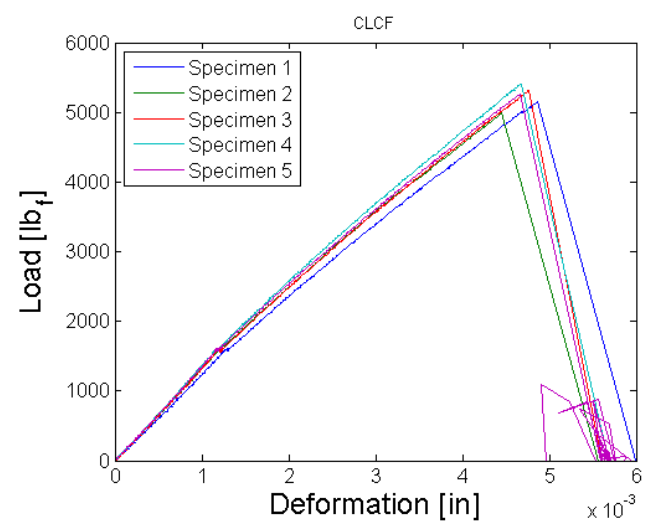

(c) Corrugated Longitudinal CF

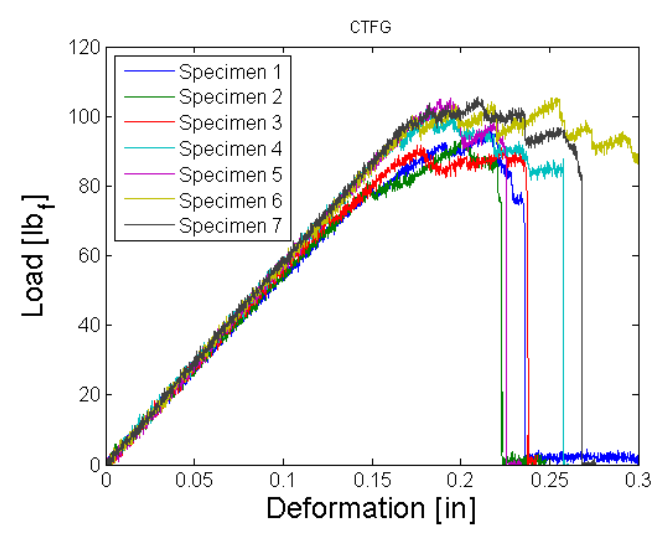

(b) Corrugated Transverse FG

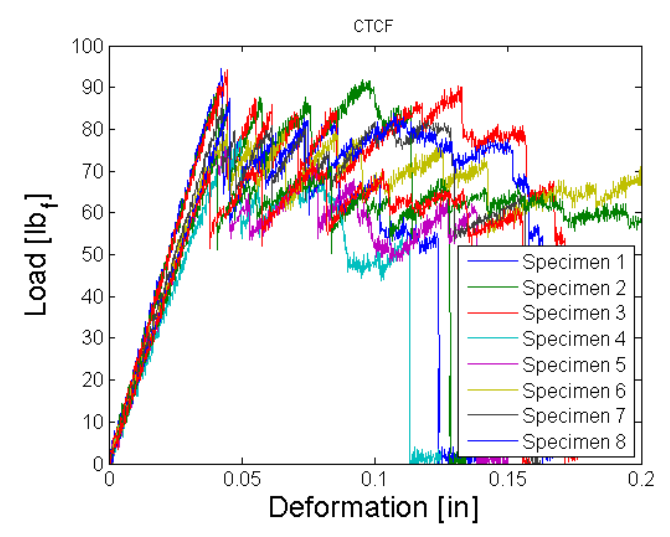

(d) Corrugated Transverse CF

Figure B.2: Load-Deformation Curves of Corrugated Tensile Specimens 


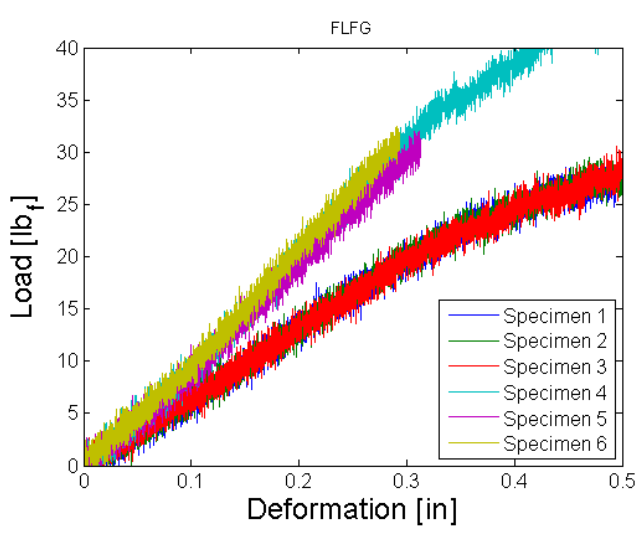

(a) Flat Longitudinal FG

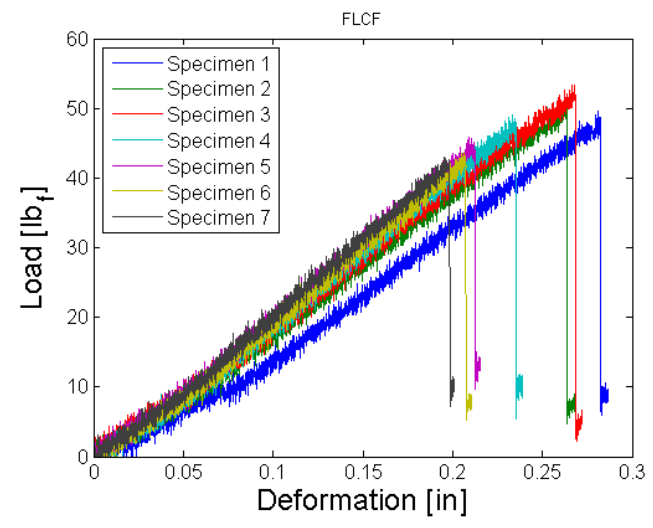

(c) Flat Longitudinal $\mathrm{CF}$

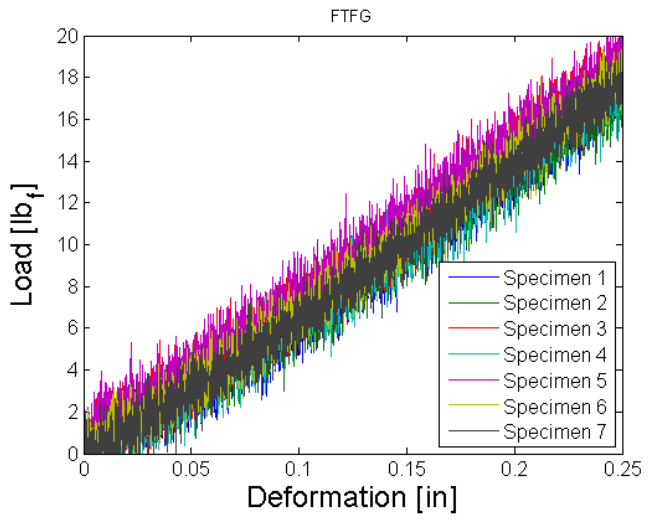

(b) Flat Transverse FG

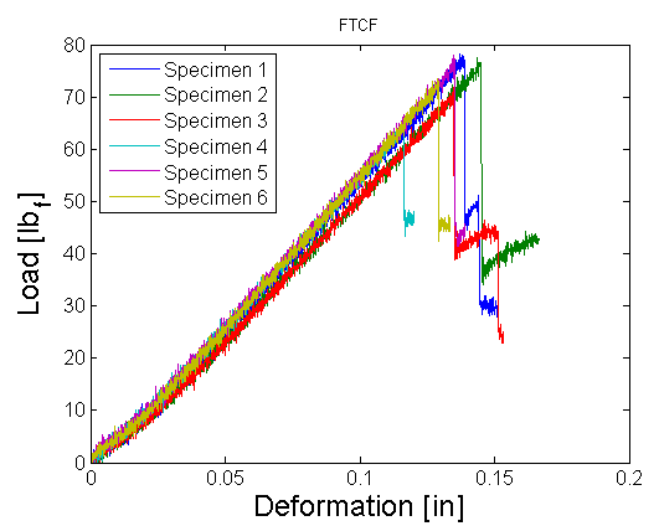

(d) Flat Transverse CF

Figure B.3: Load-Deformation Curves of Flat Flexure Specimens 


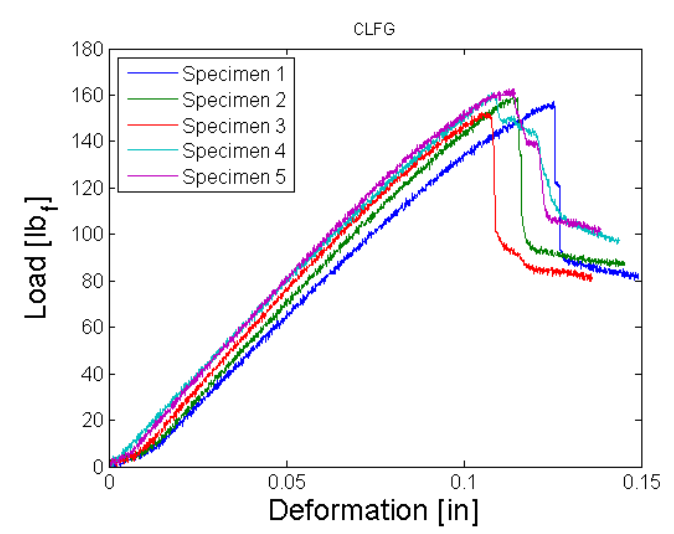

(a) Corrugated Longitudinal FG

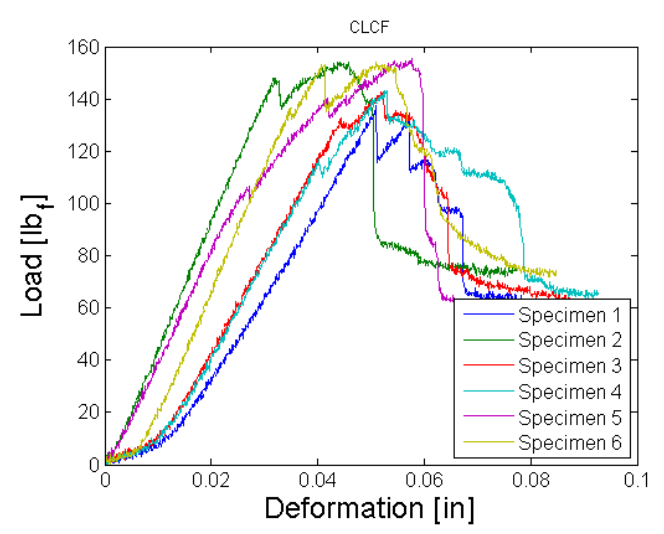

(c) Corrugated Longitudinal CF

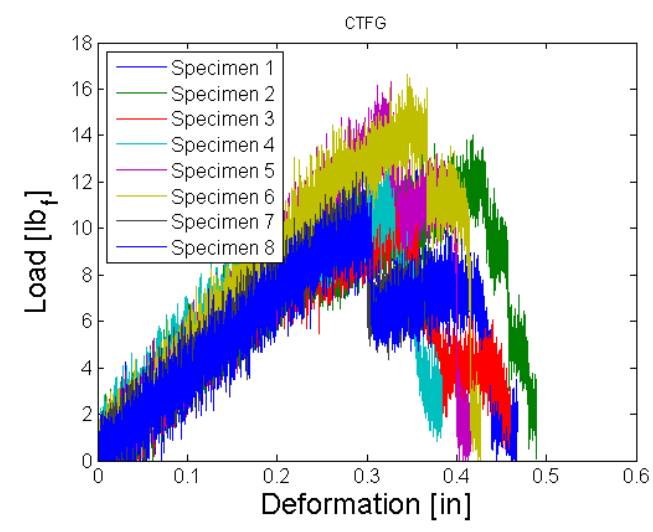

(b) Corrugated Transverse FG

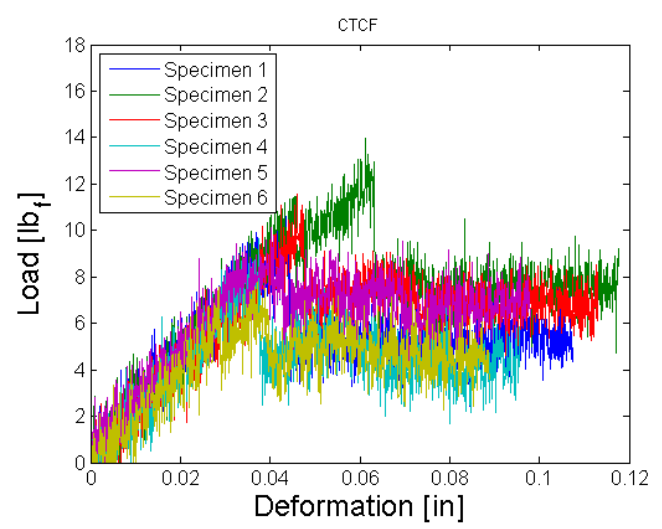

(d) Corrugated Transverse CF

Figure B.4: Load-Deformation Curves of Corrugated Flexure Specimens 


\section{APPENDIX C MESH CONVERGENCE}

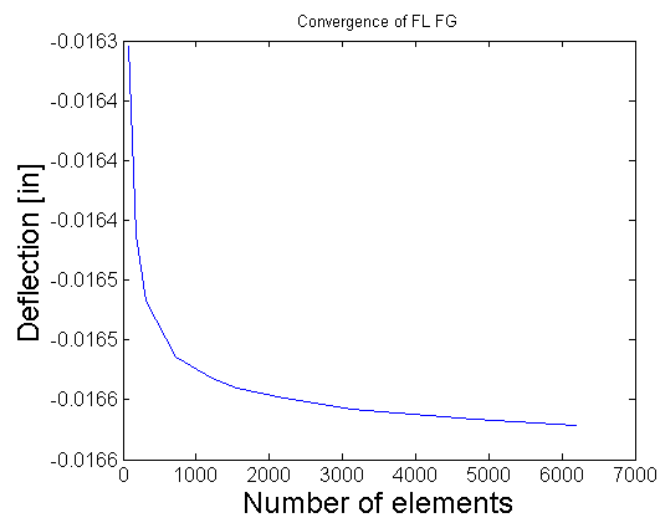

(a) Flat Longitudinal FG

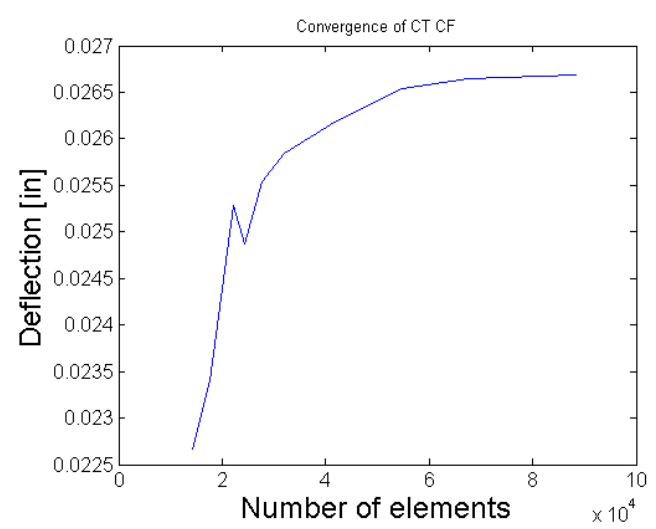

(c) Flat Longitudinal CF

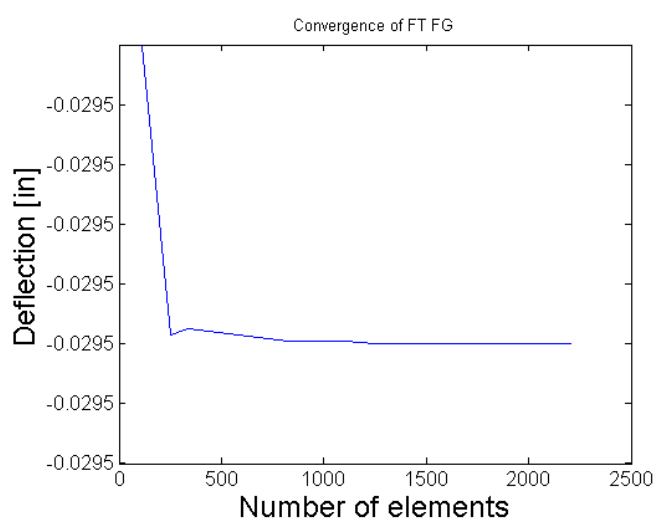

(b) Flat Transverse FG

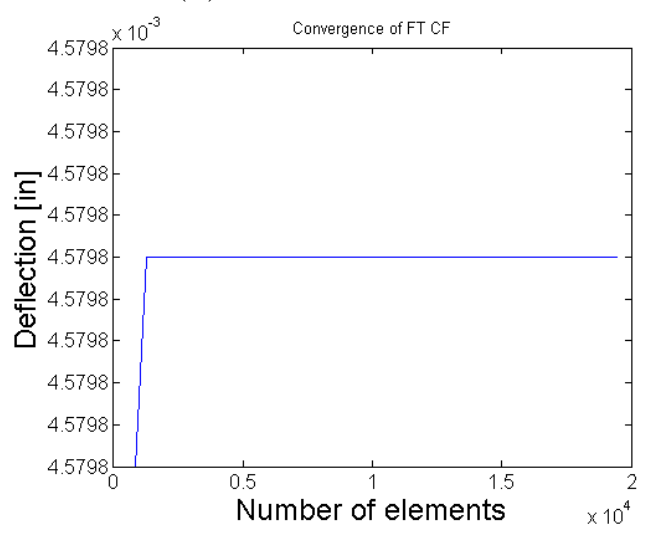

(d) Flat Transverse CF

Figure C.1: Mesh Convergence Studies for Flat Tensile Models 


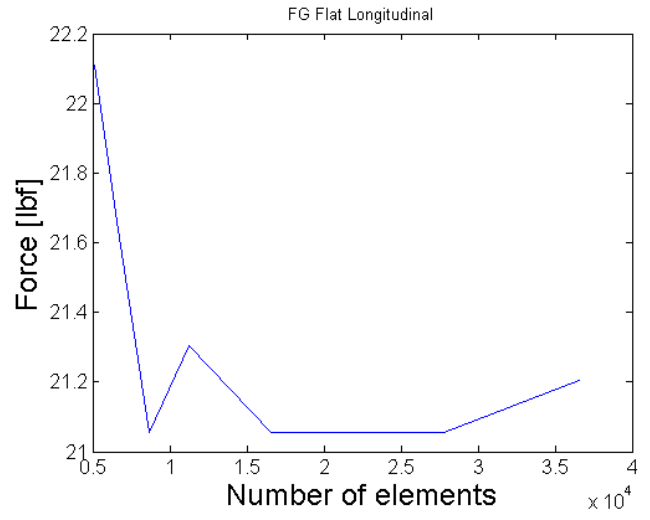

(a) Flat Longitudinal FG

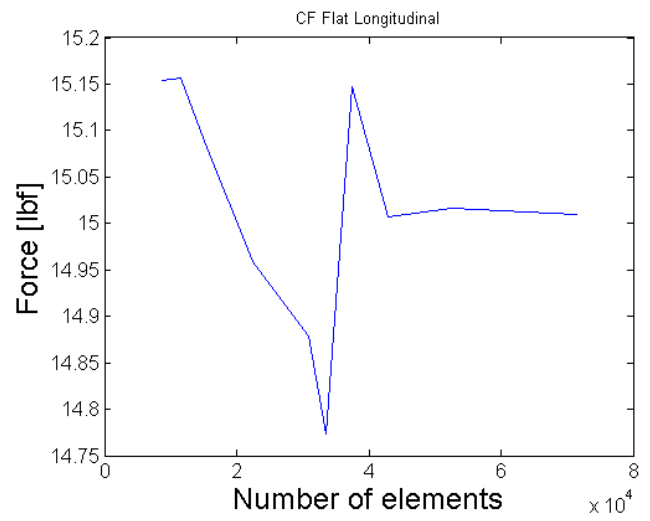

(c) Flat Longitudinal CF

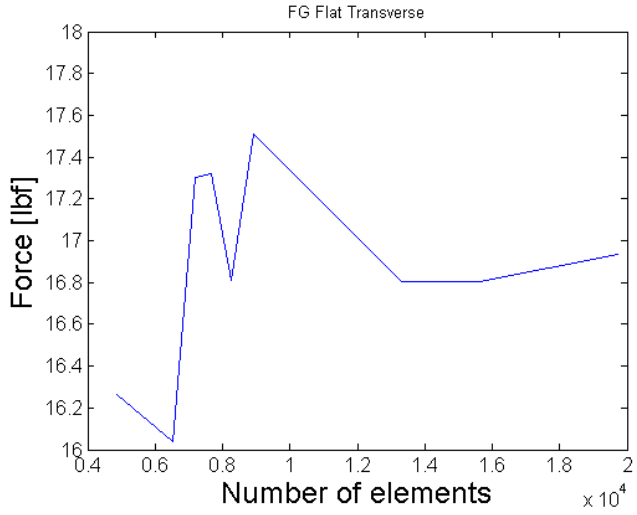

(b) Flat Transverse FG

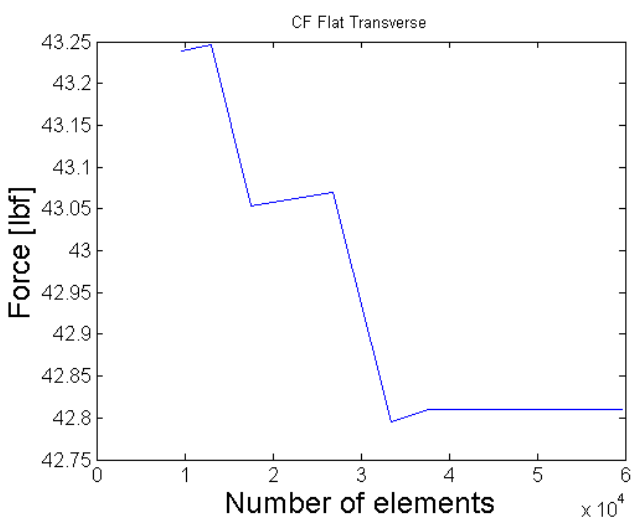

(d) Flat Transverse CF

Figure C.2: Mesh Convergence Studies for Flat Flexure Models 


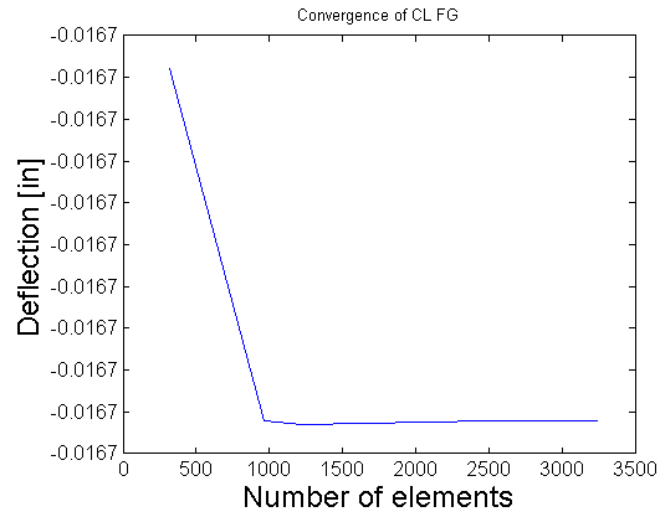

(a) Corrugated Longitudinal FG

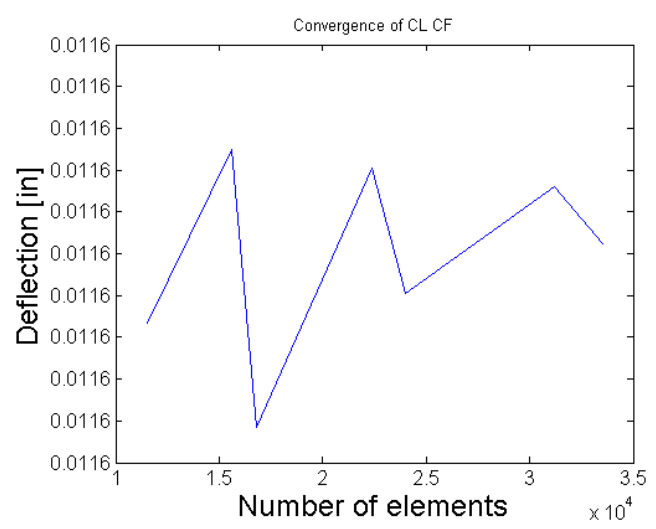

(c) Corrugated Longitudinal CF

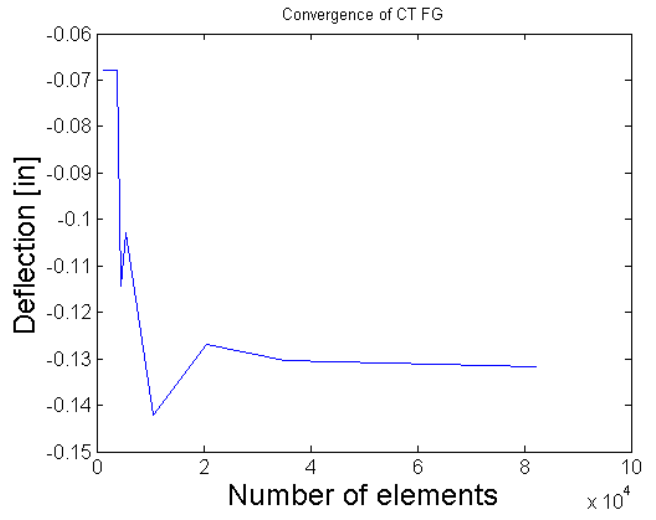

(b) Corrugated Transverse FG

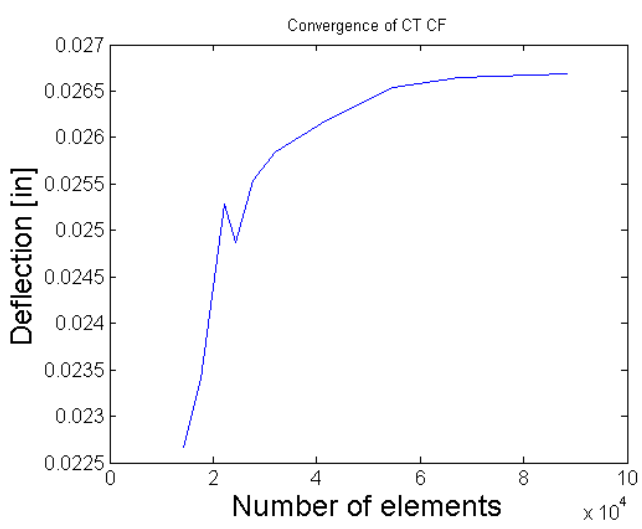

(d) Corrugated Transverse CF

Figure C.3: Mesh Convergence Studies for Corrugated Tensile Models 


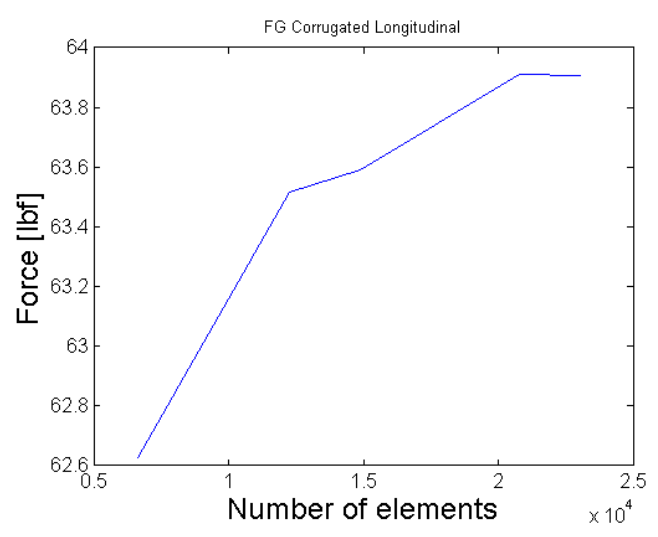

(a) Corrugated Longitudinal FG

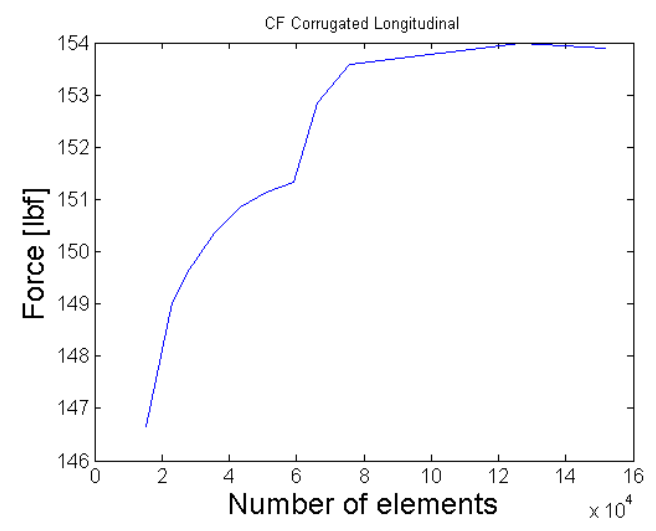

(c) Corrugated Longitudinal CF

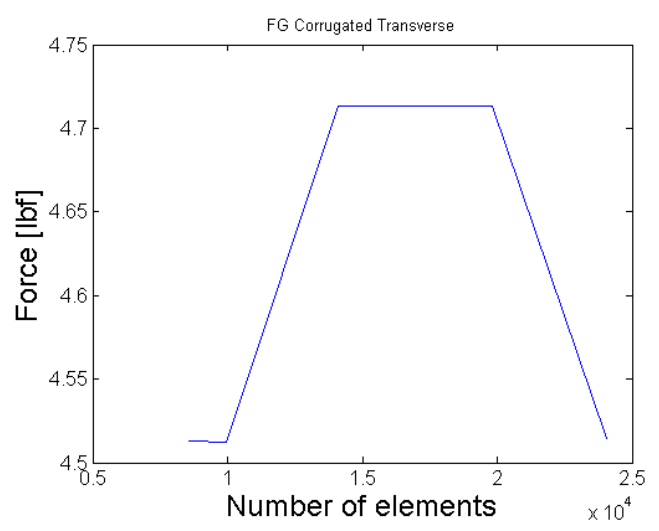

(b) Corrugated Transverse FG

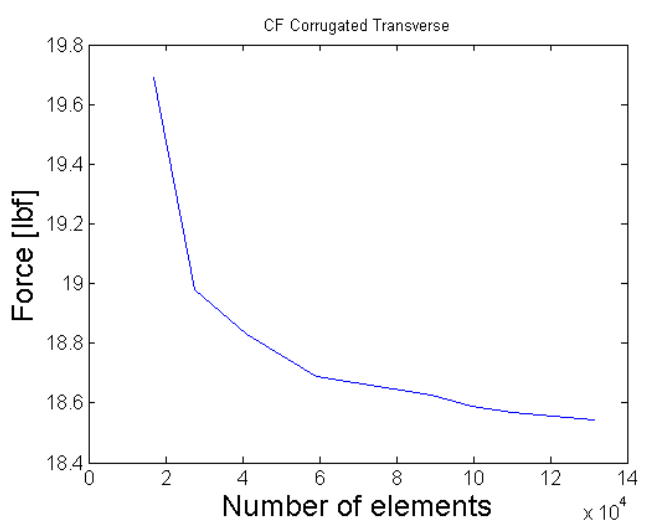

(d) Corrugated Transverse CF

Figure C.4: Mesh Convergence Studies for Corrugated Flexure Models 


\section{APPENDIX D MATLAB CODE}

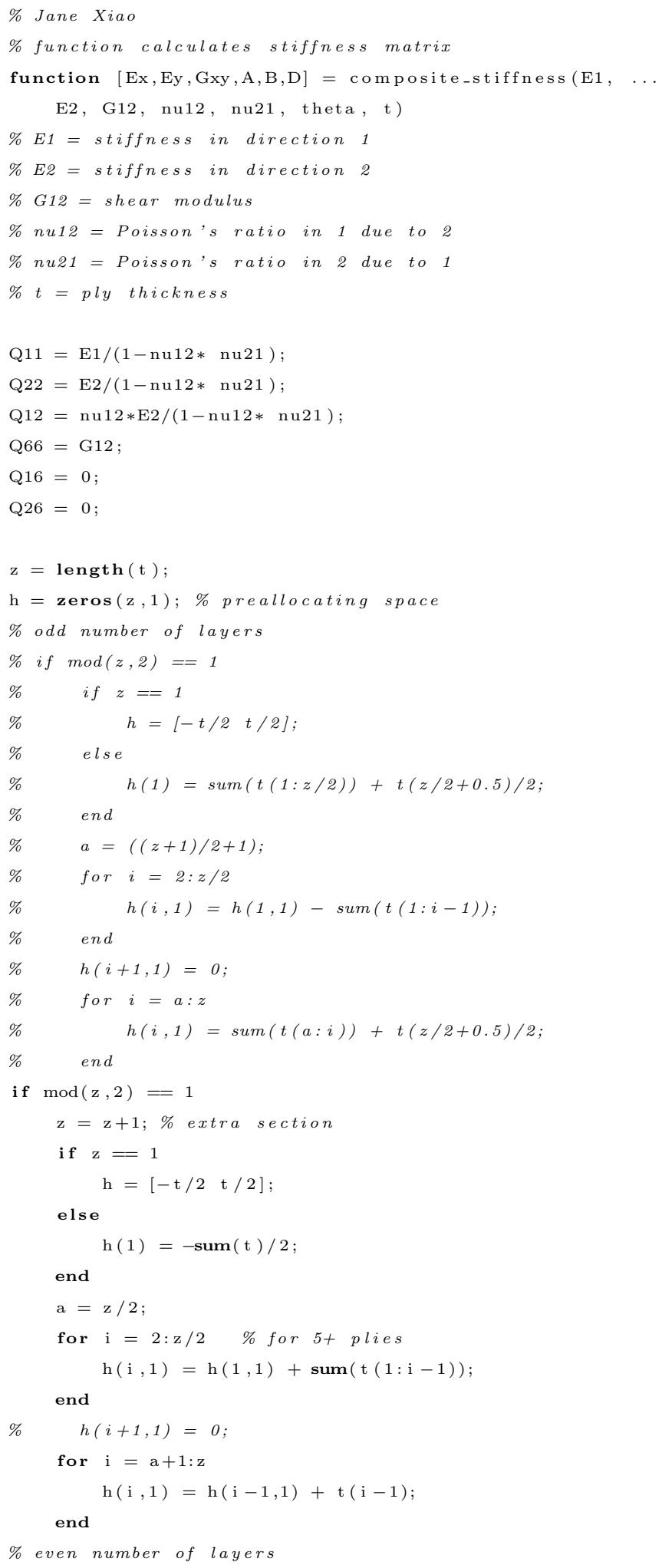




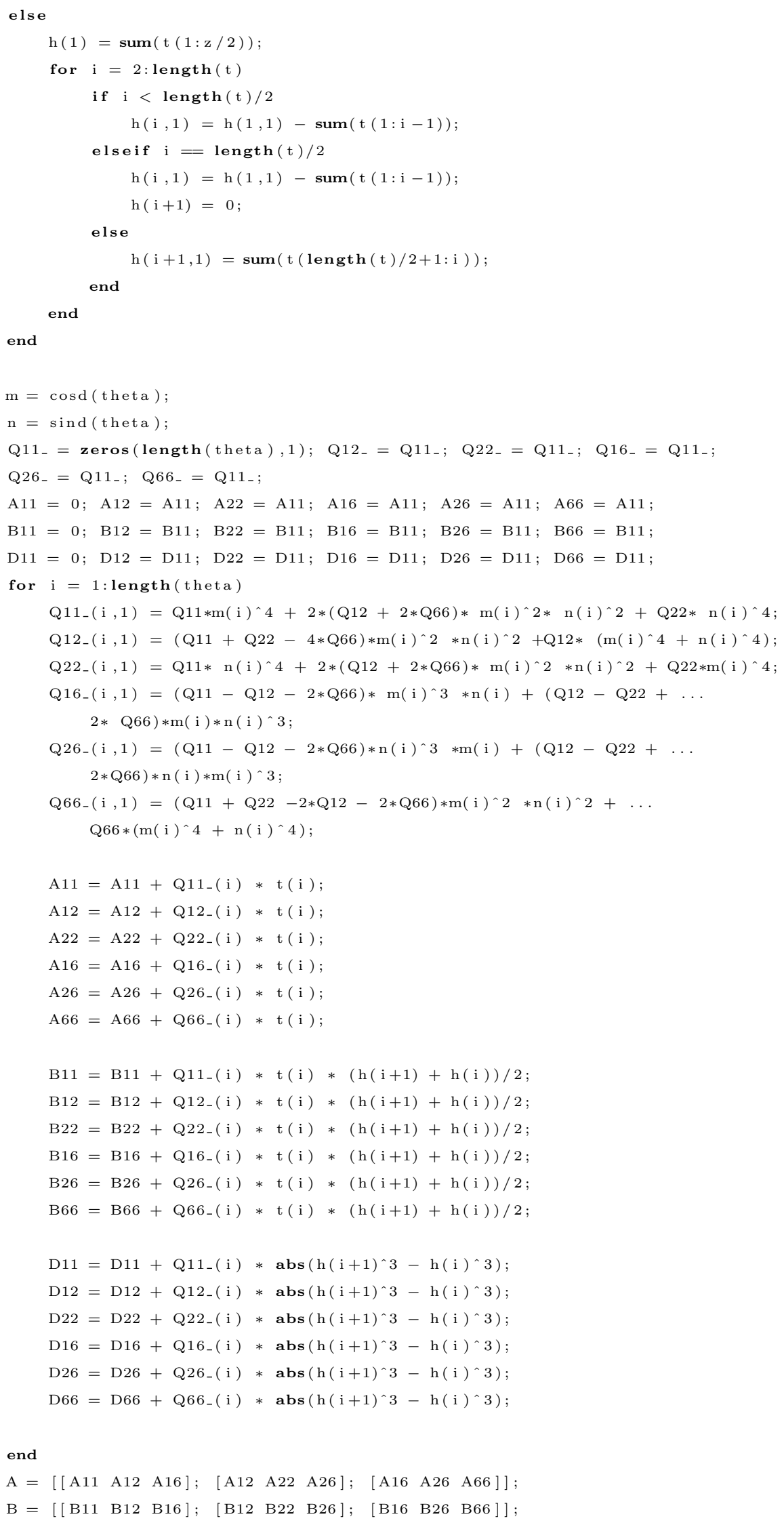




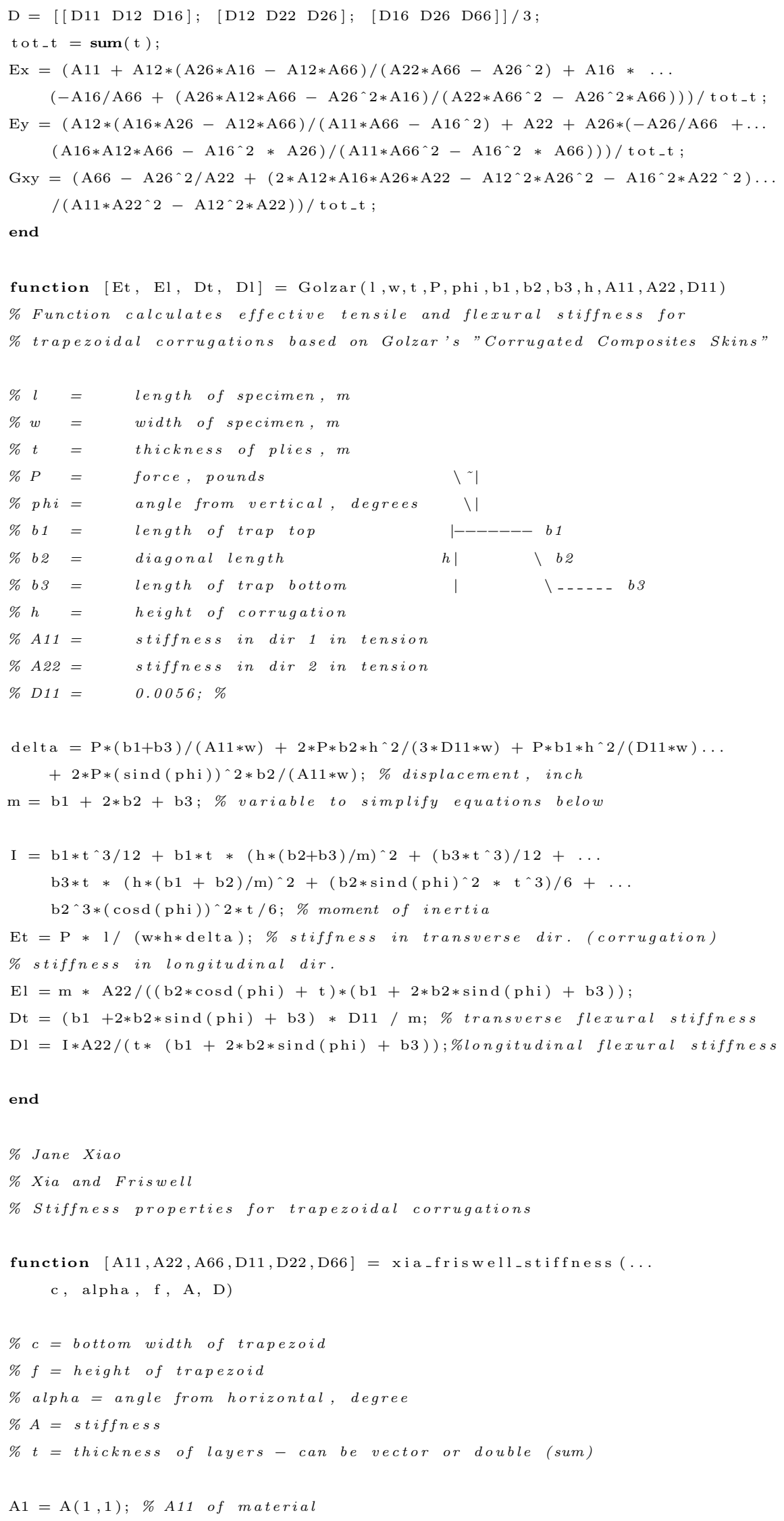




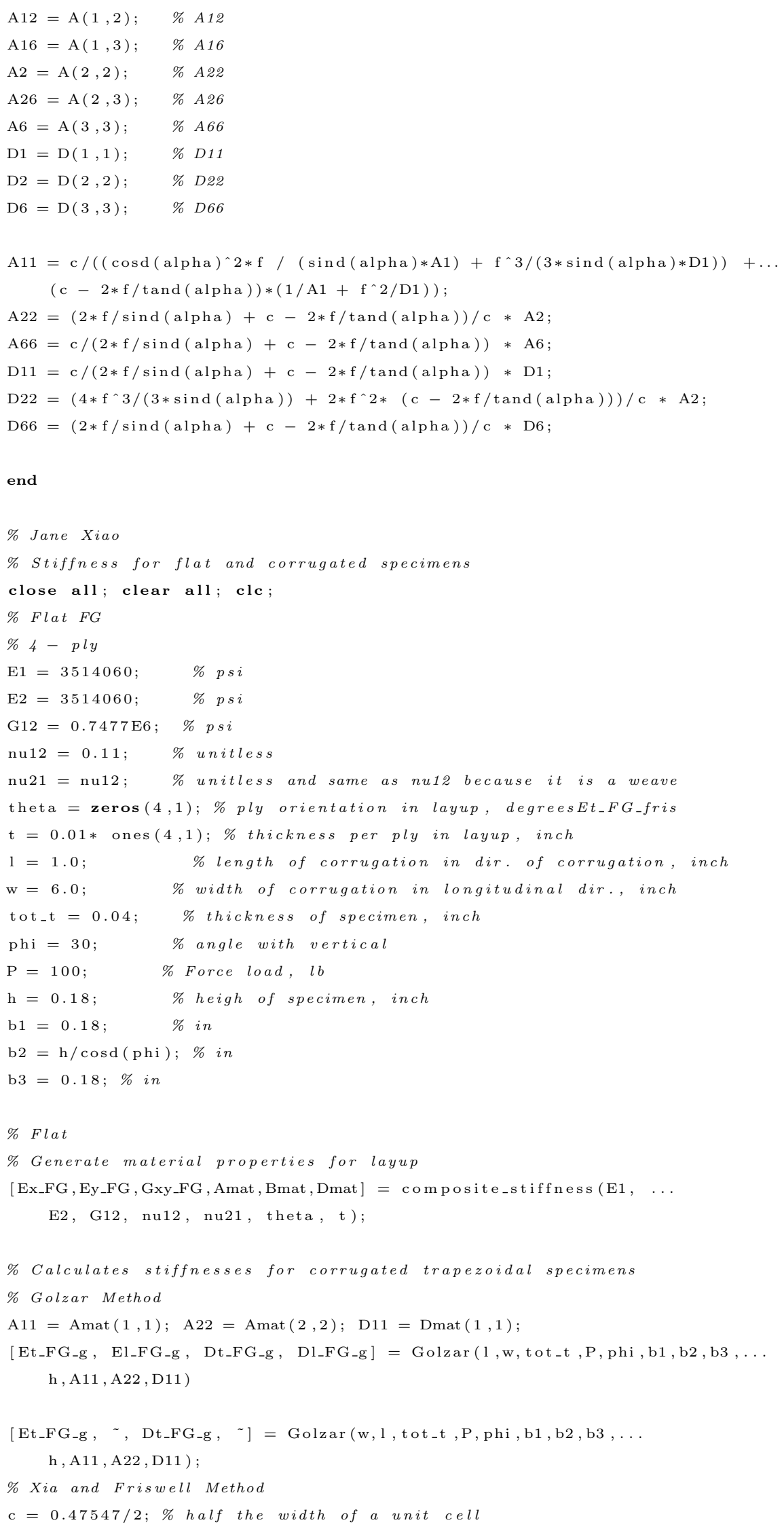




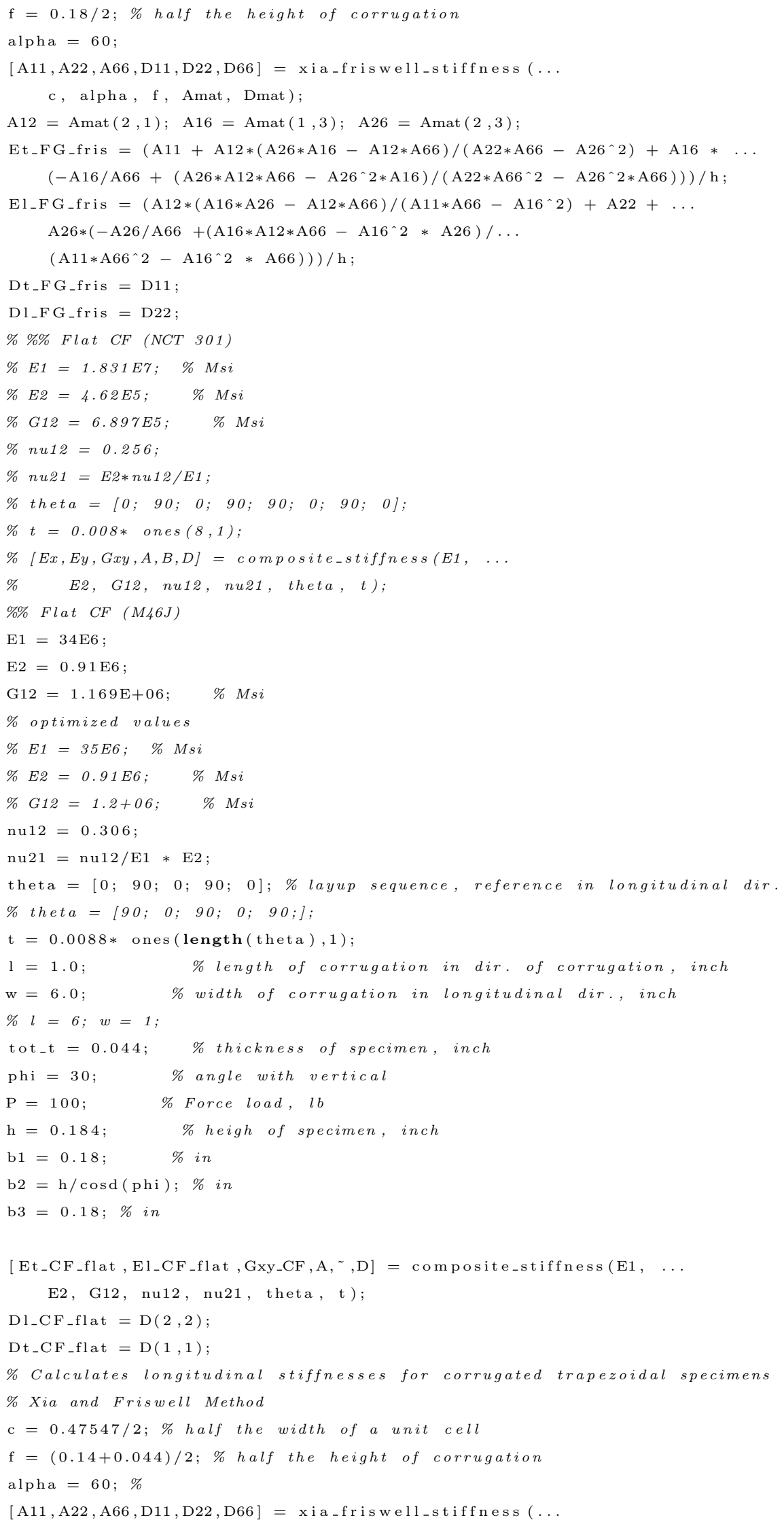




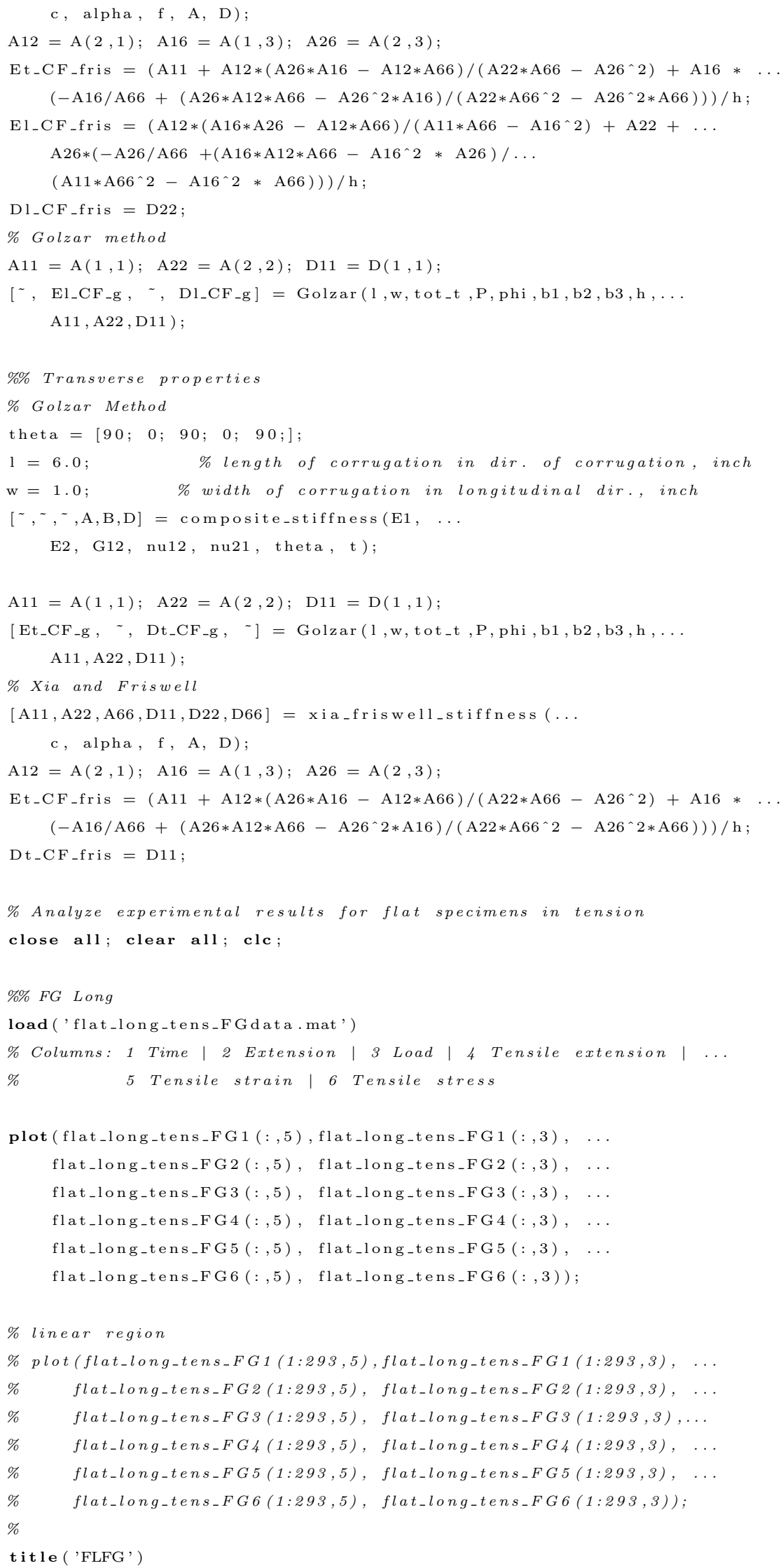




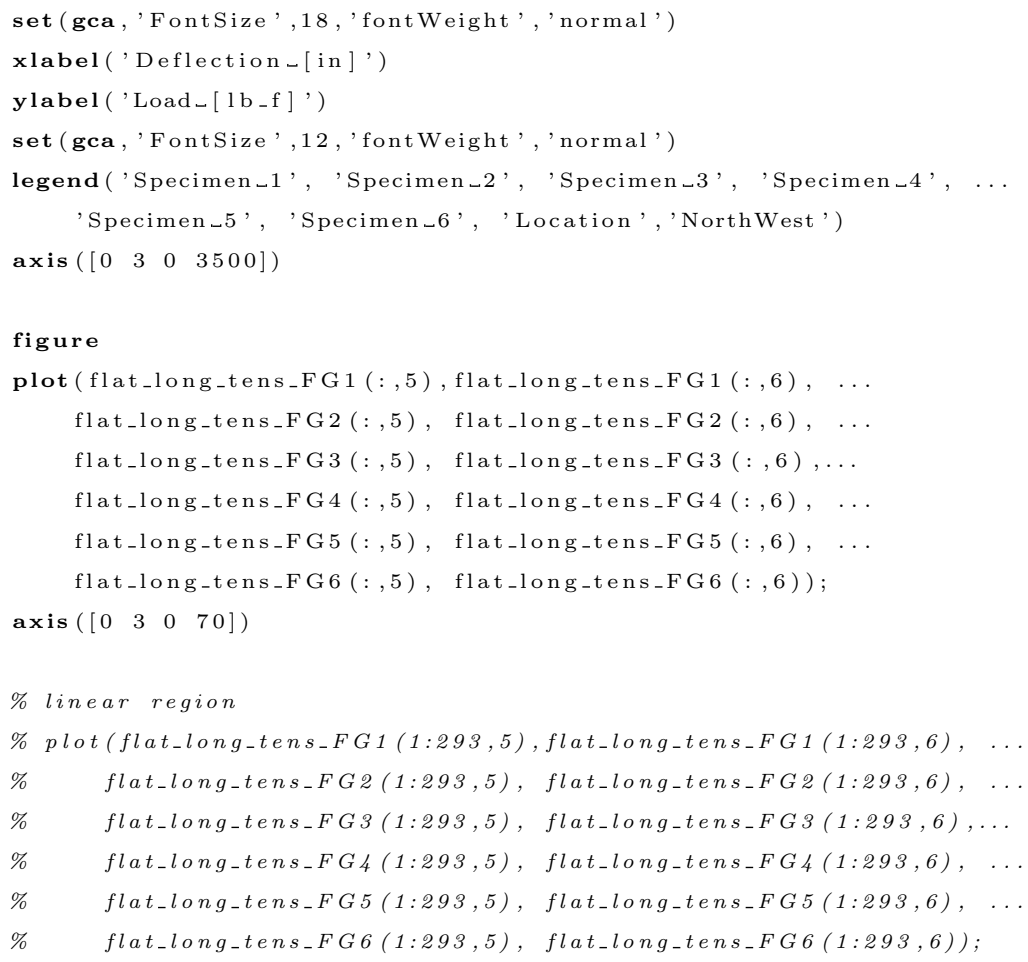




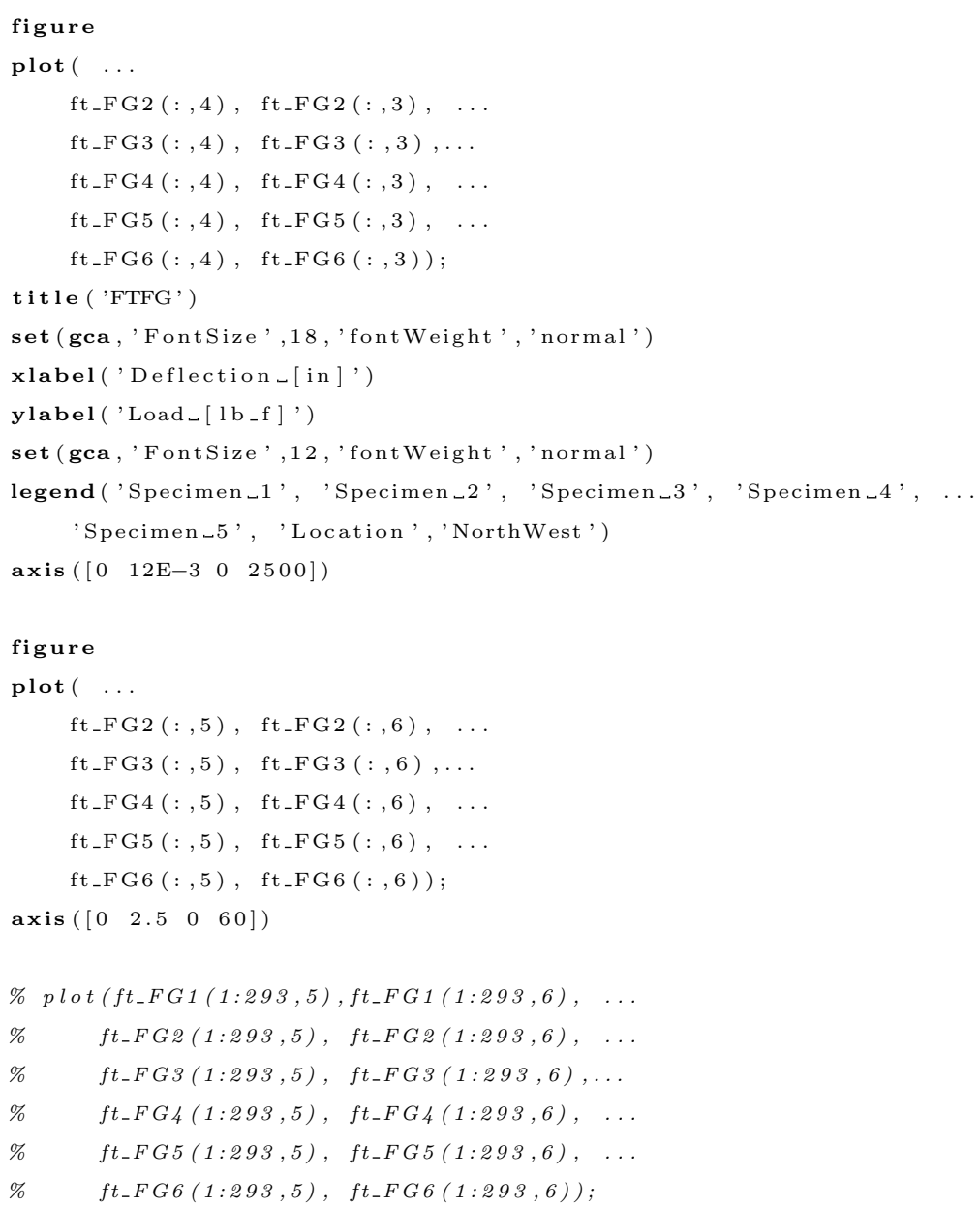




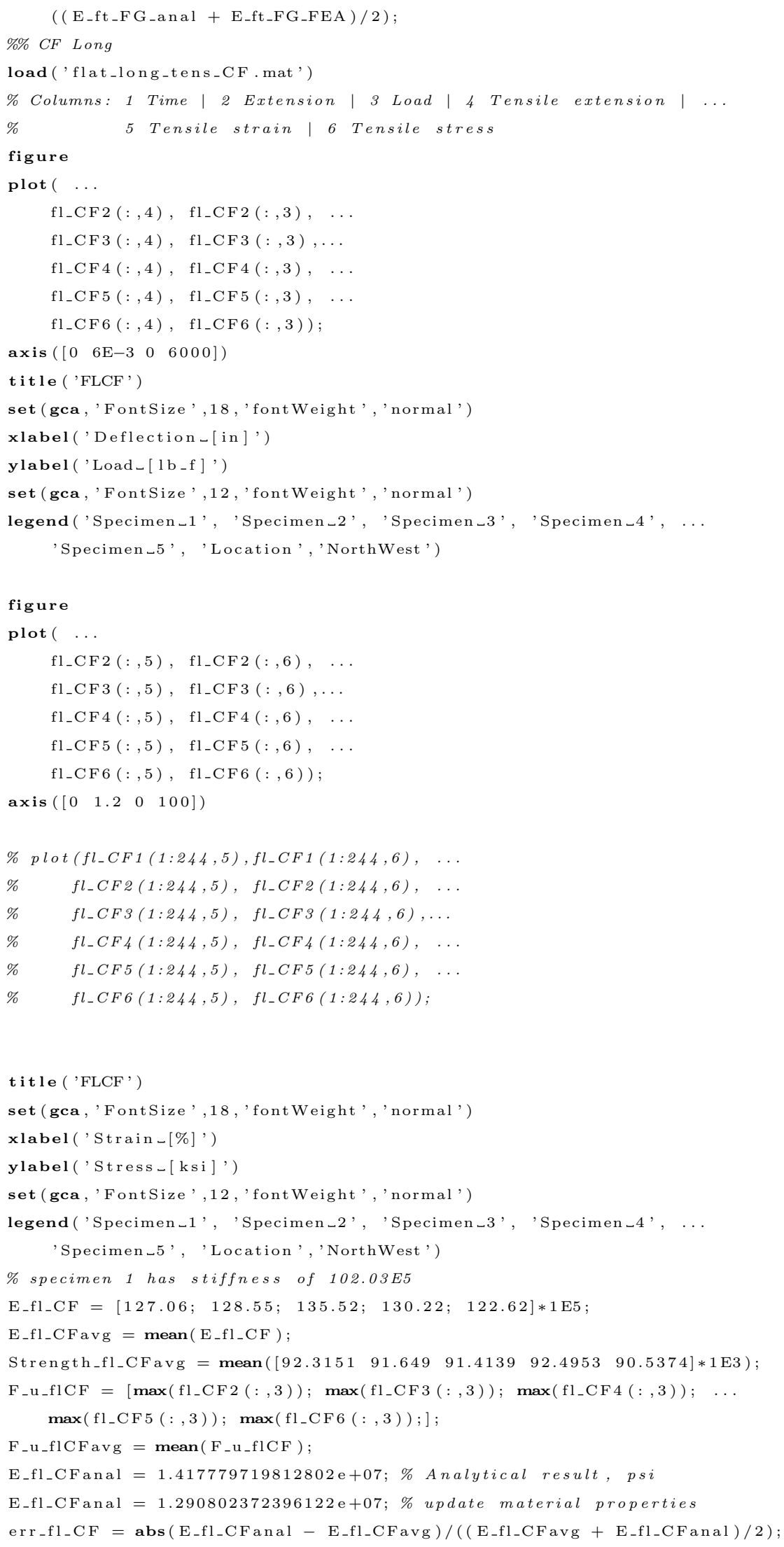




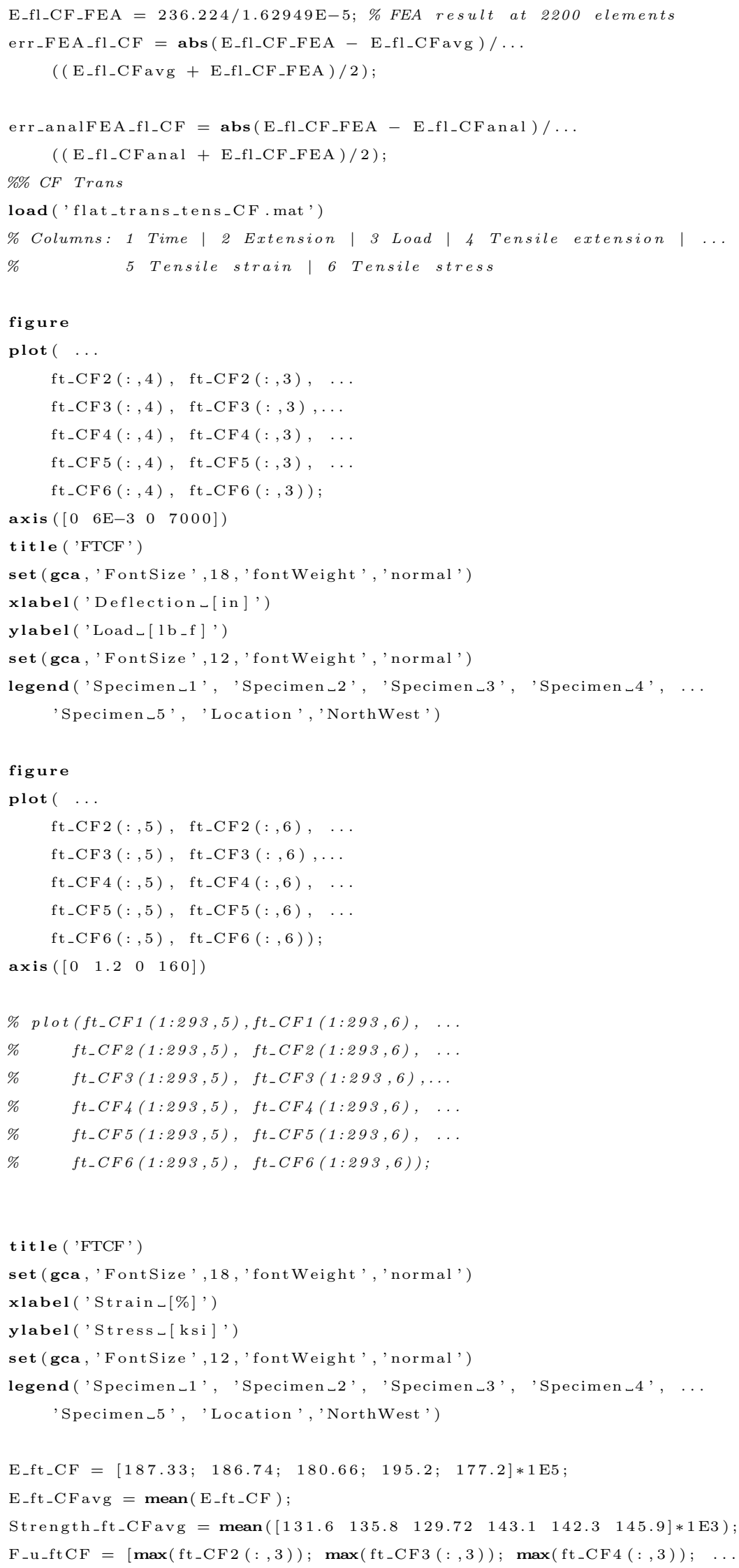




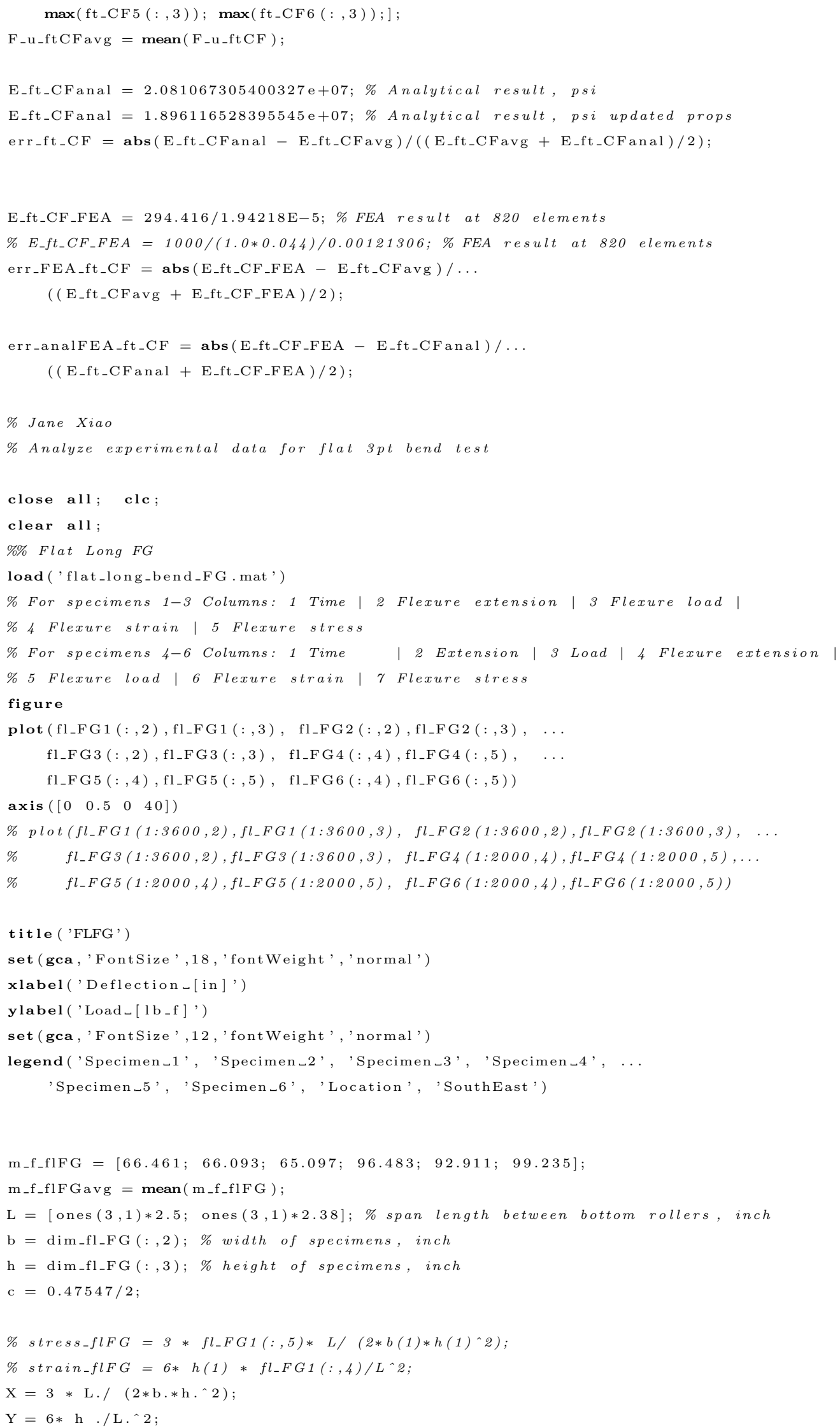




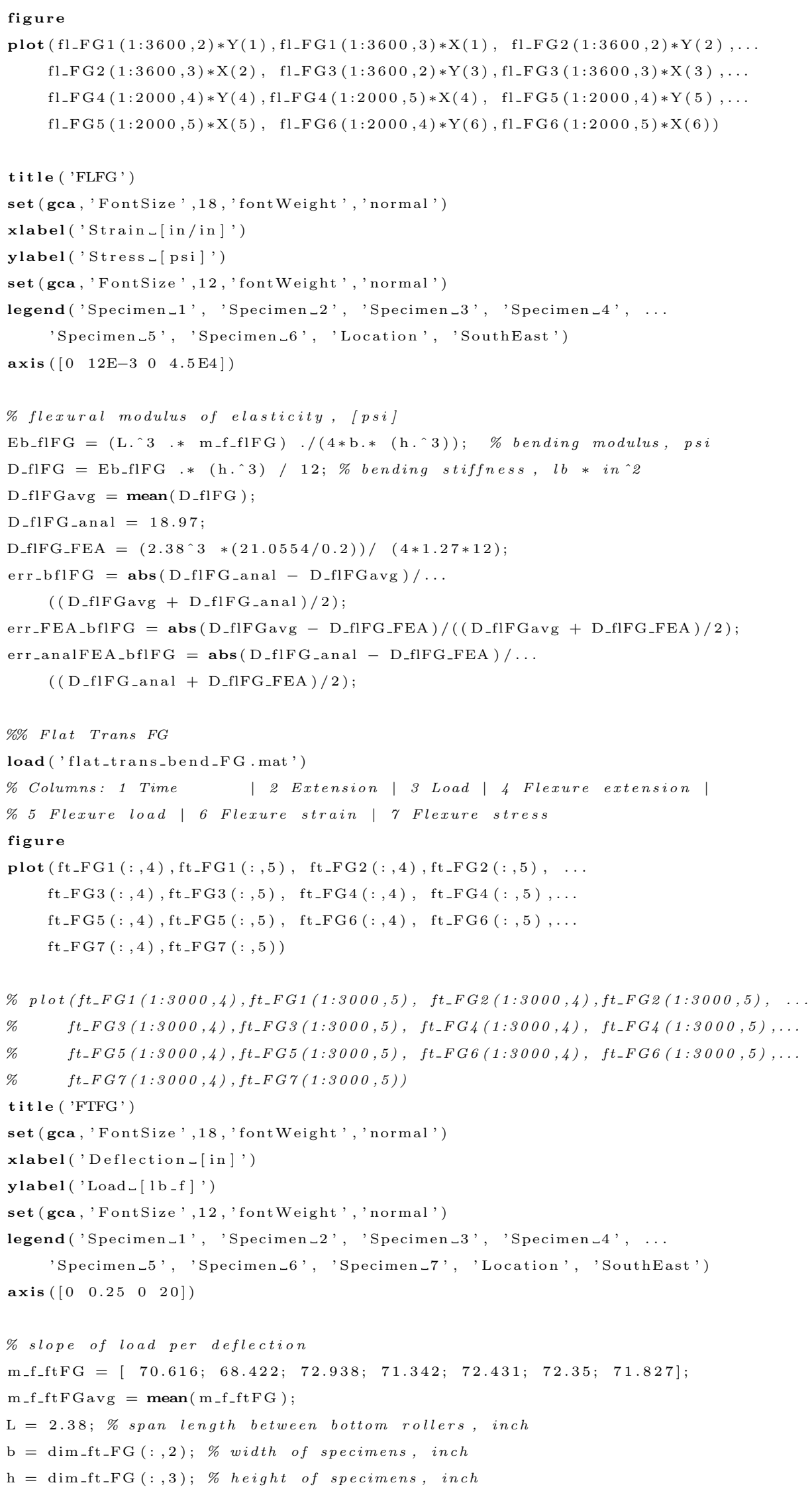




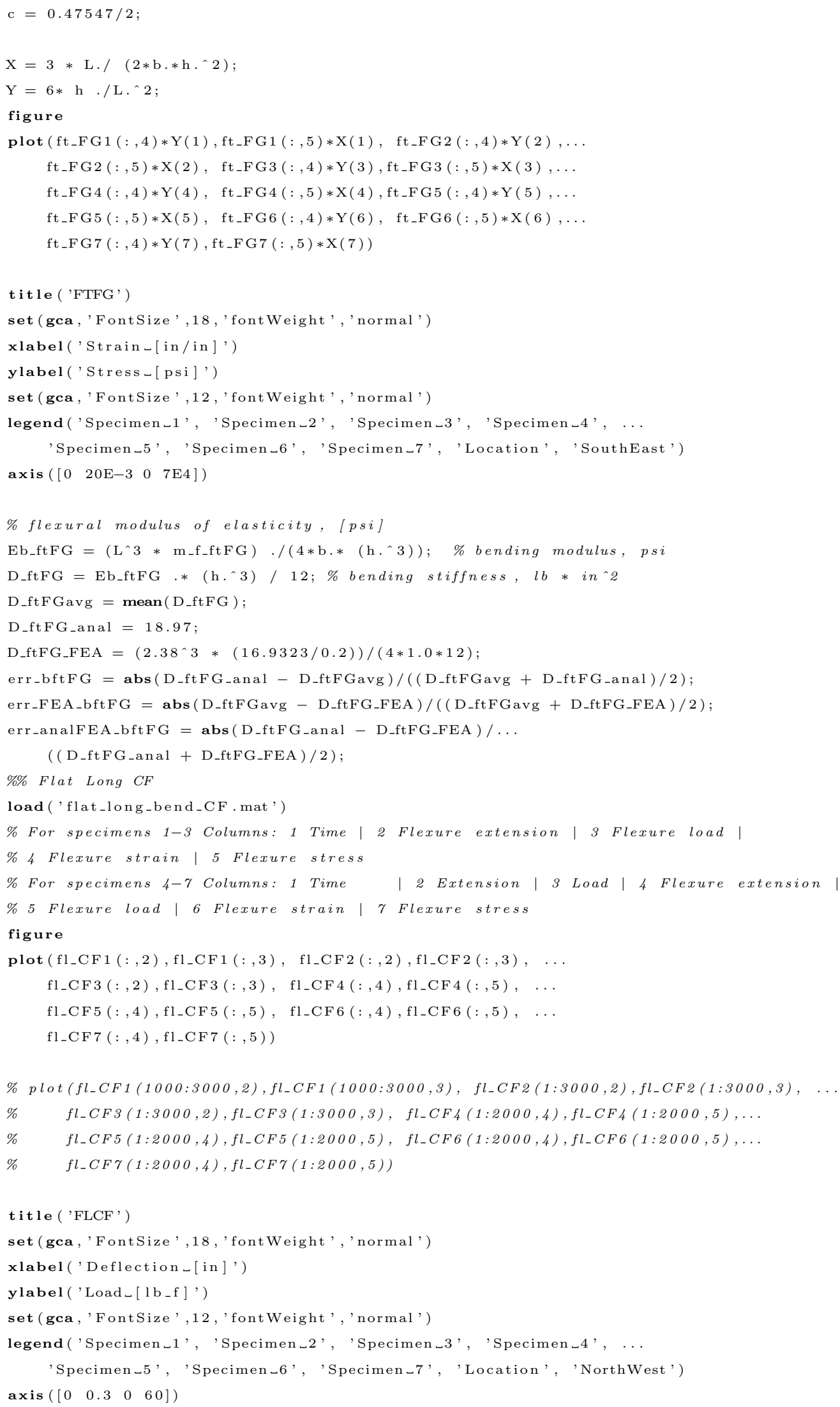




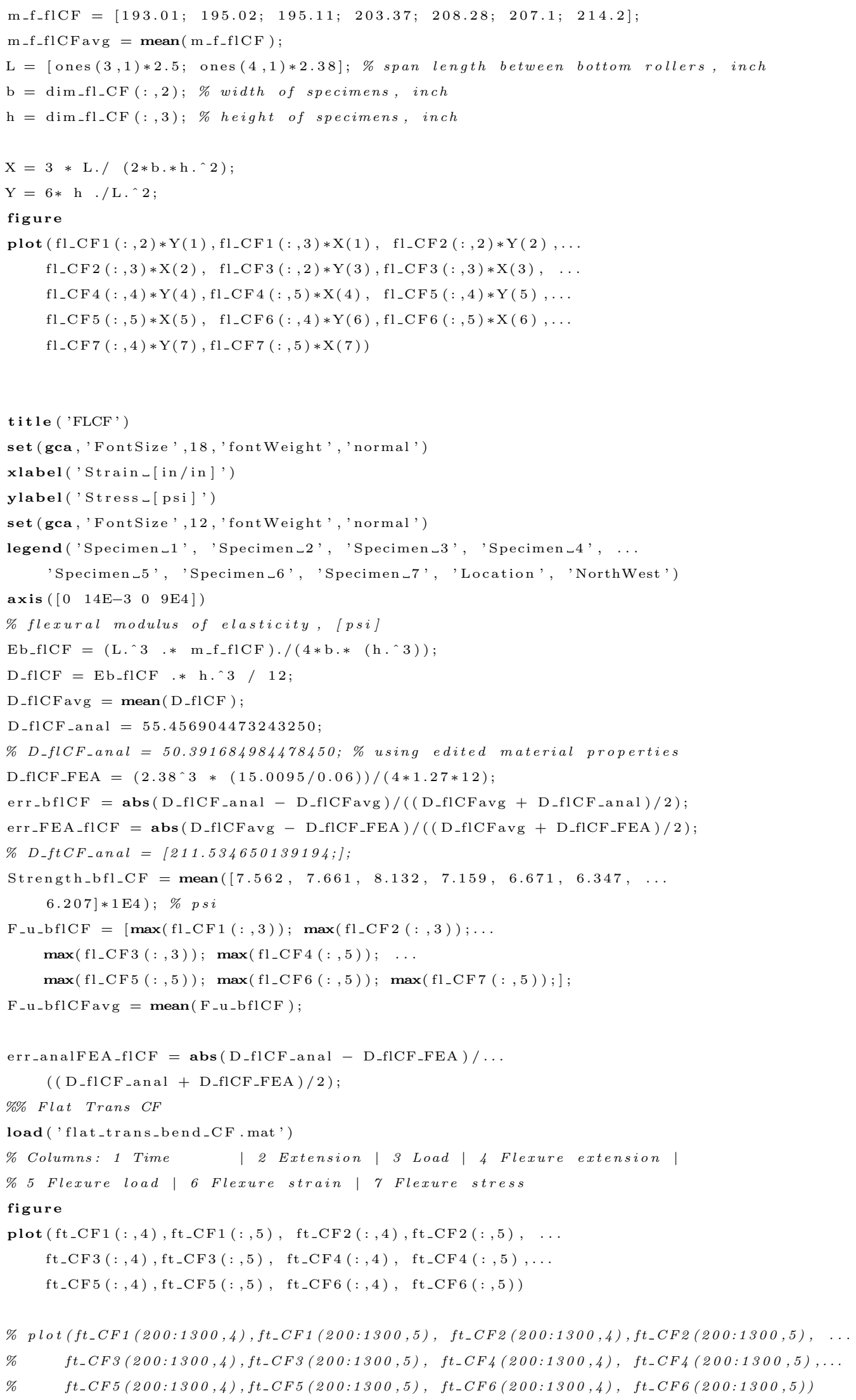




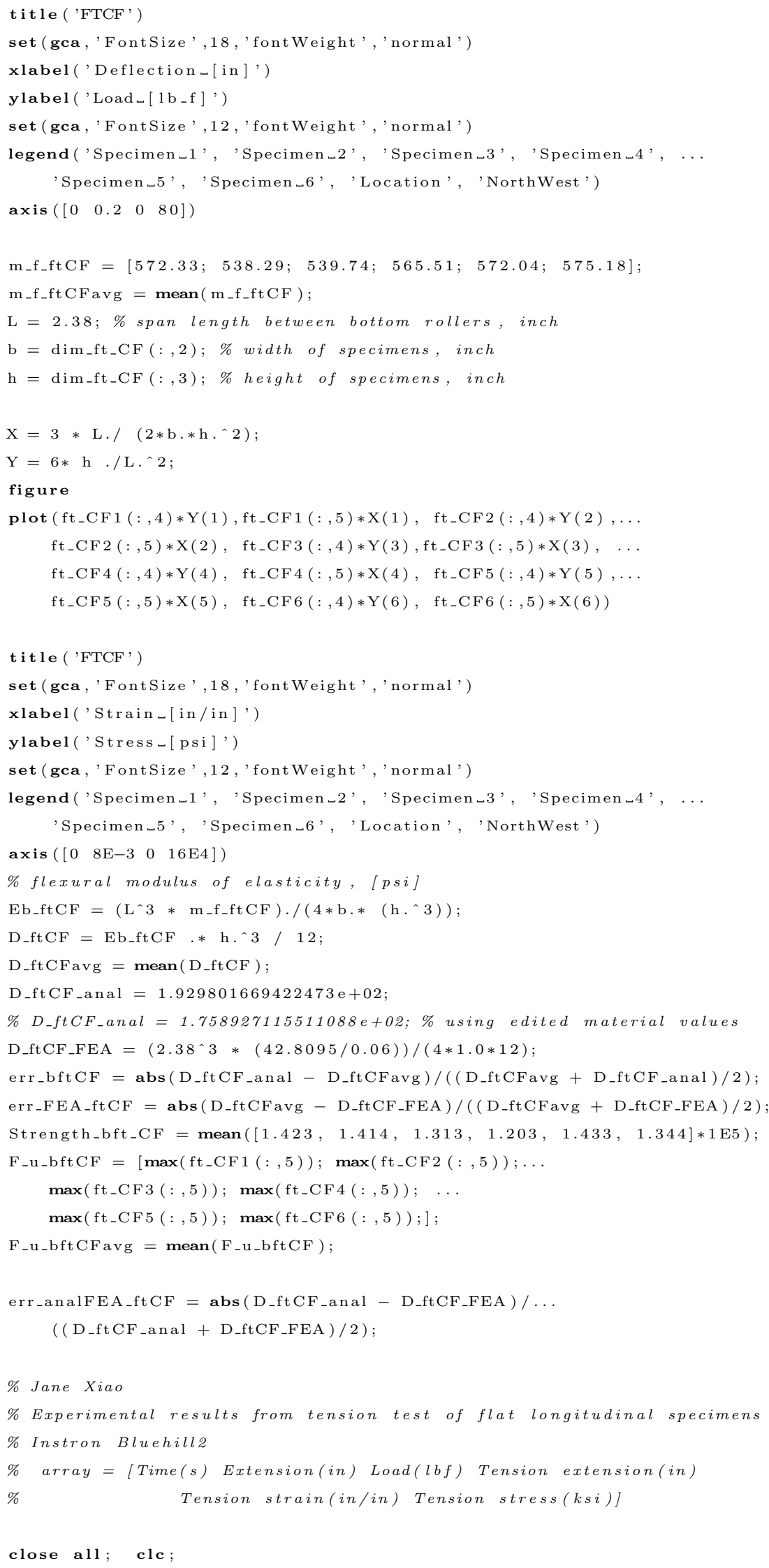




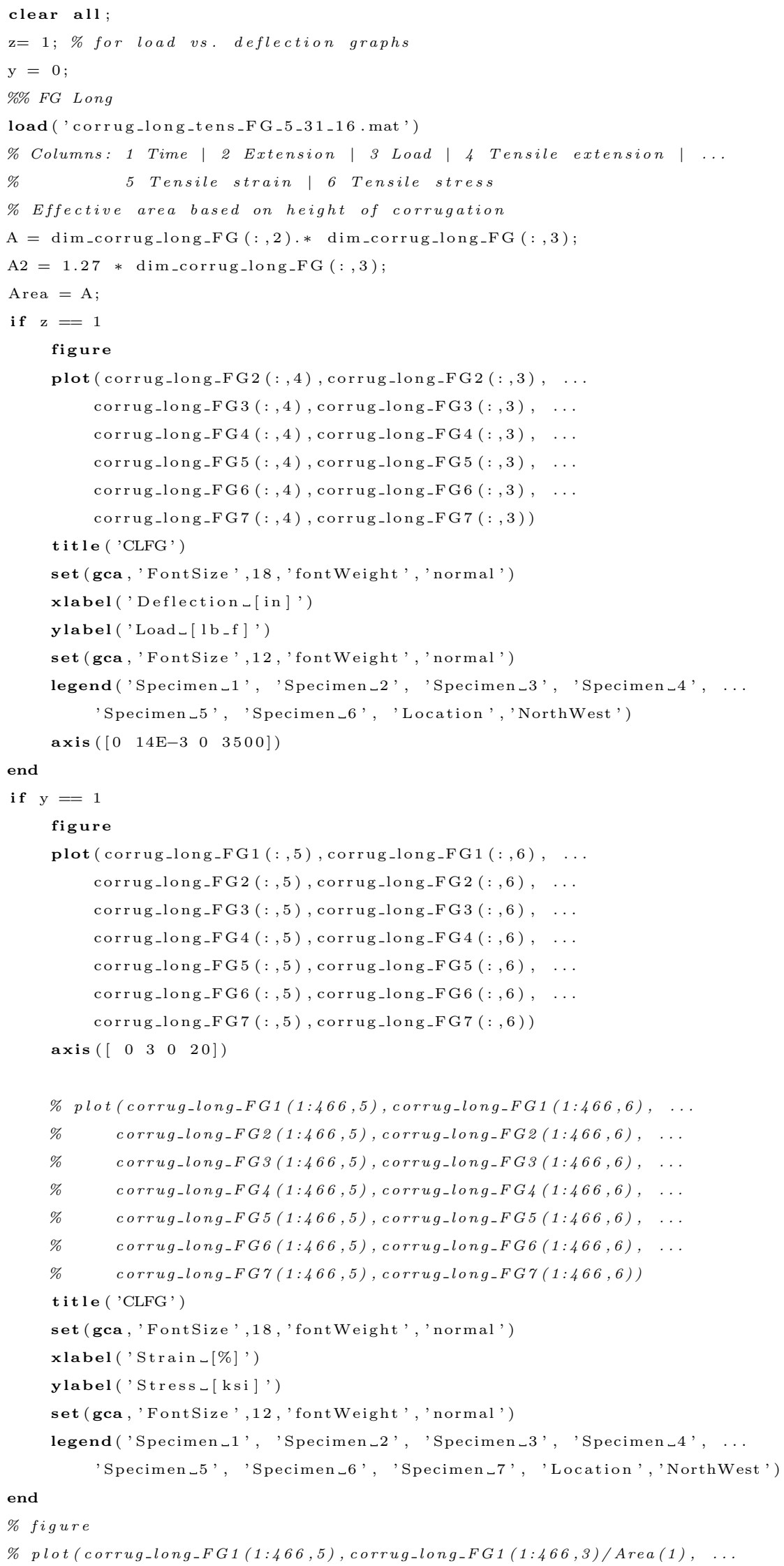




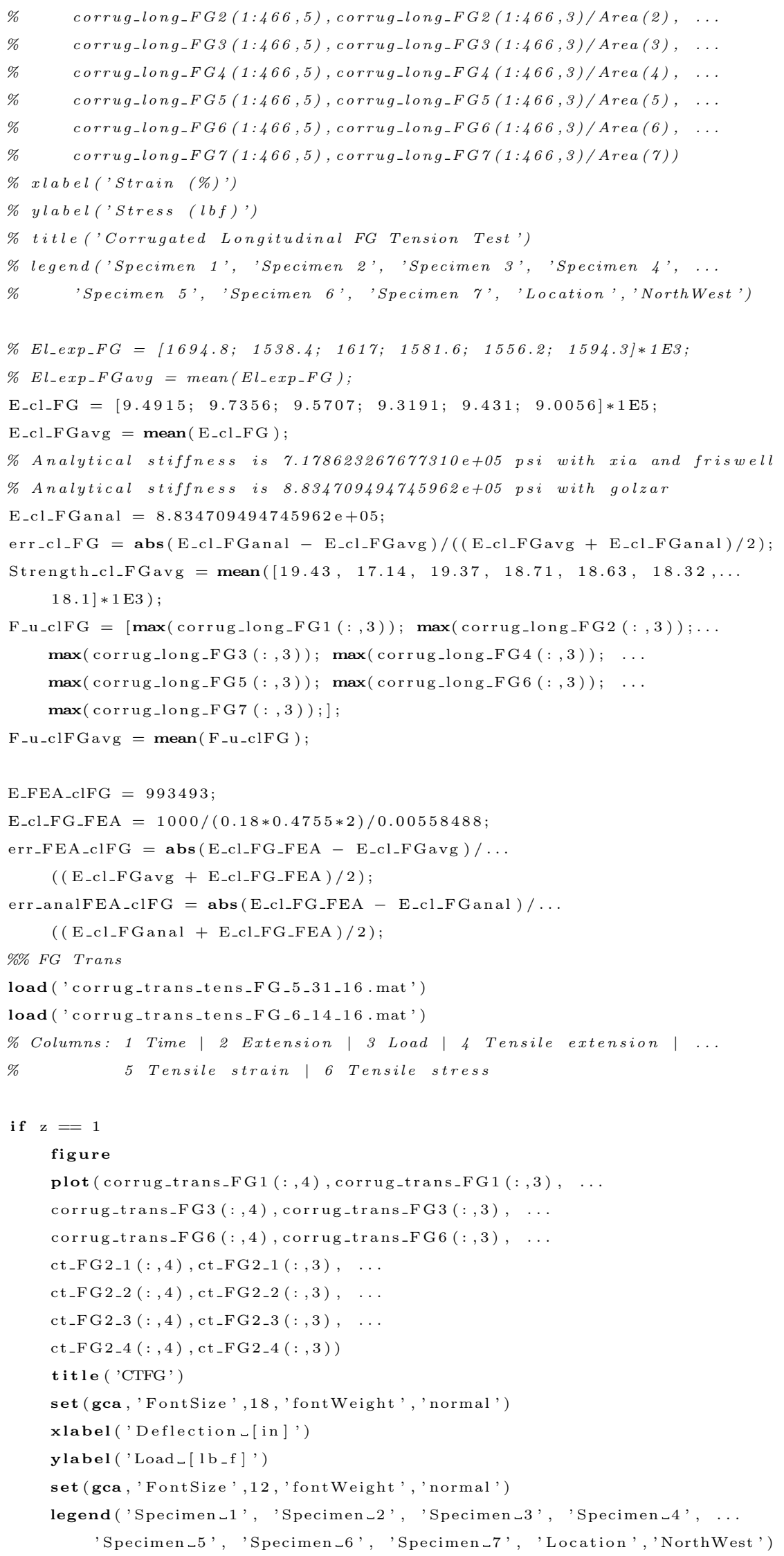


end

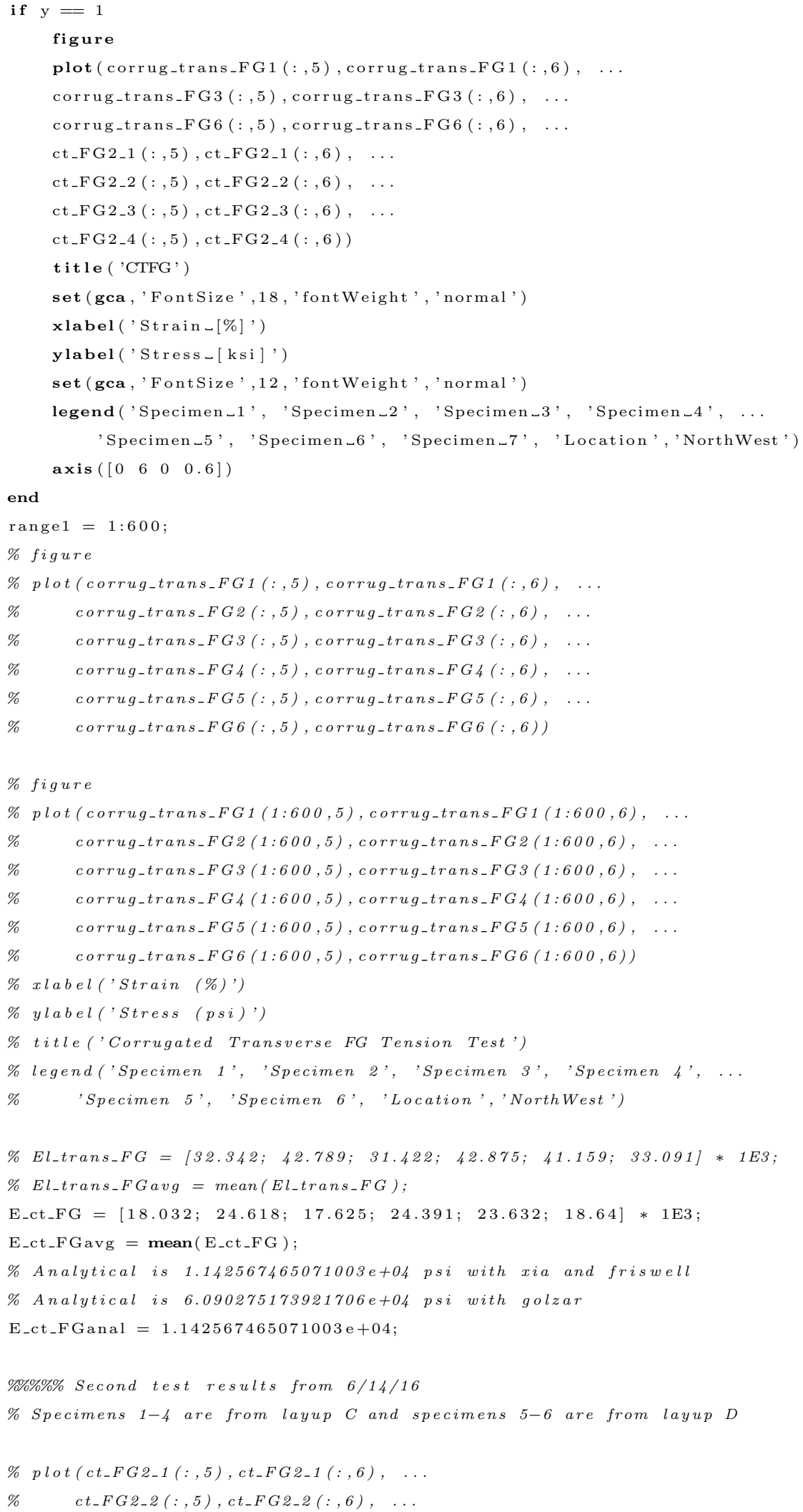




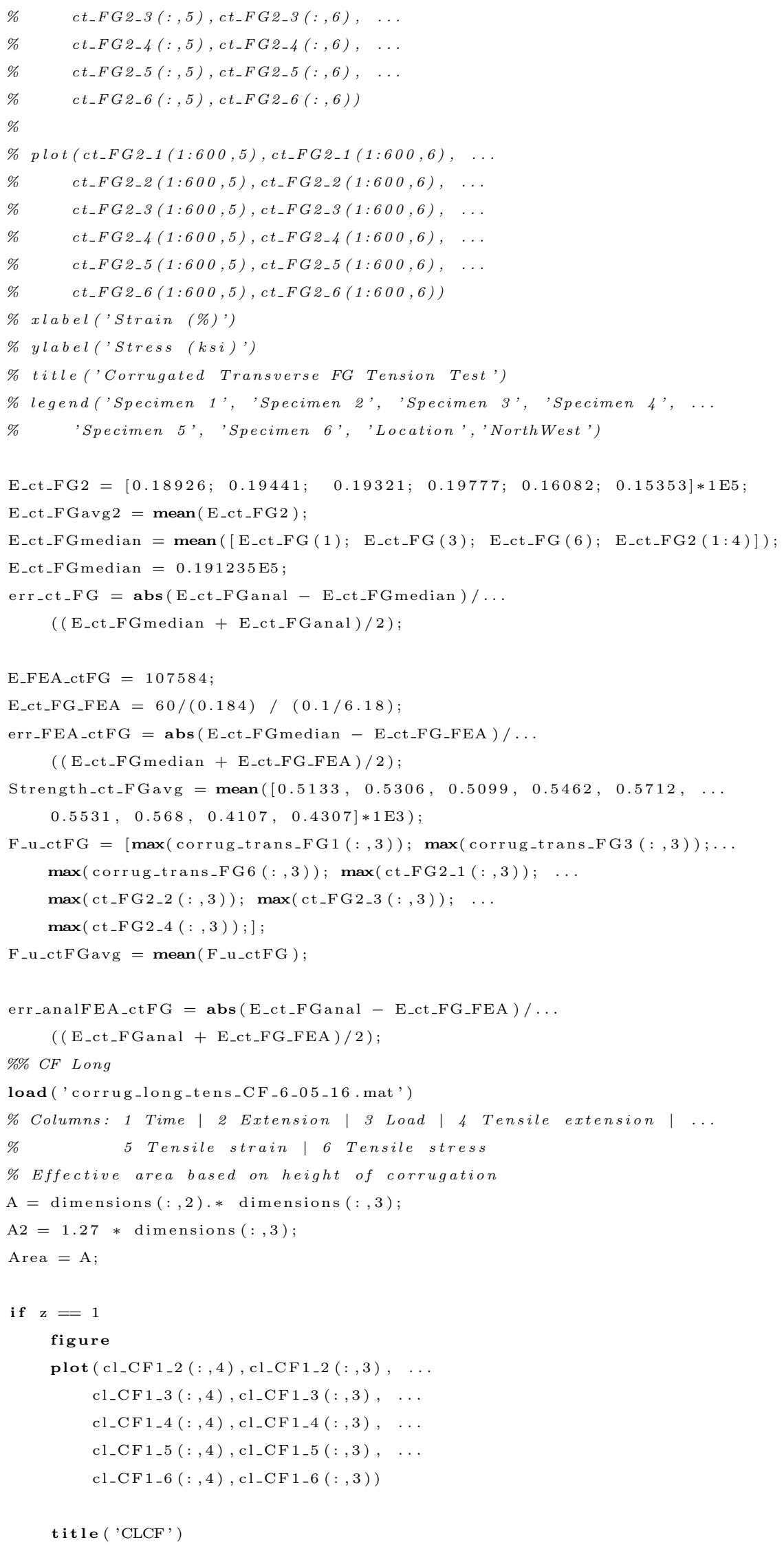




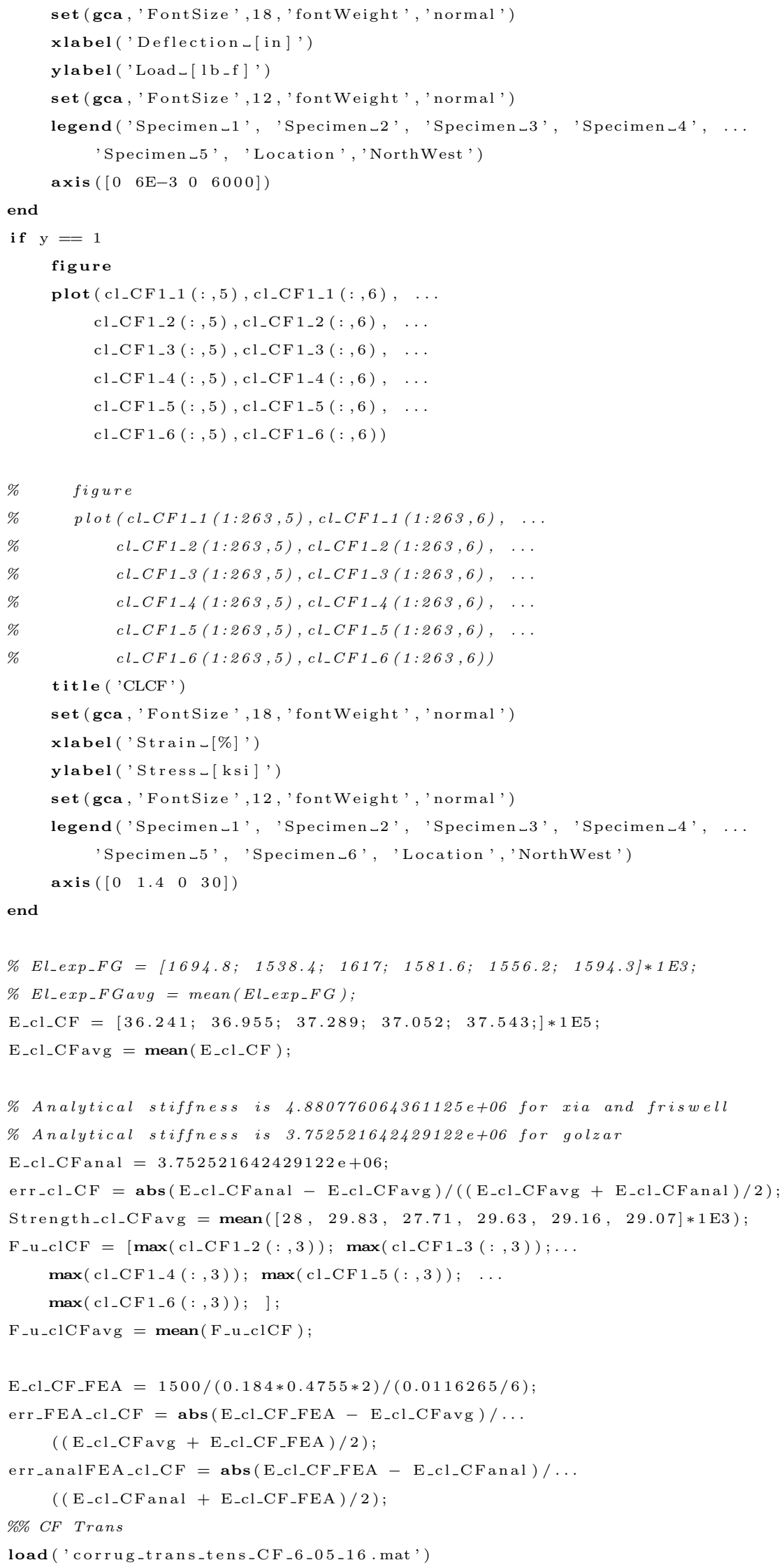




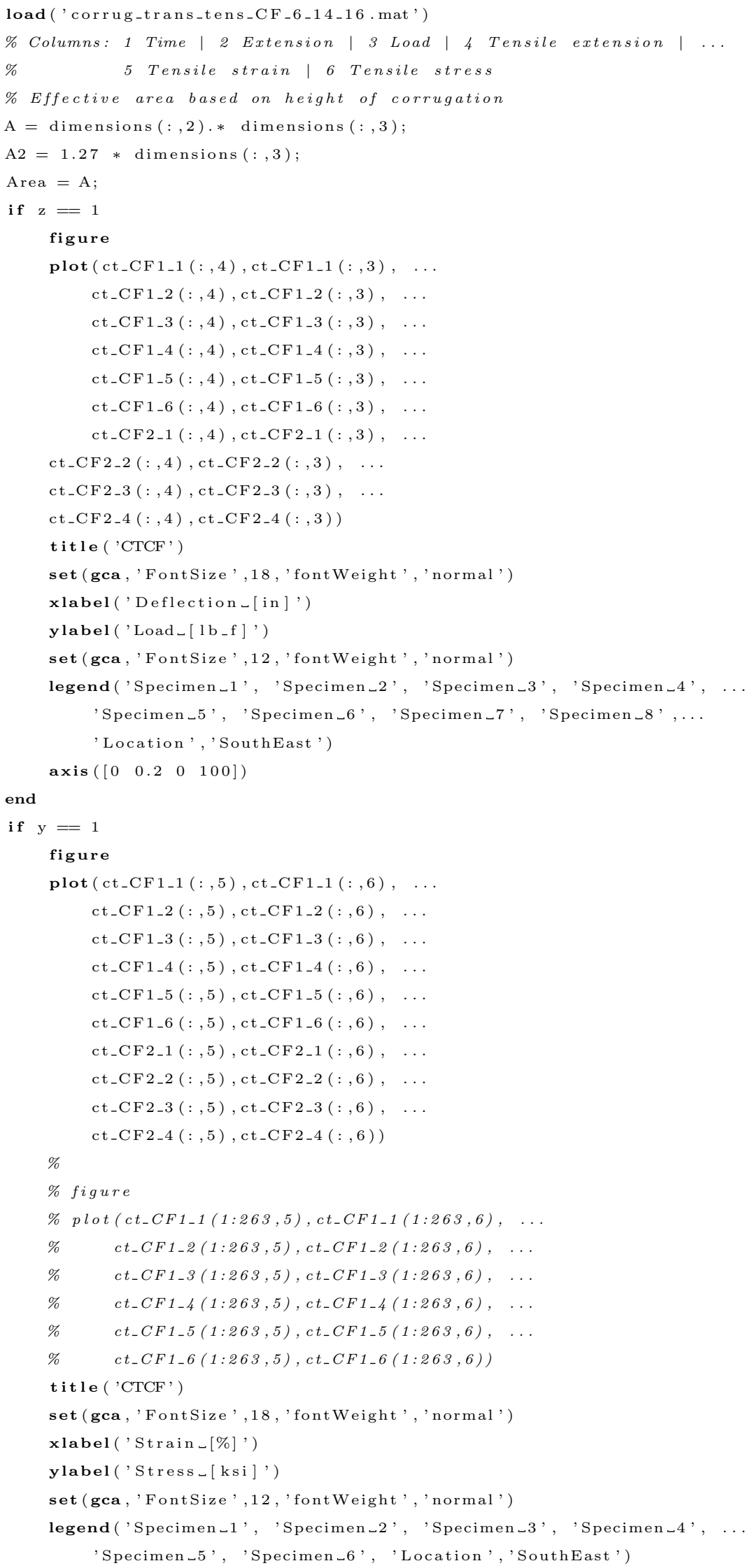




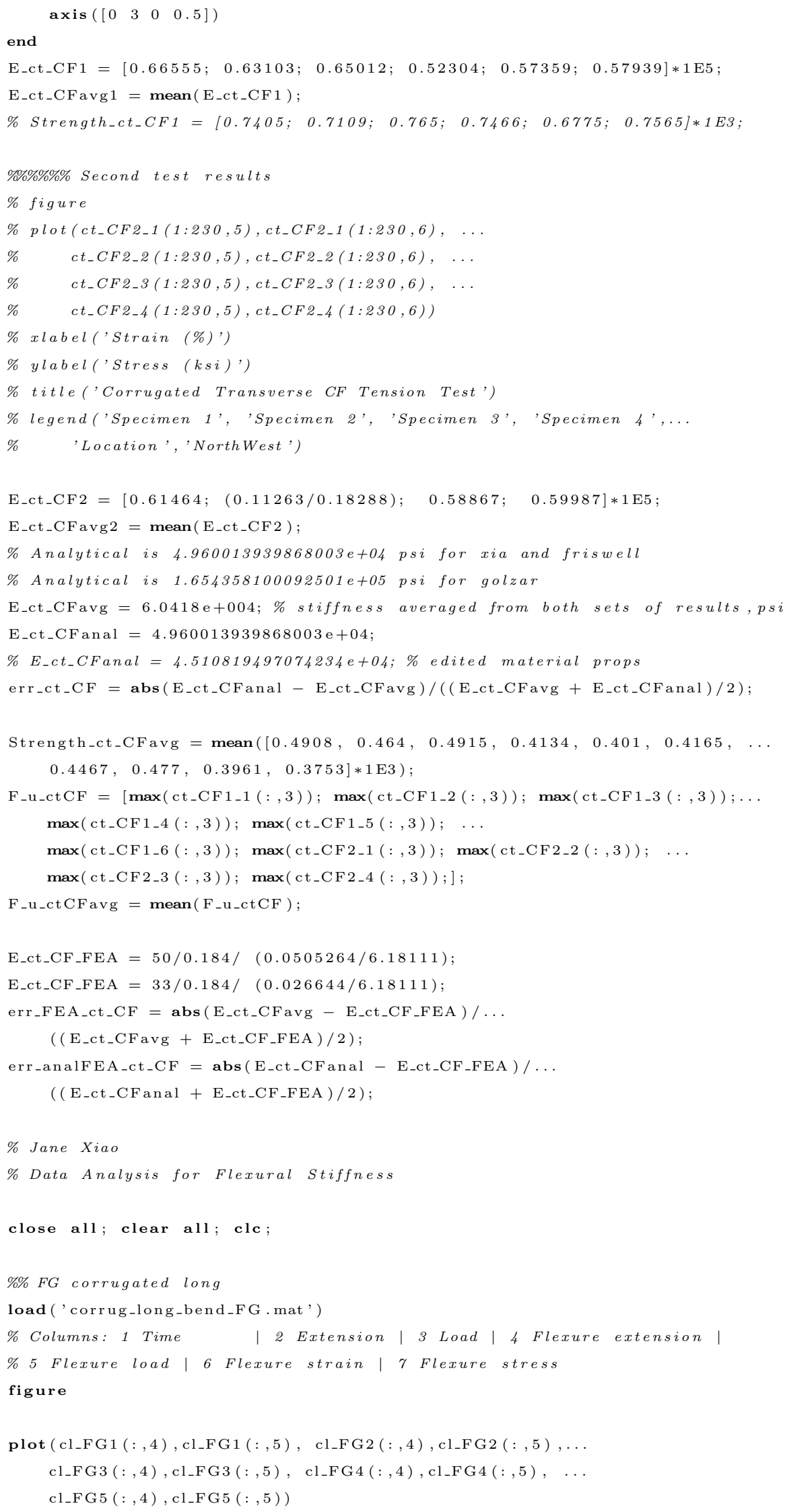




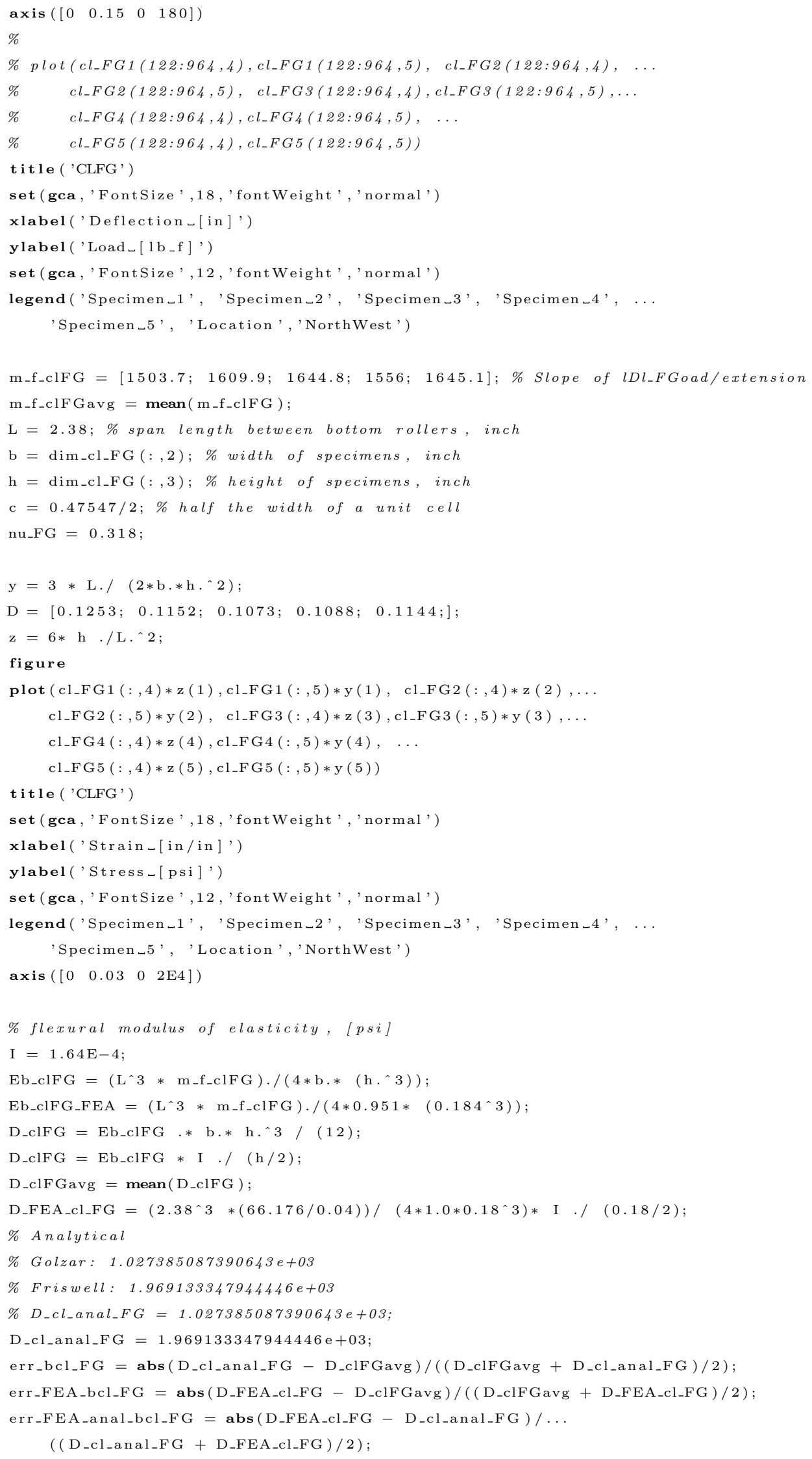




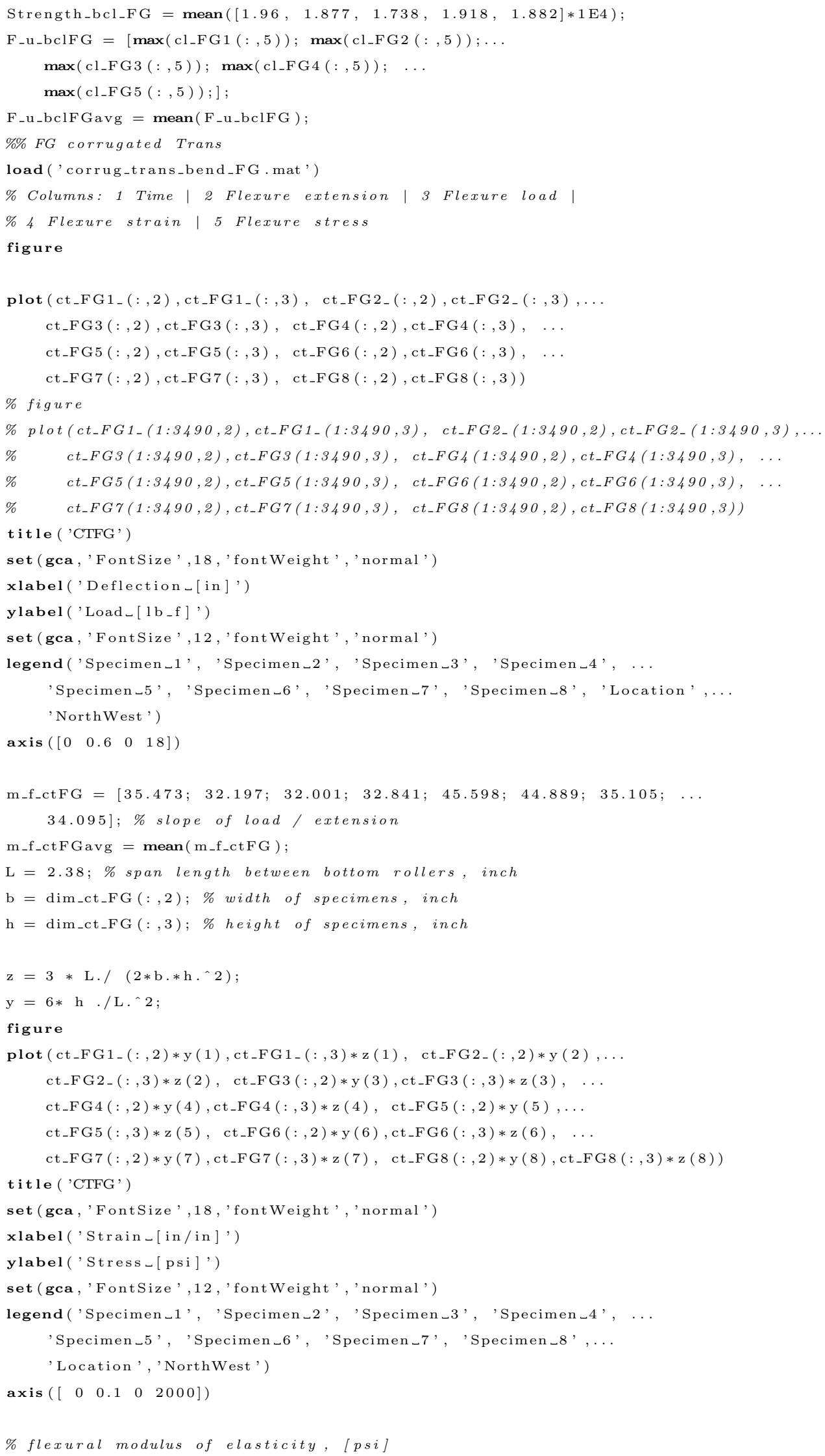




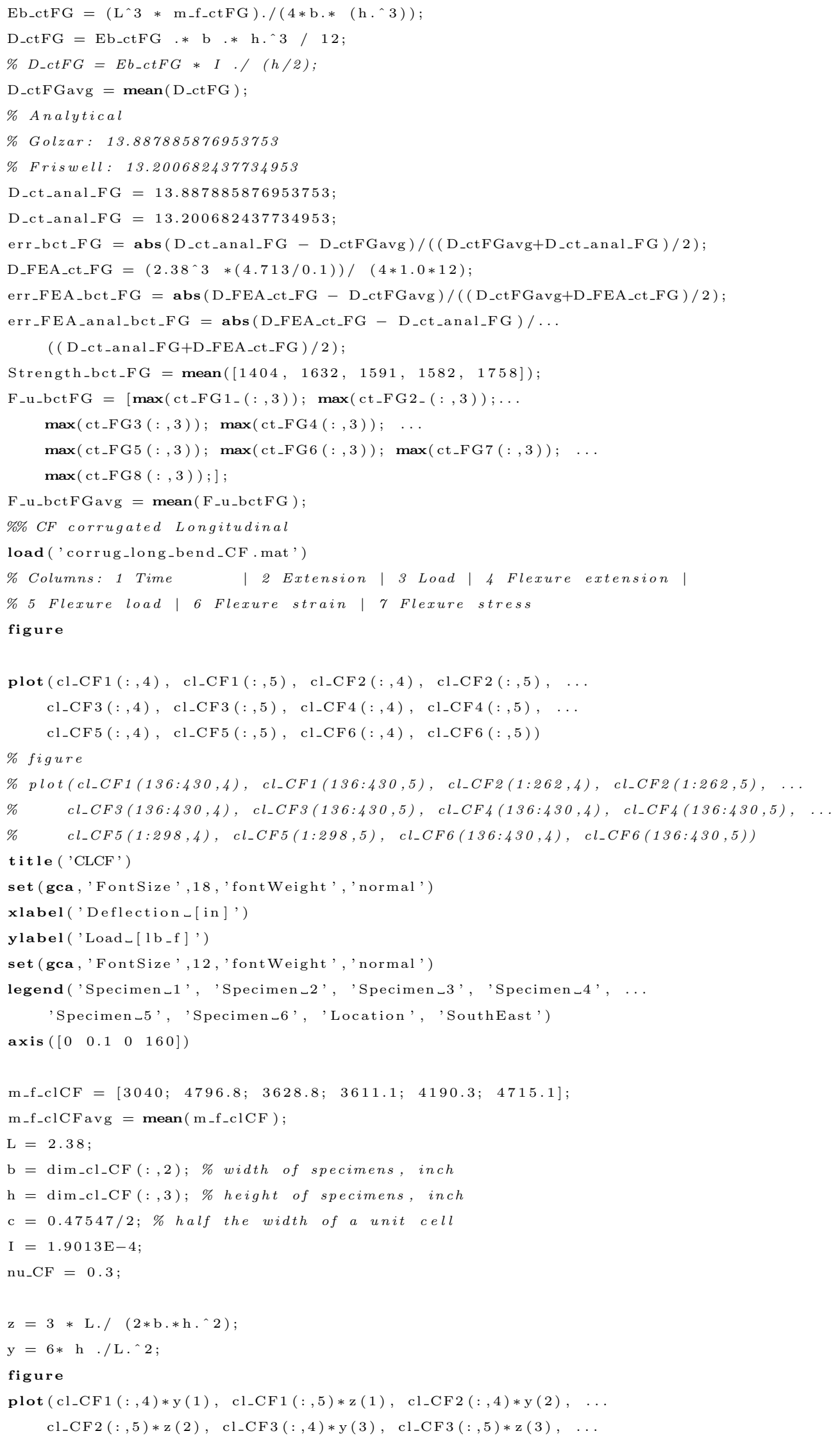




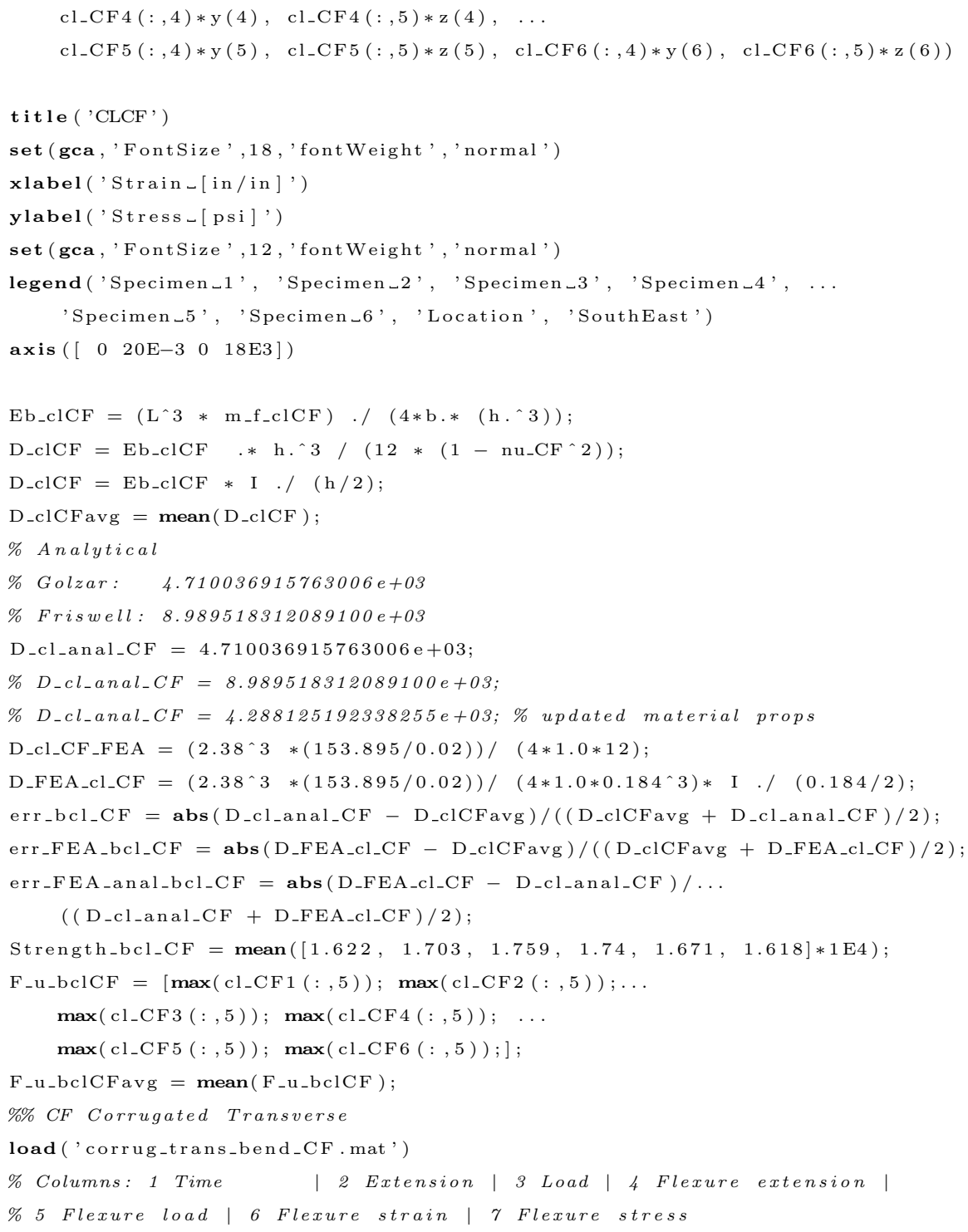




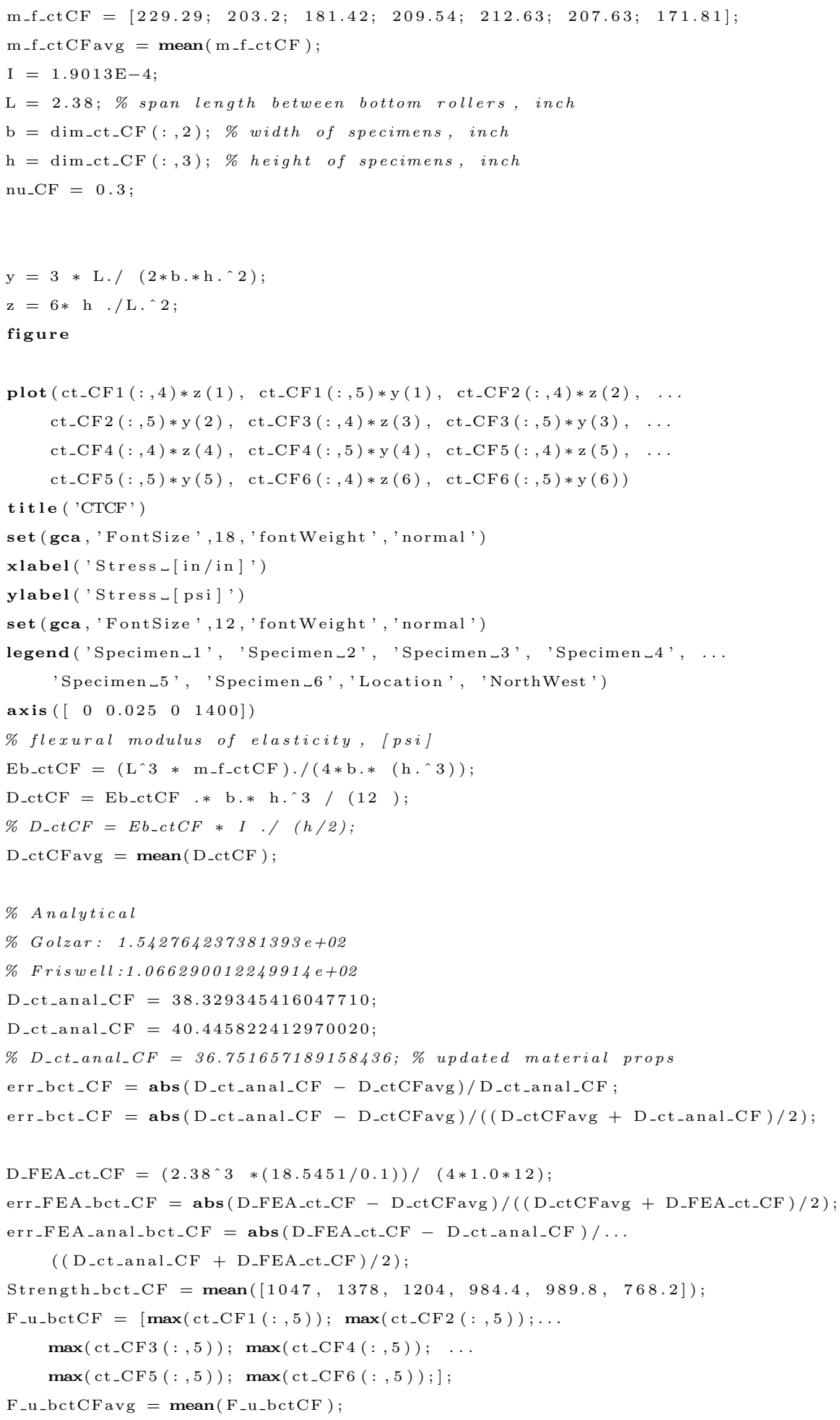

